Data Archived for Events in the Caucasus for the CauSINCollaboration Project

T. Godoladze, D. Hunt, F. Aliyev, A. Arakelyan, D. Kalafat, Z. Javakhishvil, B. Panahi, V. Arzumanyan, L. J. Hutchings, E. Vergino

August 17, 2007 
This document was prepared as an account of work sponsored by an agency of the United States government. Neither the United States government nor Lawrence Livermore National Security, LLC, nor any of their employees makes any warranty, expressed or implied, or assumes any legal liability or responsibility for the accuracy, completeness, or usefulness of any information, apparatus, product, or process disclosed, or represents that its use would not infringe privately owned rights. Reference herein to any specific commercial product, process, or service by trade name, trademark, manufacturer, or otherwise does not necessarily constitute or imply its endorsement, recommendation, or favoring by the United States government or Lawrence Livermore National Security, LLC. The views and opinions of authors expressed herein do not necessarily state or reflect those of the United States government or Lawrence Livermore National Security, LLC, and shall not be used for advertising or product endorsement purposes.

This work performed under the auspices of the U.S. Department of Energy by Lawrence Livermore National Laboratory under Contract DE-AC52-07NA27344. 


\title{
Data Archived for Events in the Caucasus for the CauSIN Collaboration Project
}

\author{
Tea Godoladze ${ }^{1}$, Dina Hunt ${ }^{2 *}$, Fuad Aliyev ${ }^{3}$, Avetis Arakelyan ${ }^{4}$, Dogan Kalafat ${ }^{5}$, Zurab \\ Javakhishvil $^{1}$, Behruz Panahi ${ }^{3}$, Valery Arzumanyan ${ }^{4}$, Lawrence Hutchings ${ }^{2}$, and Eileen Vergino ${ }^{2}$ \\ ${ }^{1}$ Seismic Monitoring Center, Tbilisi Georgia \\ ${ }^{2}$ Lawrence Livermore National Laboratory \\ * currently at Montgomery Watson Harza, Walnut Creek, California \\ ${ }^{3}$ Geology Institute, Azerbaijan National Academy of Sciences, Azerbaijan \\ ${ }^{4}$ Institute of Geological Science of the National Academy of Science of Armenia, Armenia \\ ${ }^{5}$ Kandilli, KOERI, Istanbul, Turkey
}

June 2007

Lawrence Livermore National Lab

The Causin project is a joint effort between the countries in the Caucasus region to develop a Probabilistic Seismic Hazard Analysis. To that end, we have compiled a database of all available network data in the region. The information contained in the database is from four countries: Armenia, Georgia, Azerbaijan, and Turkey and from the Incorporated Research Institutions for Seismology (IRIS). Table 1 lists the networks from which data were obtained. Figure 1 shows the locations of stations, and Figure 2 shows ray paths for event locations of the archived data.

An ongoing effort in the region is the Caucasus Seismic Information Network (CauSIN) is an international scientific project enabling the countries and scientists of the region to: better understand the seismicity of the greater Caucasus; develop new monitoring networks to support the scientific understanding; provide access to seismic data from local networks in the participating countries; and develop a probabilistic seismic hazards assessment for the region enabling the governments in the region to better mitigate the damaging effects from large earthquakes. In parallel with this effort, training has been conducted on modern techniques for probabilistic hazards assessment. CauSIN is also an ongoing effort to promote cooperation in the geosciences between Georgia, Armenia, Azerbaijan, European Union and the United States. The DOE supports the American team from New England Research, Inc. (NER), Massachusetts Institute of Technology (MIT), Boston College, and Lawrence Livermore National Laboratory (LLNL).

\begin{tabular}{|l|l|}
\hline Table 1. Networks used to obtain data & \\
\hline NETWORK & DATES OF EVENTS \\
\hline Georgian-Armenian Strong Motion & $1990-2002$ \\
\hline Georgian Weak Motion & $2003-2005$ \\
\hline Armenian Weak Motion & $1995-2004$ \\
\hline Azerbaijan Analog & $1986-2003$ \\
\hline Azerbaijan Kinemetric & $2004-2006$ \\
\hline Eastern Turkey Seismic Experiment (IRIS) & $1999-2001$ \\
\hline Caucasus Array (IRIS) & $1992-1994$ \\
\hline Historical Waveforms & $1903-1947$ \\
\hline Global Seismograph Network II \& IU(IRIS) & $1990-2004$ \\
\hline
\end{tabular}




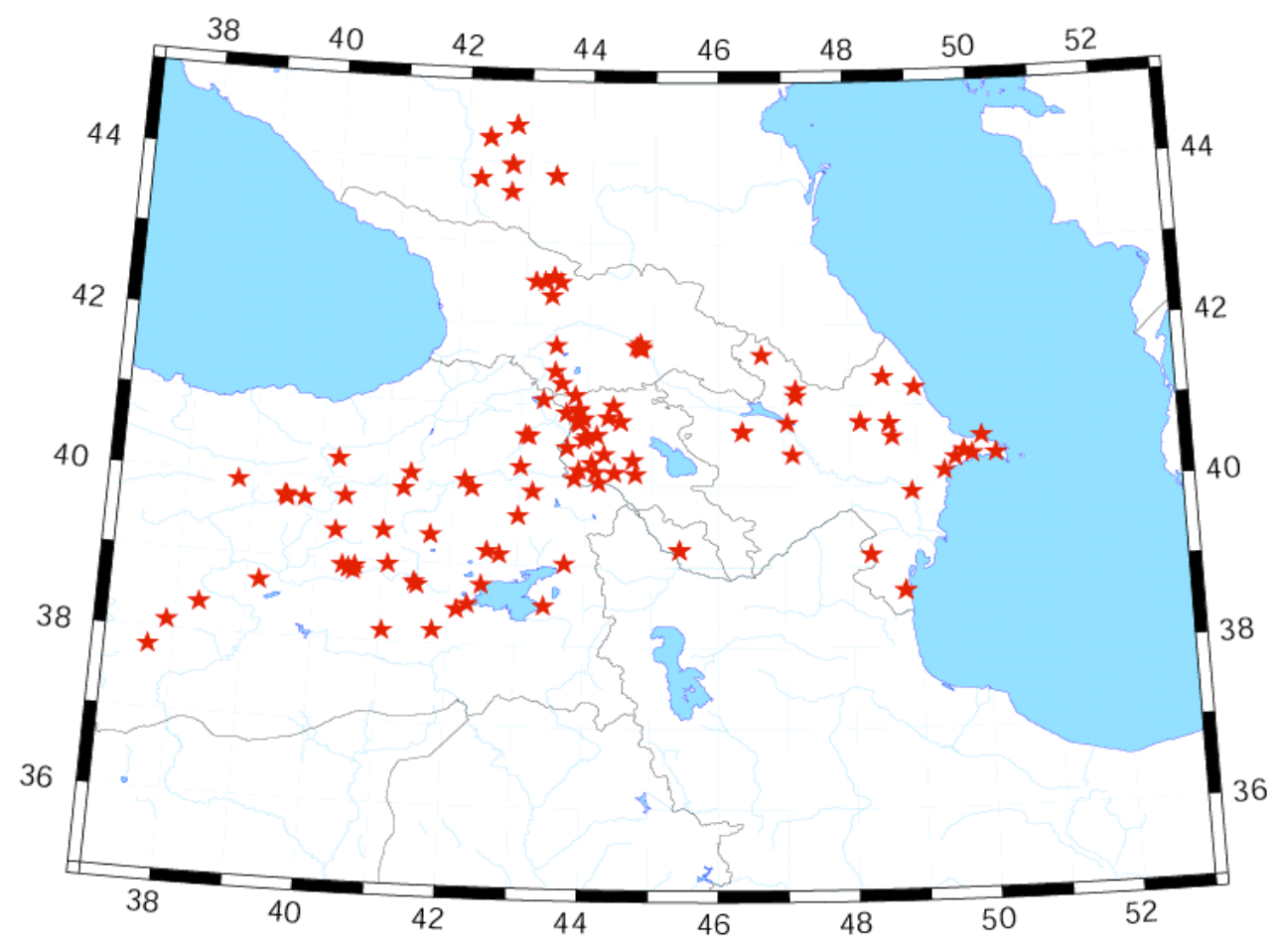

Figure 1. Locations of stations for archived data.

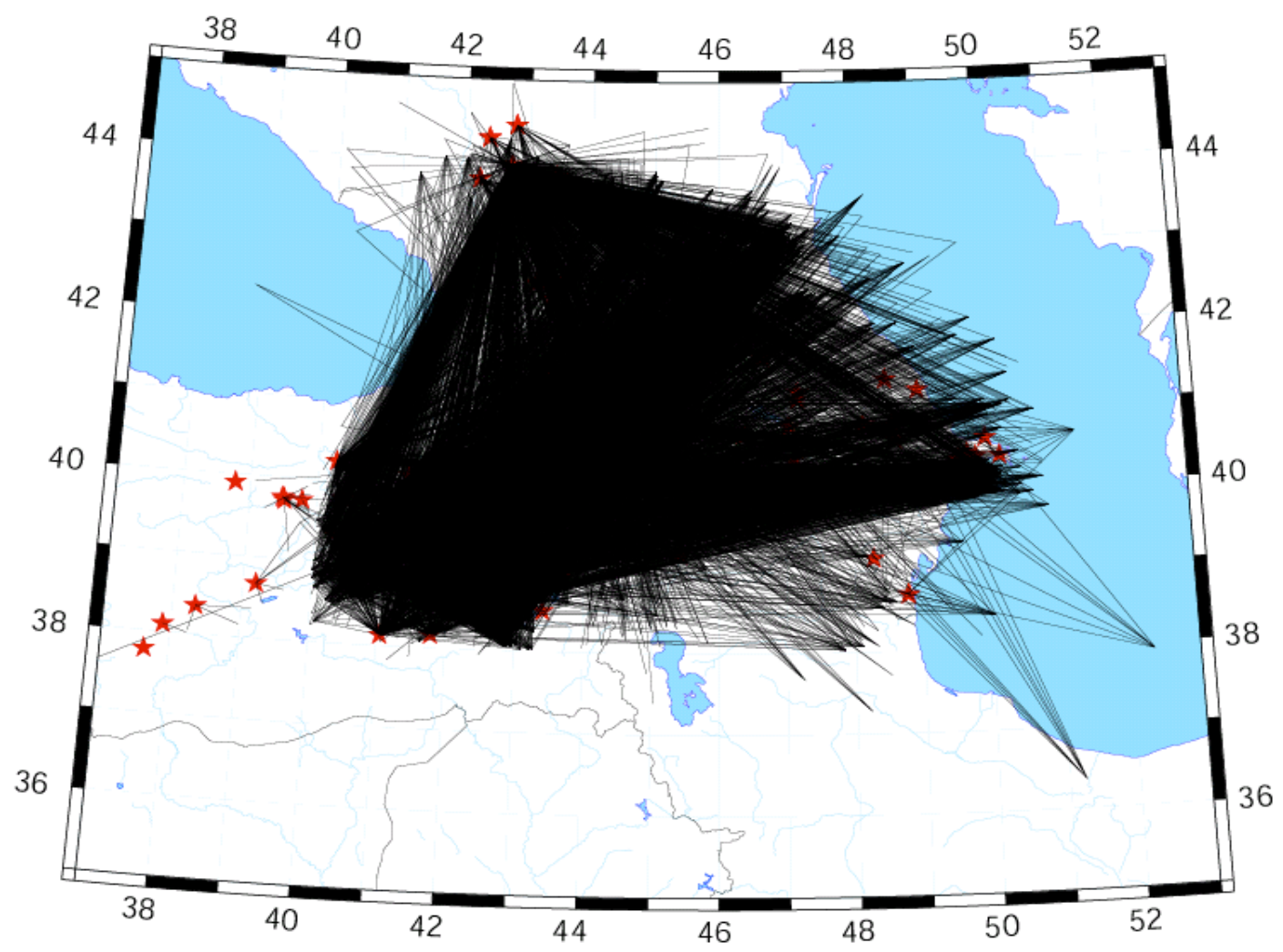

Figure 2. Ray paths for event locations of the archived data. 


\section{- Georgian-Armenian Strong Motion Data}

Georgian-Armenian strong motion data are from 1990-2002 and were supplied by the Seismic Monitoring Center of Georgia. The Georgia-Armenia Strong Motion Network was established by the Swiss Seismological Service. It should be noted that the political situation between the years 1992 and 1995 prevented recordings of most seismic events. Starting in the year 1996, instruments were upgraded and replaced with sufficient back-up batteries in case of power outages.

This network consists of fifteen stations. Nine of the fifteen stations are permanent; three permanent stations are in Armenia, six in Georgia and the other six are portable. There are more than fifteen station locations listed because the portable stations were relocated. Two stations were renamed, Leninakan to Gumri on June 6, 1996 and Kirovakan toVanadzor on February 9, 1997. These data are in SAC format. There are 323 records from this dataset. Coordinates for the events recorded on the portable stations CHU, OBS, SAB and NAD have not been updated in the SAC files. Figure 3 is a plot of all the station locations within the network, Table 2 lists station locations; Figure 4 plots all events from which data were archived.

Instrument response was never obtained for the stations in this network. Some of the same data for these events were obtained from the European Database and Databank for the years 19902005 for the Georgian Region. Some of the information overlaps and there are corrected files available.

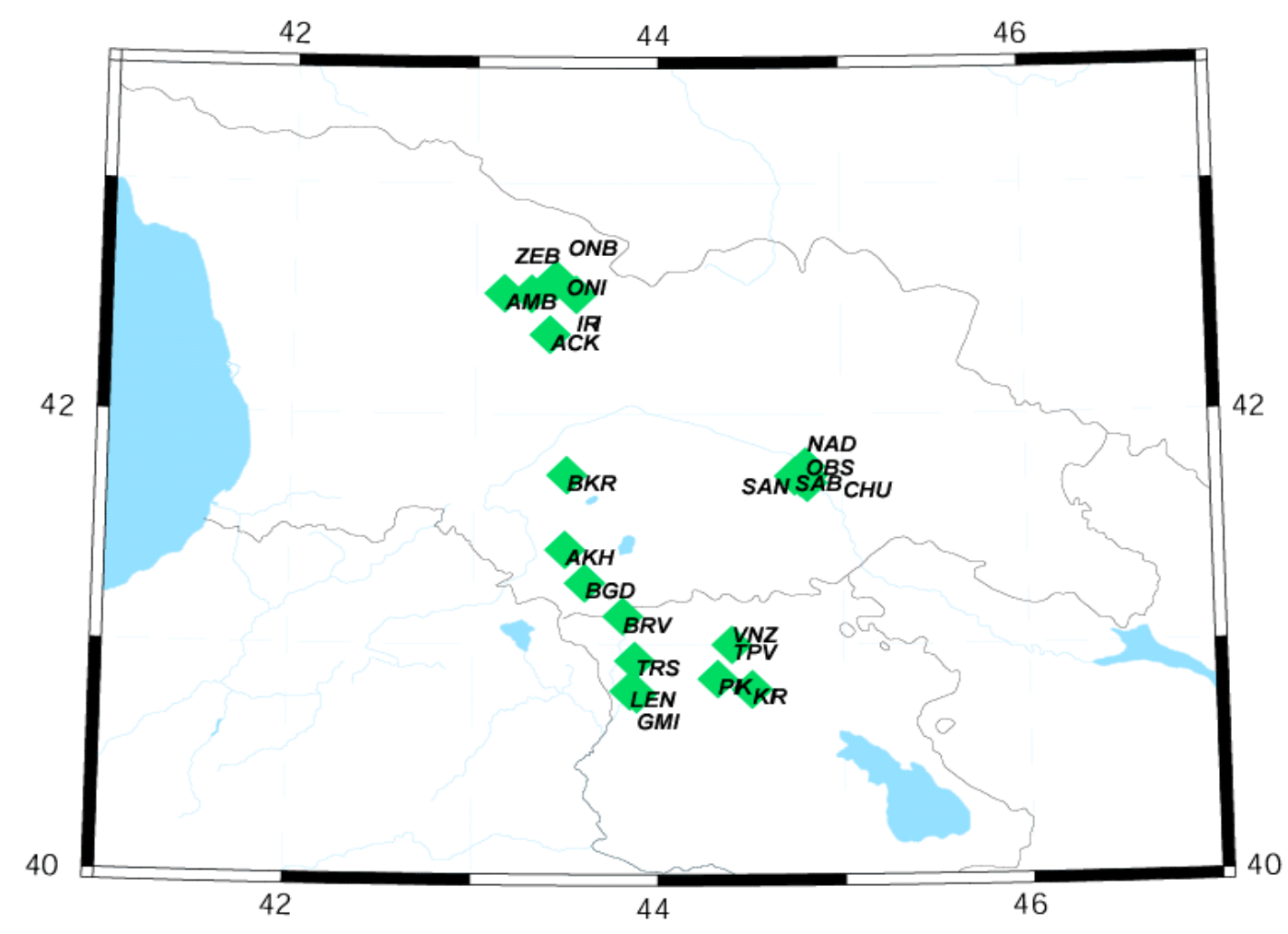

Figure 3. Station locations for the Georgian-Armenian strong motion network. 
Table 2.

\begin{tabular}{|l|l|l|l|l|}
\hline Station & ID & \multicolumn{3}{l|}{ Latitude Longitude Permanent } \\
\hline AKHALKALAKI & SAKH & $41.410 \mathrm{~N}$ & $43.493 \mathrm{E}$ & Yes \\
\hline AMBROLAURI & SAMB & $42.522 \mathrm{~N}$ & $43.155 \mathrm{E}$ & No \\
\hline BAKURIANI & SBKR & $41.734 \mathrm{~N}$ & $43.502 \mathrm{E}$ & Yes \\
\hline BAVRA & SBVR & $41.120 \mathrm{~N}$ & $43.809 \mathrm{E}$ & Yes \\
\hline BOGDANOVKA & SBGD & $41.265 \mathrm{~N}$ & $43.600 \mathrm{E}$ & Yes \\
\hline CHUGURETI & SCHU & $41.700 \mathrm{~N}$ & $44.810 \mathrm{E}$ & No \\
\hline ERZINCAN-AIRPORT & SERA & $39.717 \mathrm{~N}$ & $39.517 \mathrm{E}$ & No \\
\hline ERZINCAN-EKSISU & SERE & $39.733 \mathrm{~N}$ & $39.783 \mathrm{E}$ & No \\
\hline ERZINCAN-METRO & SERM & $39.752 \mathrm{~N}$ & $39.489 \mathrm{E}$ & No \\
\hline GUMRI & SGMI & $40.783 \mathrm{~N}$ & $43.883 \mathrm{E}$ & Yes \\
\hline IRI & SIRI & $42.517 \mathrm{~N}$ & $43.551 \mathrm{E}$ & No \\
\hline KIRVOKAN & SKIR & $40.804 \mathrm{~N}$ & $44.506 \mathrm{E}$ & Yes \\
\hline LENINAKAN & SLEN & $40.796 \mathrm{~N}$ & $43.847 \mathrm{E}$ & Yes \\
\hline NADZALADEVI & SNAD & $41.730 \mathrm{~N}$ & $44.810 \mathrm{E}$ & No \\
\hline OBSERVATORY & SOBS & $41.770 \mathrm{~N}$ & $44.800 \mathrm{E}$ & No \\
\hline ONI & SONI & $42.583 \mathrm{~N}$ & $43.440 \mathrm{E}$ & No \\
\hline ONI-BASE_CAMP & SONB & $42.573 \mathrm{~N}$ & $43.436 \mathrm{E}$ & No \\
\hline SABURTALO & SSAB & $41.730 \mathrm{~N}$ & $44.740 \mathrm{E}$ & No \\
\hline SACXERE & SACK & $42.345 \mathrm{~N}$ & $43.407 \mathrm{E}$ & No \\
\hline SANZONA & SSAN & $41.720 \mathrm{~N}$ & $44.780 \mathrm{E}$ & No \\
\hline SPITAK-KARADZOR & SPIK & $40.848 \mathrm{~N}$ & $44.320 \mathrm{E}$ & Yes \\
\hline STEPANAVAN & STPV & $40.997 \mathrm{~N}$ & $44.396 \mathrm{E}$ & Yes \\
\hline TOROS & STRS & $40.928 \mathrm{~N}$ & $43.873 \mathrm{E}$ & Yes \\
\hline VANDZOR & SVNZ & $40.804 \mathrm{~N}$ & $44.506 \mathrm{E}$ & Yes \\
\hline ZEMO_BARI & SZEB & $42.522 \mathrm{~N}$ & $43.305 \mathrm{E}$ & No \\
\hline
\end{tabular}

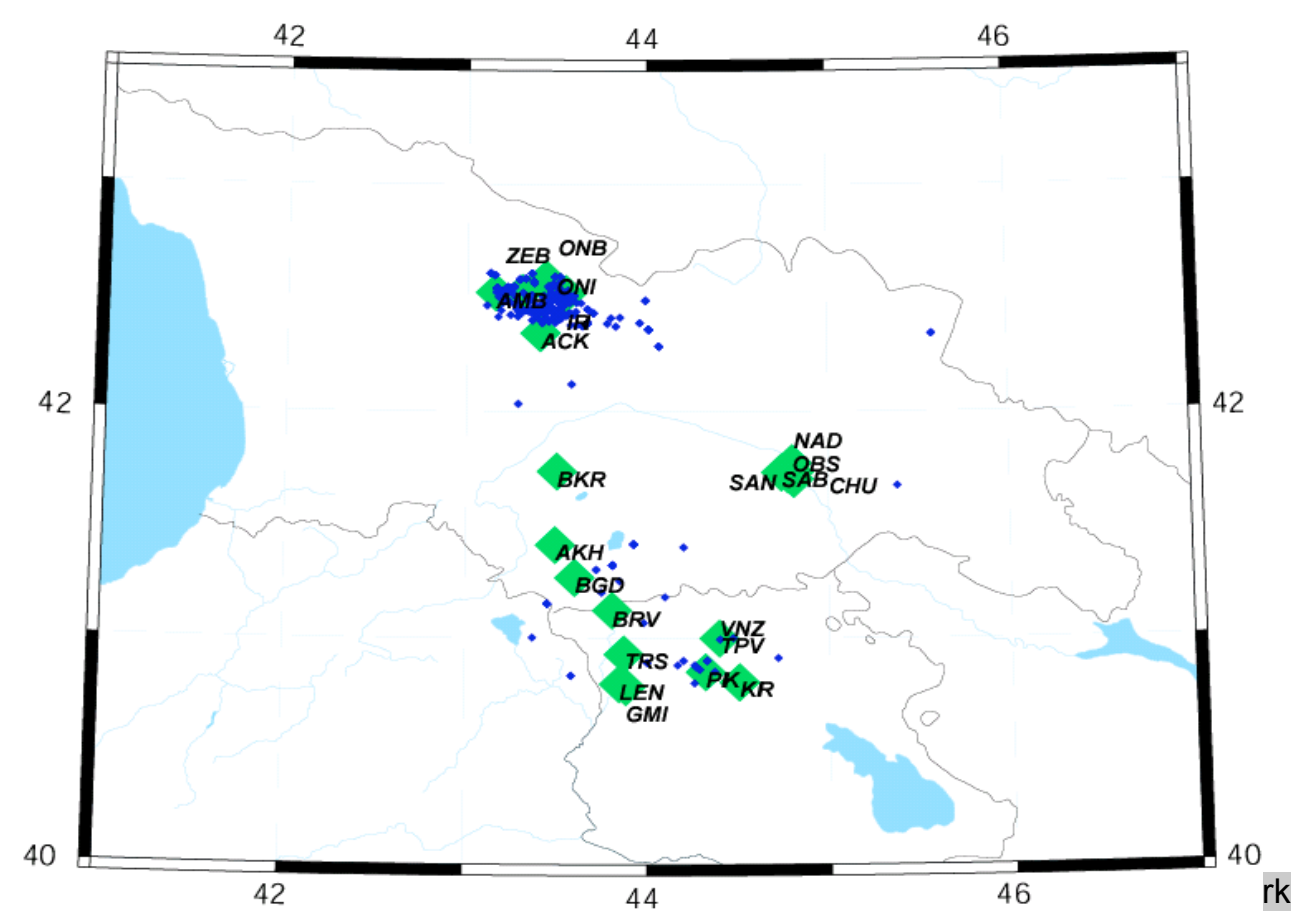

Figure 4. Events for which data were archived. 


\section{- Georgian Weak Motion Data}

Figure 5 shows station locations the Georgian Weak Motion Network. This network consists of 5 stations, and data are available for the years 2003-2005. These data were obtained from the Seismic Monitoring Center of Georgia along with the instrument response. These data were entered into the database, but because a catalog for the years 2003-2005 has not been obtained the format of the files represents the start time of the machine and not the origin time of the earthquake.

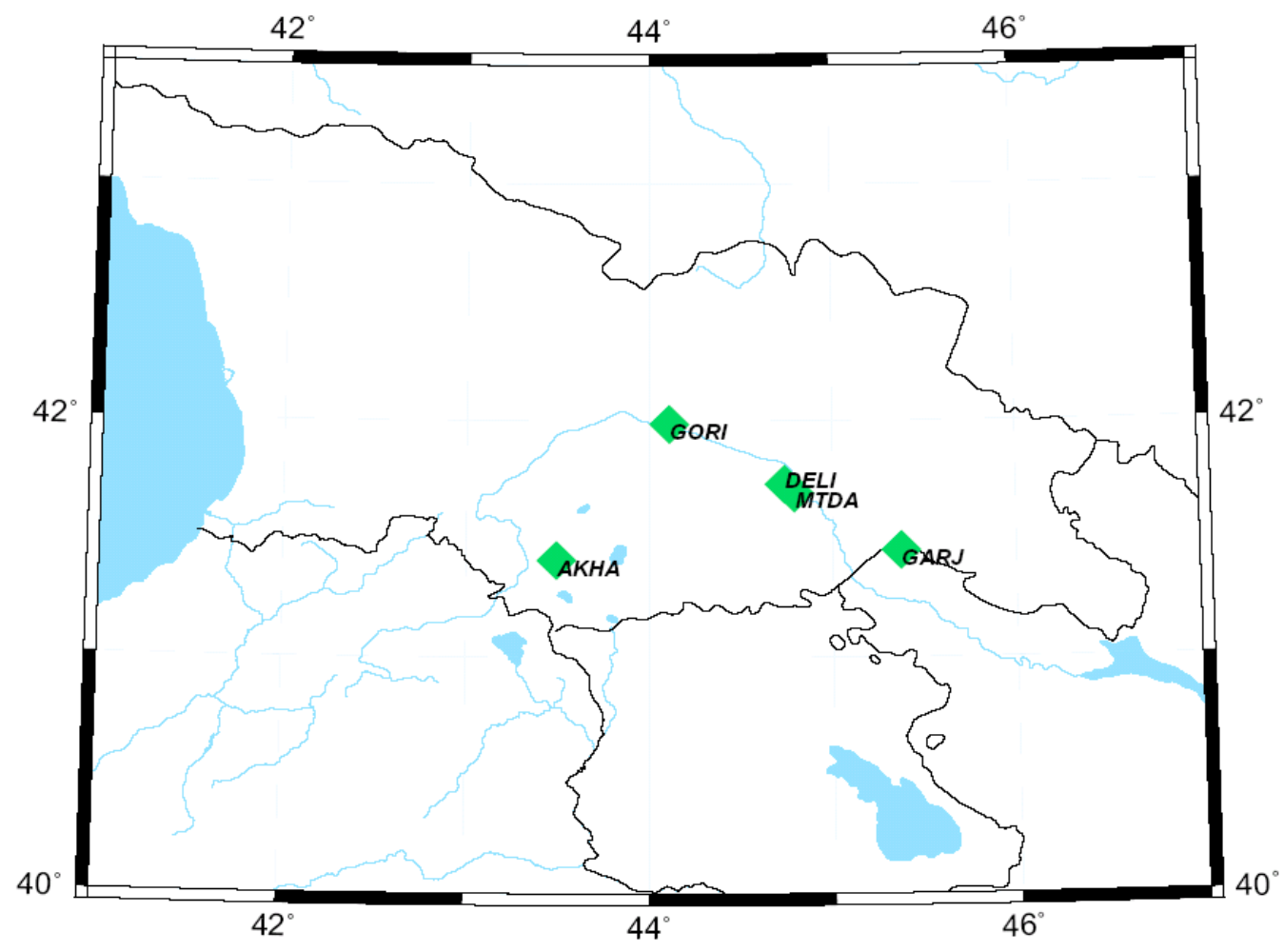

Figure 5. Station locations the Georgian Weak Motion Network

Table 3. Station locations.

\begin{tabular}{|l|ll|l|} 
Station & ID & Latitude & \multicolumn{1}{c|}{ Longitude } \\
\hline AKHALKALAKI & AKHA & $41.69393 \mathrm{~N}$ & $43.49279 \mathrm{E}$ \\
\hline & DELI & $41.73092 \mathrm{~N}$ & $44.73819 \mathrm{E}$ \\
\hline & GARJ & $41.45071 \mathrm{~N}$ & $45.37317 \mathrm{E}$ \\
\hline & GORI & $41.98551 \mathrm{~N}$ & $44.10877 \mathrm{E}$ \\
\hline & MTDA & $41.69393 \mathrm{~N}$ & $44.79252 \mathrm{E}$ \\
\hline
\end{tabular}




\section{- Armenian Weak Motion Data}

The Armenian weak motion data were supplied by the National Survey for Seismic Protection. These records are from the years 1995-2002. Included with these records are IRIS data from one station (GUM) for the years 1997, and 1999-2002. All the data were received in plain text or ASCII and then converted to SAC. A total of 17 stations, recorded a total of 217 events. Some inconsistencies exist in the number of stations that recorded specific events; either the dataset is not complete or there were equipment failures. Another problem that needs to be addressed is that some of the records contain timing errors; i.e., some stations record the earthquake before its cataloged time. Figure 6 is a plot of the Armenian weak motion network. It can be seen from the plot that the network is concentrated in the northwestern part of Armenia. Table 4 lists station locations. Figure 7 plots events archived from the network.

New data have been obtained for the years 2003-2005. The files have been added to the database and will be updated as soon as catalog information is received.

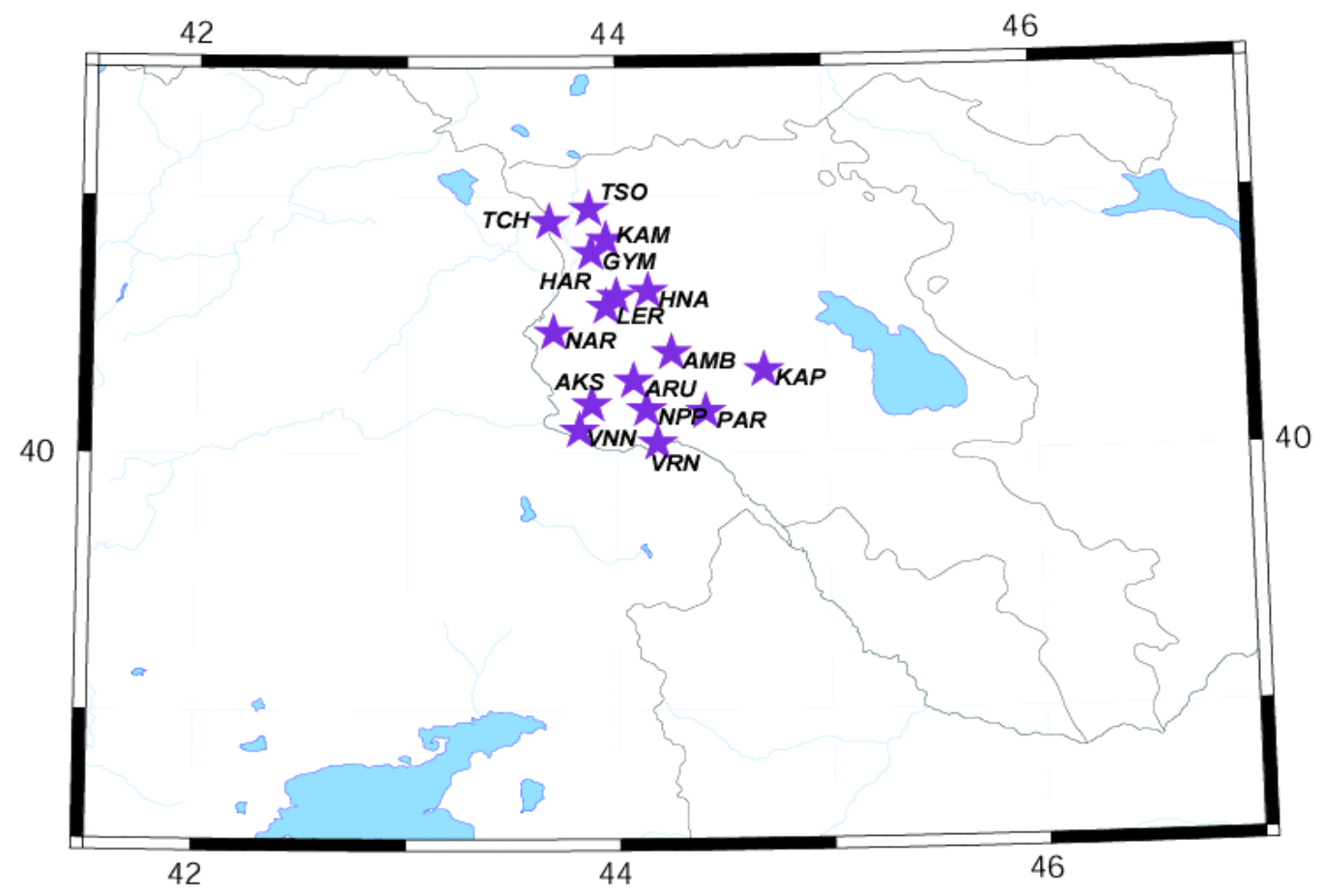

Figure 6. Armenian weak motion network 
Table 4. Station locations.

\begin{tabular}{|c|c|c|c|}
\hline Station & ID & Latitude & Longitude \\
\hline AMBERD & AMB & 40.39 & 44.26 \\
\hline ARAKS & AKS & 40.19 & 43.88 \\
\hline ARUCH & ARU & 40.28 & 44.08 \\
\hline GYUMRI & GYM & 40.78 & 43.88 \\
\hline HARICH & HAR & 40.61 & 44 \\
\hline HNABERD & HNA & 40.63 & 44.15 \\
\hline KAMO & KAM & 40.83 & 43.95 \\
\hline KAPUTAN & KAP & 40.32 & 44.7 \\
\hline LERNAKERT & LER & 40.57 & 43.95 \\
\hline METSAMOR & NPP & 40.17 & 44.14 \\
\hline NOR ARTIK & NAR & 40.47 & 43.7 \\
\hline PARAKAR & PAR & 40.16 & 44.42 \\
\hline TCHAKHMAKH & $\mathrm{TCH}$ & 40.9 & 43.68 \\
\hline TSOGHAMARG & TSO & 40.95 & 43.87 \\
\hline VANAND & VNN & 40.09 & 43.82 \\
\hline VARDANASHEN & $\begin{array}{l}\mathrm{V} \\
\mathrm{V} R \mathrm{RN}\end{array}$ & 40.04 & 44.19 \\
\hline
\end{tabular}

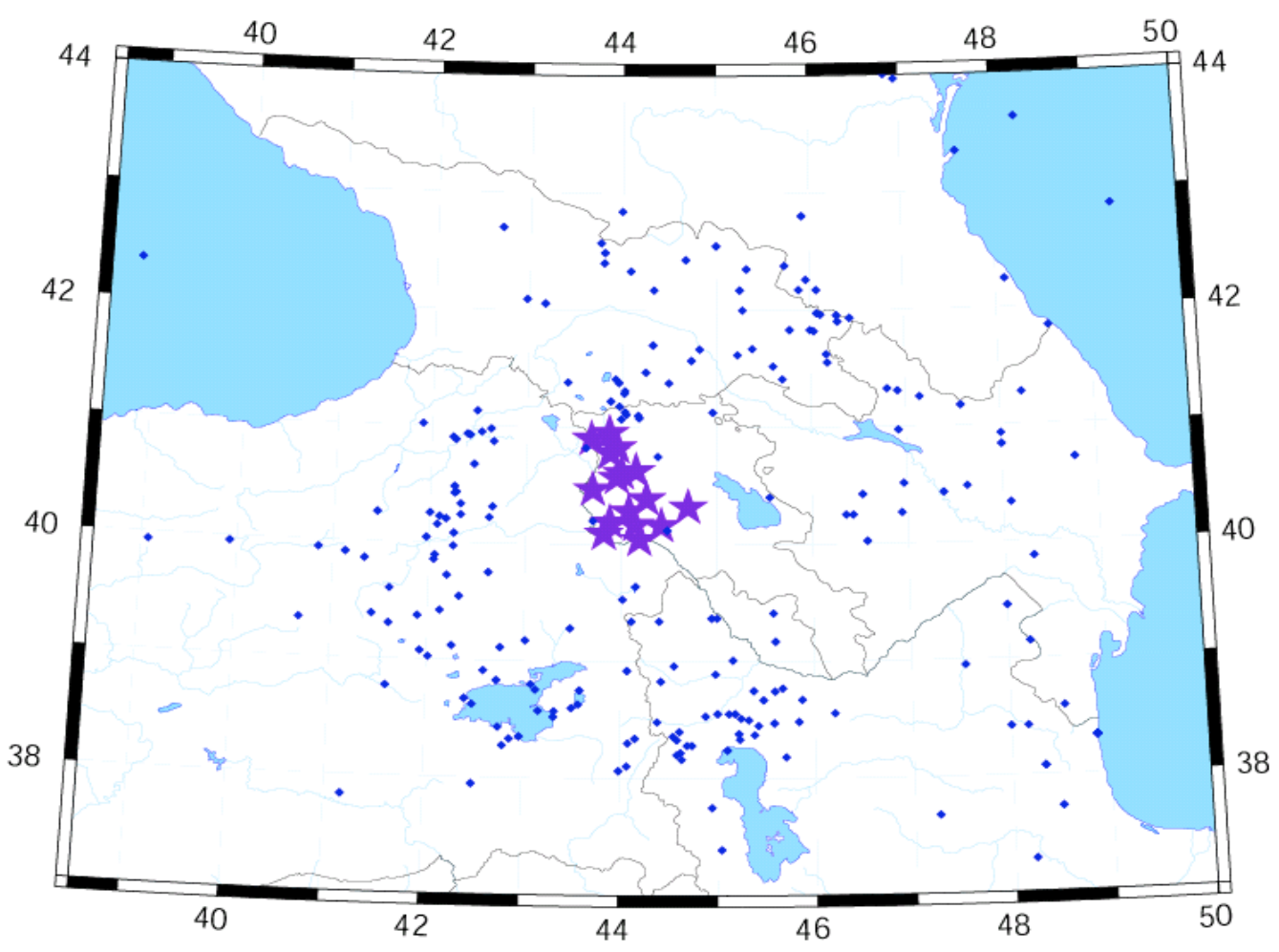

Figure 7. Events archived from the network. 


\section{- Azerbaijan Analog Network}

The Azerbaijan analog network was originally the Unified Soviet Network. The Azeri data were supplied in a Microsoft ACCESS database that contained files in EXCEL spreadsheets. The data span the years 1986-2003. Overall, seven stations recorded twelve events for a total of twenty waveform records; each station did not record every event. This dataset has been converted to SAC format. Some inconsistencies exist in the information, i.e. stations' recording only one component or the event is not found in the catalog of the entire Caucasus. For certain earthquakes there is no information in the catalog because they were recorded in 2003 and the catalog only extends to 2002. Catalog information was found in the ACCESS database for the rest of the events; however the epicenter coordinates are missing for a few files. The Azerbaijanians are in the process of supplying the catalog information for the year 2003. The information that was available was put in the header of each SAC file. Although the time representation has no mark of reference, it is known that the time was derived from old type seismograms and the units were relatively separated in an automatic manner. The time the instrument was triggered was available in the database, but this did not correspond to the time for the first record (plot in excel contained time vs. amplitude). It was assumed that the beginning number in the excel file was the begin time of the rupture in reference to GMT time. Figure 8 is a plot of the stations, and events for which data are archived. The pink circles denote stations in the network, black triangles represent that there are waveform data for this station, and blue diamonds are event locations. Table 5 lists station locations.

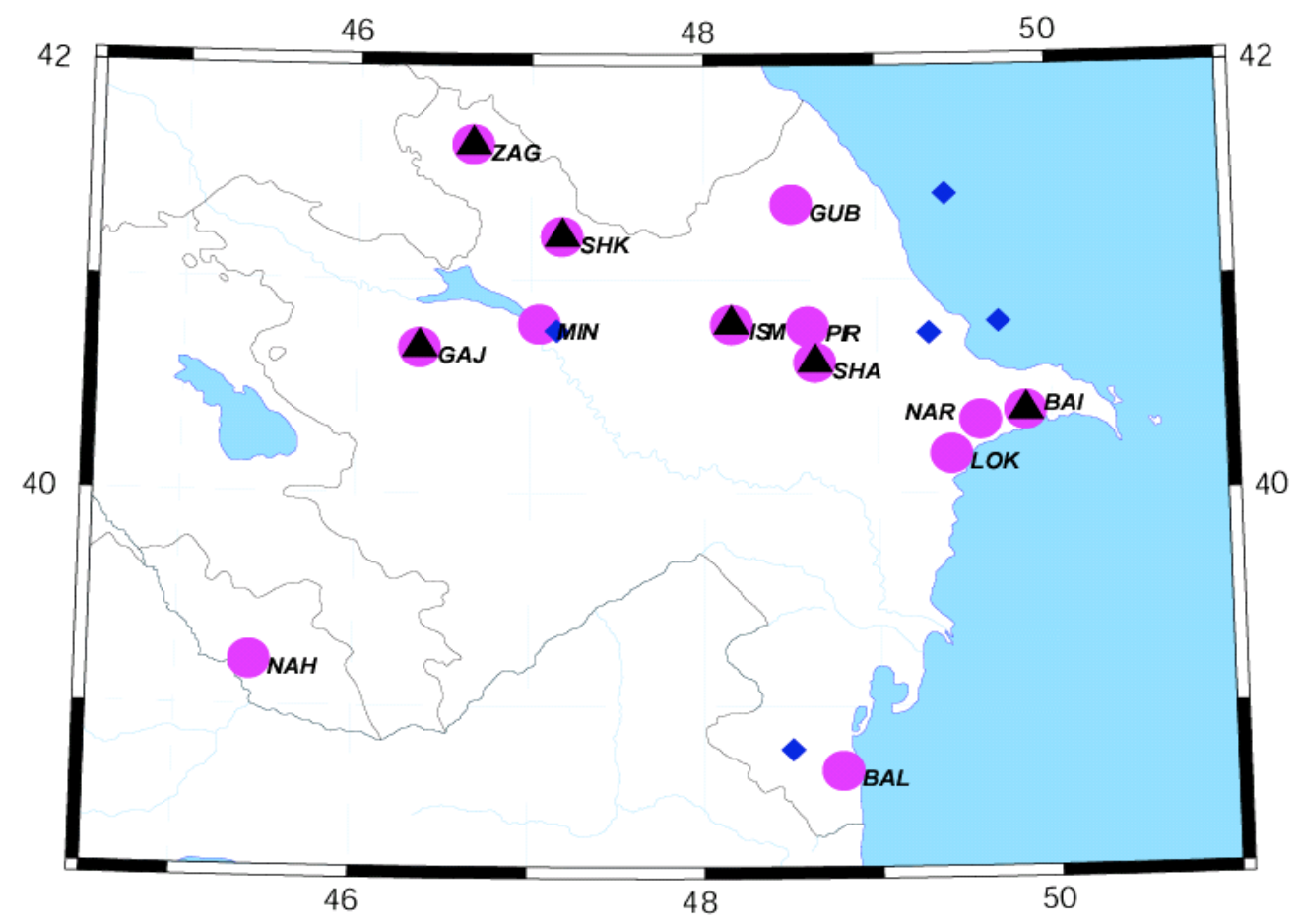
$\boldsymbol{A}_{\text {Stations with Records }}$
Stations in the Network

Figure 8. Stations and events of the Azerbaijan Analog Network. 
Table 5. Station locations

\begin{tabular}{|l|ll|l|}
\hline \multicolumn{4}{|c|}{ Azerbaijan Analog Stations } \\
\hline \multicolumn{1}{|c|}{ Station } & \multicolumn{1}{c|}{ ID* } & \multicolumn{1}{c|}{ Latitude Longitude } \\
\hline BAKU & BAI & 40.38 & 49.85 \\
\hline GUBA & GUB & 41.35 & 48.51 \\
\hline ISMAILLI & ISM & 40.79 & 48.16 \\
\hline MINGECHEV & MIN & 40.79 & 47.05 \\
\hline SHEKI & SHK & 41.2 & 47.18 \\
\hline ZAGATALA & ZAG & 41.63 & 46.66 \\
\hline GYANDJA & GAJ & 40.68 & 46.36 \\
\hline NAHCHIVAN & NAH & 39.21 & 45.42 \\
\hline SHAMAKHI & SHA & 40.61 & 48.64 \\
\hline PIRGULU & PIR & 40.78 & 48.6 \\
\hline BALLABUR & BAL & 38.7 & 48.78 \\
\hline CHILOV I. & CHI & 40.19 & 50.35 \\
\hline LOK-BATAN & LOK & 40.18 & 49.42 \\
\hline NARDARAN & NAR & 40.34 & 49.59 \\
\hline AGDAM & AGD & 40.10 & 47.00 \\
\hline KELBEJAR & KAL & 40.10 & 46.05 \\
\hline JEBRAIL & JEB & 39.40 & 47.02 \\
\hline
\end{tabular}

*Station ID's created, none exist.

\section{- Azerbaijan Kinemetrics Network}

The Azerbaijan 14 station, Kinemetrics network is co-located with the Azerbaijan analog network stations, and have the same identification (Table 6). Data obtained span four months: March 27, 2004 through July 3, 2004. After converting these data to SAC format it was discovered that longer, more complete records would be needed. These longer waveforms are in the process of being submitted along with any new recordings. Figure 9 plots station locations

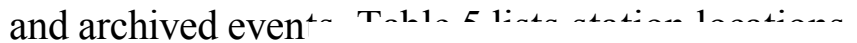

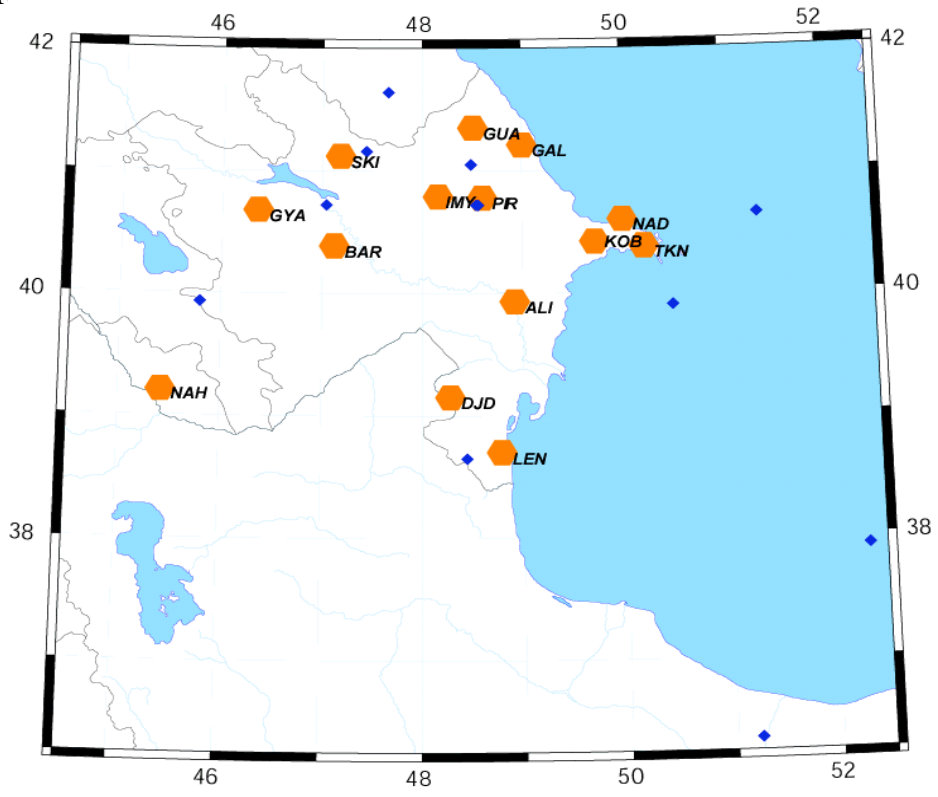

Figure 9. Station locations are orange and events blue. 
Table 6. Digital Station Locations

\begin{tabular}{|l|lll|}
\hline \multicolumn{4}{c|}{ Azerbaijan Analog Stations } \\
\hline \multicolumn{1}{|c|}{ Station } & \multicolumn{1}{c|}{ ID* } & \multicolumn{2}{c|}{ Latitude Longitude } \\
\hline ALI-BAYRAML & ALI & 39.95 & 49.00 \\
\hline BERDE & BRD & 40.26 & 47.18 \\
\hline GALA & GAL & 40.41 & 50.16 \\
\hline GANJA & GAN & 40.65 & 46.32 \\
\hline JELABAD & GLB & 39.24 & 48.39 \\
\hline GOBU & GOB & 40.40 & 49.73 \\
\hline ISMAILLI & IML & 40.79 & 48.18 \\
\hline LENKORAN & LRK & 38.71 & 48.77 \\
\hline NAKHICHEVAN & NAX & 39.17 & 45.49 \\
\hline NARDARAN & NDR & 40.58 & 49.99 \\
\hline PIRGULU & PQL & 40.79 & 48.59 \\
\hline GUBA & GUB & 41.36 & 48.49 \\
\hline SHEKI & SEK & 41.21 & 47.20 \\
\hline & SIZ & 41.08 & 48.9 \\
\hline
\end{tabular}

*Station ID's created, none exist.

\section{- Eastern Turkey Network (IRIS)}

Data from the Eastern Turkey network were obtained from the Kandilli Observatory and Earthquake Research Institute National Earthquake Monitoring Centre. These data are in SAC format. Overall, twenty-two stations recorded thirty-seven events between the years 1979-2004. Some of the data were excluded because there was missing header information. This dataset includes earthquakes magnitude 4.0 and greater. Figure 9 is a plot of the stations in the Eastern Turkey Network, and events archived. Only the eastern part of Turkey was included in the map. Table 6 lists station locations.

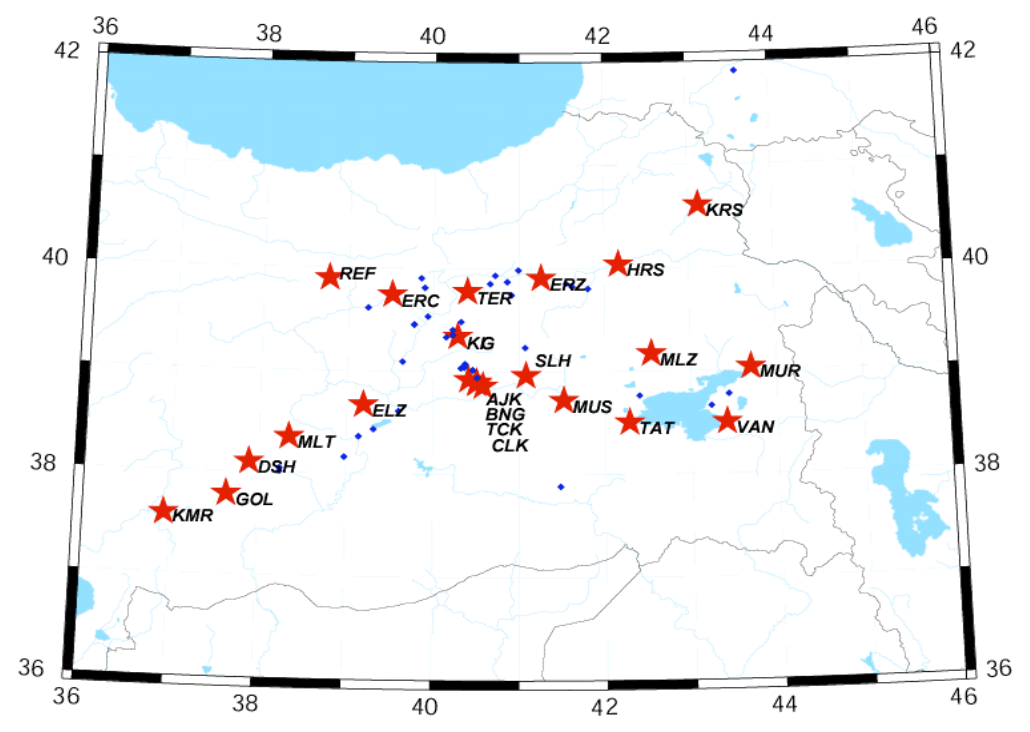

Figure 9. Stations in the Eastern Turkey Network (stars), and events (blue dots) 
Table 6. Station locations.

\begin{tabular}{|c|c|c|c|}
\hline \multicolumn{4}{|c|}{ Eastern Turkey Stations } \\
\hline Station & ID & Latitude & Longitude \\
\hline ATAPARK JANDARMA KRK.BATI BINGOL & AJK & $38.919 \mathrm{~N}$ & $40.410 \mathrm{E}$ \\
\hline BINGOL BINGOL BAY. VE ISKAN MUD.GU & BNG & $38.897 \mathrm{~N}$ & $40.503 \mathrm{E}$ \\
\hline BINGOL KARAYOLLARI 84 SUBE SEF. & TCK & $38.885 \mathrm{~N}$ & $40.504 \mathrm{E}$ \\
\hline CELTIKSUYU KOYU-TELEKOM BINASI & ÇLK & $38.858 \mathrm{~N}$ & $40.577 \mathrm{E}$ \\
\hline DOGANSEHIR METEOROLOJI MUDURLUGU & DSH & $38.096 \mathrm{~N}$ & 37.887E \\
\hline ELAZIG BAYINDIRLIK MUDURLUGU & ELZ & $38.672 \mathrm{~N}$ & 39.193E \\
\hline ERZINCAN BAYINDIRLIK MUDURLUGU & ERC & $39.743 \mathrm{~N}$ & $39.512 \mathrm{E}$ \\
\hline ERZURUM METEOROLOJI MUDURLUGU & ERZ & $39.906 \mathrm{~N}$ & 41.256E \\
\hline GOLBASI DEVLET HASTANESI & GOL & $37.781 \mathrm{~N}$ & $37.641 \mathrm{E}$ \\
\hline HORASAN METEOROLOJI MUDURLUGU & HRS & $40.040 \mathrm{~N}$ & $42.170 \mathrm{E}$ \\
\hline KARS BAYINDIRLIK VE ISKAN MUDURLUGU & KRS & $40.620 \mathrm{~N}$ & $48.07 \mathrm{E}$ \\
\hline KIGI METEOROLOJI MUDURLUGU & KIG & $39.340 \mathrm{~N}$ & $40.280 \mathrm{E}$ \\
\hline K.MARAS BAYINDIRLIK & KMR & $37.580 \mathrm{~N}$ & $36.931 \mathrm{E}$ \\
\hline MALATYA BAYINDIRLIK VE ISKAN MUDURLUGU & MLT & $38.350 \mathrm{~N}$ & $38.340 \mathrm{E}$ \\
\hline MALAZGIRT METEOROLOJI MUDURLUGU & MLZ & $39.170 \mathrm{~N}$ & $42.540 \mathrm{E}$ \\
\hline MURADIYE KAYMAKAMLIK BINASI & MUR & $39.030 \mathrm{~N}$ & $43.700 \mathrm{E}$ \\
\hline MUS BAYINDIRLIK MUDURLUGU & MUS & $38.720 \mathrm{~N}$ & $41.520 \mathrm{E}$ \\
\hline REFAHIYE KAYMAKAMLIK BINASI & REF & $39.901 \mathrm{~N}$ & $38.769 \mathrm{E}$ \\
\hline SOLHAN OGRETMEN EVI & SLH & $38.960 \mathrm{~N}$ & 41.080E \\
\hline TATVAN TATVAN METEOROLOJIISTASYONU & TAT & $38.503 \mathrm{~N}$ & $42.281 \mathrm{E}$ \\
\hline TERCAN METEOROLOJI MUDURLUGU & TER & $39.775 \mathrm{~N}$ & 40.391E \\
\hline VAN BAYINDIRLIK MUDURLUGU & VAN & $38.501 \mathrm{~N}$ & $43.407 \mathrm{E}$ \\
\hline
\end{tabular}

\section{- Caucasus Array (IRIS)}

Data were requested from IRIS using JWeed version 2.92, software distributed through IRIS, for the years 1990-2004. The search was limited to events that occurred between Latitude $40^{\circ}$ to $51^{\circ}$ and Longitude $38^{\circ}$ to $45^{\circ}$. During the time period, three different networks recorded in the area: the Global Seismograph Network (II \& IU), Caucasus Array (CS), and Eastern Turkey Seismic Experiment (XG). The Global Seismograph Network II had one station located in the region of interest, KIV, and IU had another station GNI, both utilized broadband instruments. The Caucasus Array recorded the years 1992-1994 using short period seismometers at five stations. The Eastern Turkey Seismic Experiment had 16 stations located in our study area and recorded the years 1999-2001 on broadband instruments. There were 1,845 earthquakes recorded on one or more stations from IRIS. Figure 10 shows station locations and Figure 11 shows event locations. Table 7 lists station locations. 


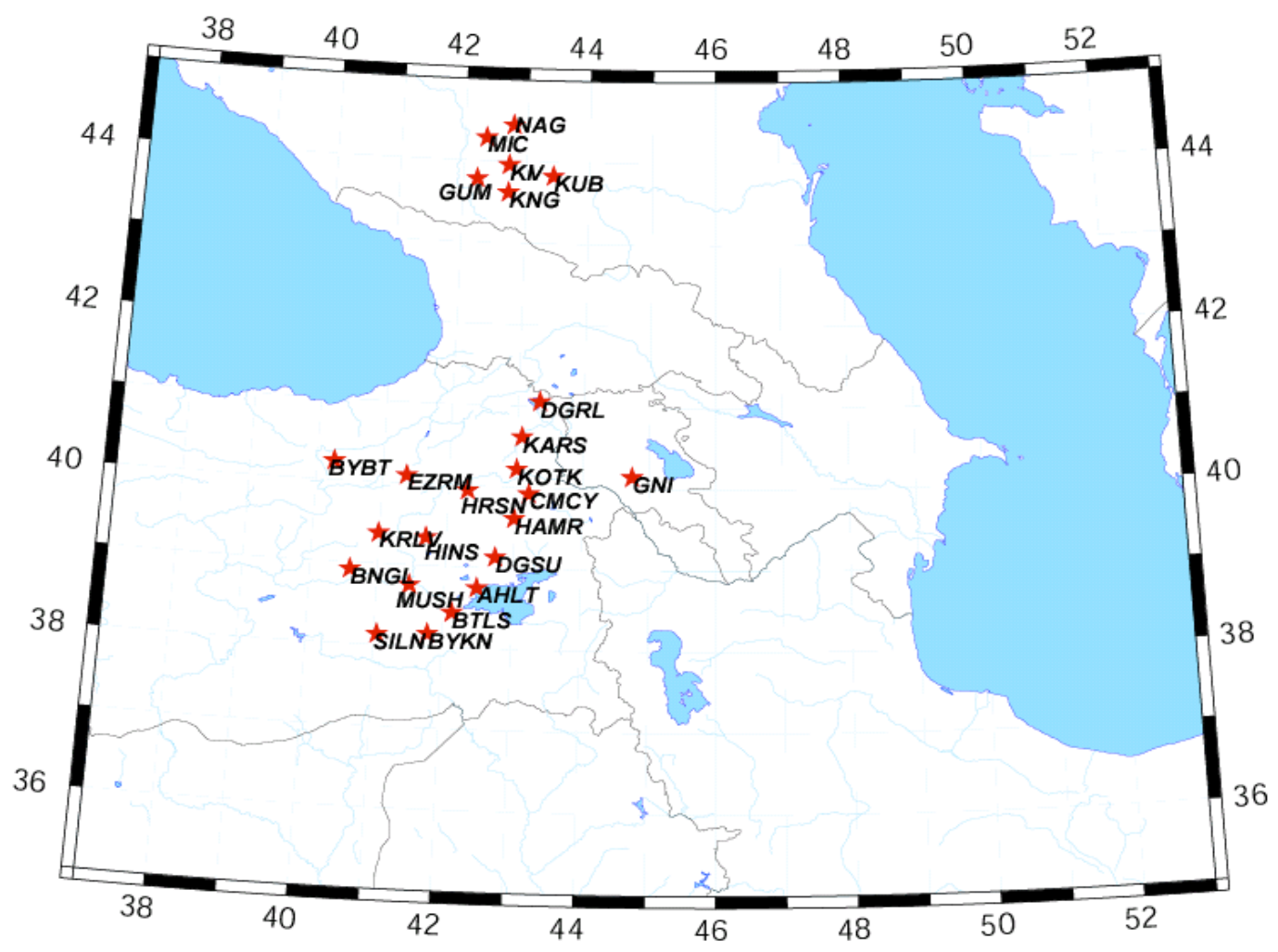

Figure 10. IRIS station locations.

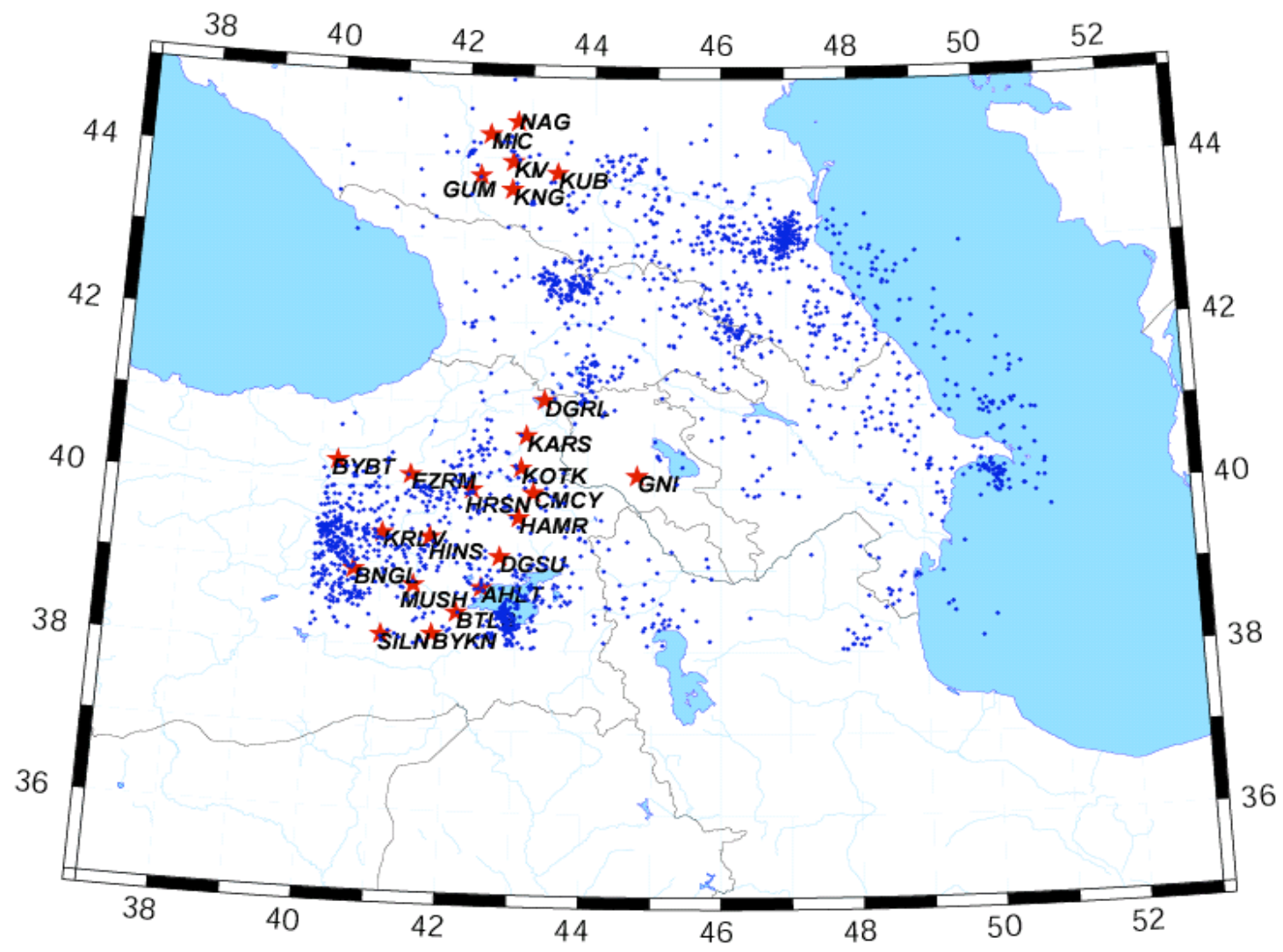

Figure 11. Event locations 
Table 7. Station locations

\begin{tabular}{|c|c|c|c|}
\hline Station & ID & Latitude & Longitude \\
\hline & BYBT & 40.23535 & 40.26550 \\
\hline & BNGL & 38.91949 & 40.59658 \\
\hline & KRLV & 39.37462 & 40.98811 \\
\hline & SILN & 38.13523 & 41.04099 \\
\hline & EZRM & 40.10351 & 41.36365 \\
\hline & MUSH & 38.75712 & 41.48314 \\
\hline & HINS & 39.34886 & 41.69720 \\
\hline & BYKN & 38.16654 & 41.78233 \\
\hline & BTLS & 38.43108 & 42.12383 \\
\hline & GUM & 43.7755 & 42.22800 \\
\hline & HRSN & 39.94531 & 42.28736 \\
\hline & MIC & 44.281 & 42.34800 \\
\hline & AHLT & 38.7481 & 42.47701 \\
\hline & KIV & 43.9557 & 42.72380 \\
\hline & KNG & 43.6178 & 42.72520 \\
\hline & DGSU & 39.13115 & 42.72975 \\
\hline & NAG & 44.4443 & 42.77500 \\
\hline & HAMR & 39.61356 & 42.99273 \\
\hline & KOTK & 40.22266 & 43.00941 \\
\hline & KARS & 40.6222 & 43.06865 \\
\hline & CMCY & 39.92054 & 43.19677 \\
\hline & DGRL & 41.05684 & 43.32675 \\
\hline & KUB & 43.8292 & 43.42900 \\
\hline & GNI & 40.148 & 44.74100 \\
\hline
\end{tabular}

- Historical Waveforms

Some historical waveforms have been digitized, and the digital data are in the process of being converted to SAC.

- Global Seismograph Network II \& IU (IRIS) 


\section{Appendix A: Removing Instrument Response}

This section gives a brief overview of what instrument response is and how to remove it from the waveform. It also describes in detail, the instrument removal process for each subset of the dataset, along with the software used.

Generally speaking, a raw waveform is the convolution of the signal from the seismic source, instrument response, and the way in which the waves propagate through the ground. For most seismometers the instrument response is known. This response can be determined simply by exposing the instrument to a delta function (boxcar in frequency domain) and seeing how it is recorded by the seismometer. Removing the instrument response can most easily be thought of as a filter. This "filter" is described by the response curves of both the amplitude and phase in the spectral domain or represented by poles and zeros. The poles and zeros describe the shape of the response curve with a series of numbers representing the positions of the poles and zeros in the complex plane.

\section{- Instrument removal from IRIS data}

The IRIS data were received in SEED (The Standard for the Exchange of Earthquake Data) format. The SEED file not only contain waveform data, but poles and zeros files, that can be utilized by SAC to deconvolve the instrument response. A program distributed and/or written by IRIS, rdseed, allows the user to remove data from SEED and put it in other useful formats, and in this case, SAC. Options $p$ and $d$ in rdseed were used to attain the poles and zeros files $(-p)$ as well as the waveforms (-d). After, the data were extracted from SEED format using rdseed; the $\mathrm{SAC}$ transfer function was used to remove the instrument response. In order, to preserve the data contained in the seismograms, filters were not applied to the broadband instruments. Some of the short period instruments needed to be filtered due to the long period noise in the seismogram and were bandpassed using a Butterworth filter with a corner frequencies at 0.2 and $20 \mathrm{HZ}$.

Adding the instrument response to a delta function and then removing it completed validation of the SAC transfer function. This is represented in the figure below, the red plot is the delta function, and the black represents the convolution then deconvolution of the instrument response. The result was what was expected in all cases, a delta function. The small amplitude sinusoids are a numerical artifact.

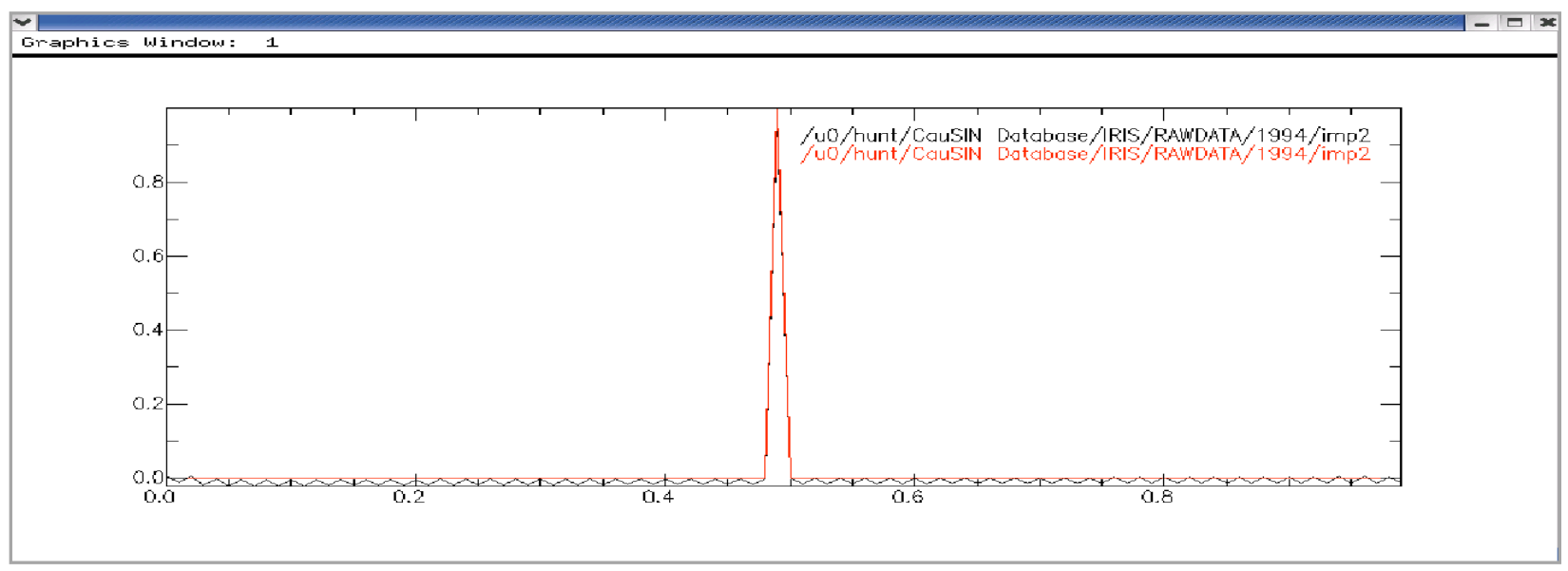




\section{- Instrument removal from Armenian Weak Motion}

The Armenian weak motion data were received in ASCII format. It was converted to SAC format using the readalpha command in SAC. A polezero file for the instruments in the Network was never obtained. However, a plot of the amplitude spectrum was given as a representation of the SM-3KV instrument response. In order to proceed, the phase shift was assumed to be zero. Deconvolution of the waveform was performed in the frequency domain, using only the waveform's amplitude and the amplitude representing the instrument's response. The units were then verified to be $\mathrm{m} / \mathrm{s}$ and then changed to $\mathrm{cm} / \mathrm{s}$.

\section{- Instrument response for the Azerbaijan Analog Network}

Instrument response was never obtained for the Azerbaijan Analog Network.

\section{- Instrument response for the Eastern Turkey Network}

The instrument response was already removed by Kandilli Observatory and Earthquake Research Institute National Earthquake Monitoring Centre before it was added to the database.

- Instrument response for the Azerbaijan Kinemetrics Network

The broadband network run by Azerbaijan is a new Kinemetrics Network. The instrument types were requested along with the longer waveform records.

\section{- Instrument response for the Georgian Weak Motion Network}

The instrument response for the Georgian Weak Motion Network was given in polezero format and the data were reported to be in $\mathrm{nm} / \mathrm{s}$ after the instrument response was removed. In the CORRECTED directory in the database the units are $\mathrm{cm} / \mathrm{s}$. There are some discrepancies for the instrument response for DELI and GORI; they are running Guralp 40Ts instruments and the polezero files seemed to give erroneous results. A similar type of instrument was found on Iris's website at www.passcal.nmt.edu/instrumentation/Sensor/Response/response table.html, however the poles and zeros were transposed but the numbers were the same. It was assumed that an error was made and the IRIS instrument response was used. The instrument response was removed in SAC using the "transfer" function. 


\section{Appendix B: Quality Assurance of Data}

\section{- Signal to Noise Ratio}

A program is being written to process data in such a way that detects when the signal to noise ratio is within acceptable parameters and stores this information in the SAC header. This program will separate out poor quality data with a low signal to noise ratio. A sample representing high signal to noise ratio is plotted below in SAC. The frequency range that is of interest is from $0.2 \mathrm{~Hz}$ to $6 \mathrm{~Hz}$. This record is from station KIV recording on channel BHE.

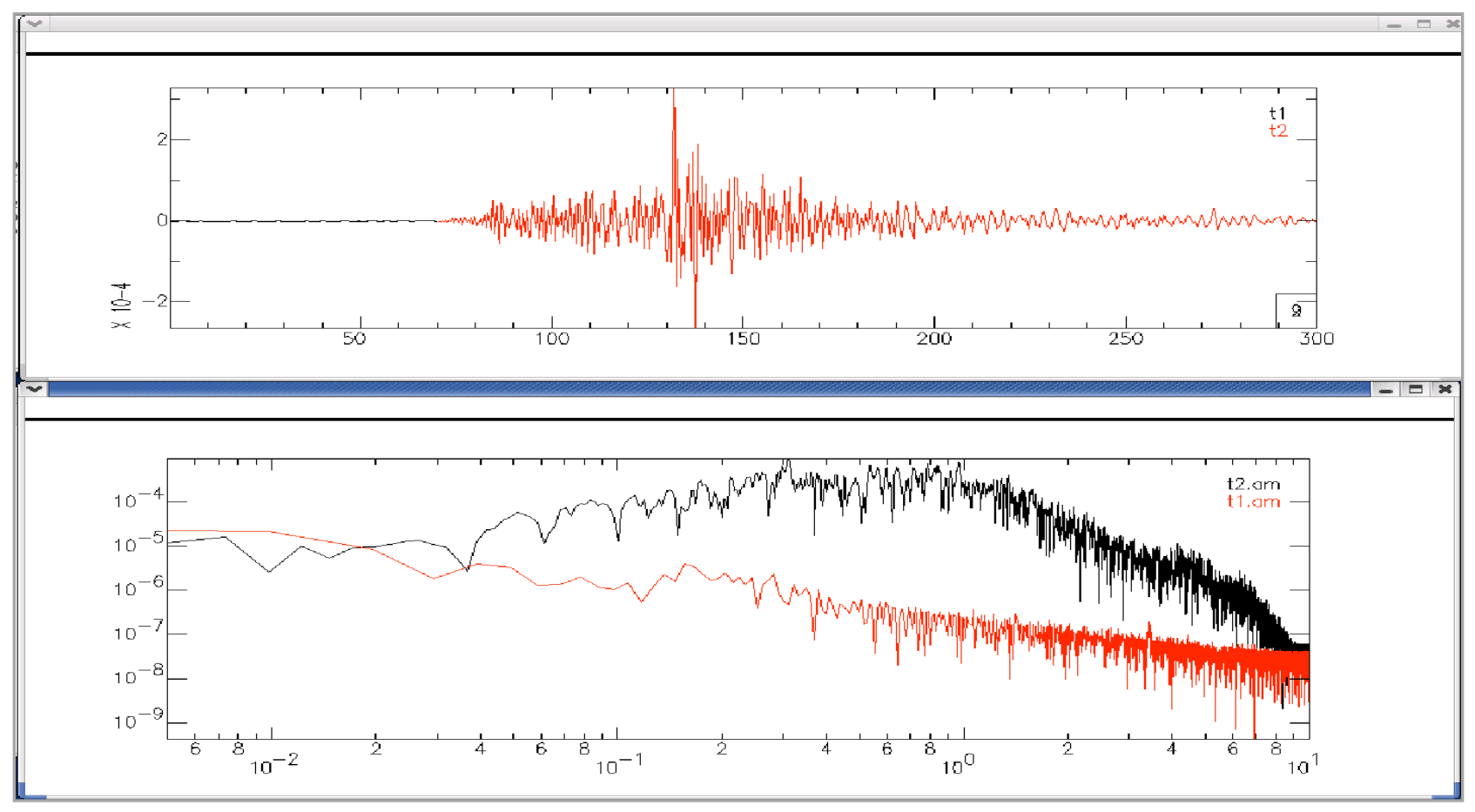




\section{Appendix C: Events Archived by the CauSIN project}

\begin{tabular}{|c|c|c|c|c|}
\hline YYMMDDHHMMSS & Latitude & Longitude & Mag. & Stations with records \\
\hline 000103135457 & 42.07 & 40.39 & 3.6 & AHLT BNGL BYBT BYKN CMCY DGSU EZRM GNI HAMR HINS HRSN KARS KIV KOTK MUSH SILN \\
\hline 000108183319 & 45.19 & 42.73 & 4.3 & AHLT BNGL BYBT BYKN CMCY DGSU EZRM GNI HAMR HINS HRSN KARS KIV KOTK KRLV MUSH SILN \\
\hline 000115220810 & 40.33 & 39.28 & 3.6 & AHLT BNGL BYBT BYKN CMCY DGSU EZRM GNI HAMR HINS HRSN KARS KIV KOTK KRLV MUSH SILN \\
\hline 000116120338 & 40.79 & 39.04 & 2.9 & AHLT BNGL BYBT BYKN CMCY DGSU EZRM GNI HAMR HINS HRSN KARS KIV KOTK KRLV MUSH SILN \\
\hline 000119141535 & 41.3 & 38.72 & 4 & AHLT BNGL BYBT BYKN CMCY DGSU EZRM GNI HAMR HINS HRSN KARS KIV KOTK KRLV MUSH SILN \\
\hline 000125181317 & 42.26 & 39.91 & 3.6 & AHLT BNGL BYBT BYKN CMCY DGSU EZRM GNI HAMR HINS HRSN KARS KIV KOTK KRLV MUSH SILN \\
\hline 000126132857 & 40.46 & 39.13 & 3.2 & AHLT BNGL BYBT BYKN CMCY DGSU EZRM GNI HAMR HINS HRSN KARS KIV KOTK KRLV MUSH SILN \\
\hline 000127101840 & 41.99 & 40.2 & 3.8 & AHLT BNGL BYBT BYKN CMCY DGSU EZRM GNI HAMR HINS HRSN KARS KIV KOTK MUSH SILN \\
\hline 000128001528 & 40.2 & 39.23 & 3.5 & AHLT BNGL BYBT BYKN CMCY DGSU EZRM GNI HAMR HINS HRSN KARS KIV KOTK MUSH SILN \\
\hline 000128232709 & 46.93 & 43.14 & 4.3 & AHLT BNGL BYBT BYKN CMCY EZRM GNI HAMR HINS HRSN KARS KIV KOTK KRLV MUSH SILN \\
\hline 000201194622 & 41.12 & 39.07 & 4.4 & AHLT BNGL BYBT BYKN CMCY EZRM GNI HAMR HINS HRSN KARS KIV KOTK MUSH SILN \\
\hline 000201201212 & 41.13 & 39.46 & 3.1 & AHLT BNGL BYBT BYKN CMCY EZRM GNI HAMR HINS HRSN KARS KIV KOTK MUSH SILN \\
\hline 000206164415 & 46.86 & 42.82 & 4 & AHLT BNGL BYBT BYKN CMCY EZRM GNI HAMR HINS HRSN KARS KIV KOTK MUSH SILN \\
\hline 000210093732 & 46.9 & 41.8 & 4.1 & AHLT BNGL BYBT BYKN CMCY EZRM GNI HAMR HINS HRSN KARS KIV KOTK MUSH SILN \\
\hline 000210145338 & 40.74 & 39.06 & 3.1 & AHLT BNGL BYBT BYKN CMCY EZRM GNI HAMR HINS HRSN KARS KIV KOTK MUSH SILN \\
\hline 000213013253 & 40.34 & 39.38 & 3.3 & AHLT BNGL BYBT BYKN CMCY EZRM GNI HAMR HINS HRSN KARS KIV KOTK MUSH SILN \\
\hline 000216061244 & 42.47 & 41.13 & 3.6 & AMB \\
\hline 000216083857 & 42.37 & 40.93 & 3.6 & AMB \\
\hline 000223082138 & 46.03 & 42.52 & 4 & AHLT BNGL BYBT BYKN CMCY EZRM GNI HAMR HINS HRSN KARS KIV KOTK MUSH SILN \\
\hline 000225160820 & 40.24 & 39.18 & 3.1 & AHLT BNGL BYBT BYKN CMCY EZRM GNI HAMR HINS HRSN KARS KIV KOTK MUSH SILN \\
\hline 000229013421 & 47.27 & 42.93 & 4 & AHLT BNGL BYBT BYKN CMCY EZRM GNI HAMR HINS HRSN KARS KIV KOTK MUSH SILN \\
\hline 000302103220 & 44.9 & 43.41 & 4.1 & AHLT BNGL BYBT BYKN CMCY EZRM GNI HAMR HINS HRSN KARS KIV KOTK MUSH SILN \\
\hline 000307142253 & 46.36 & 43.25 & 4.4 & AHLT BNGL BYBT BYKN CMCY DGSU EZRM GNI HAMR HINS HRSN KARS KIV KOTK KRLV MUSH SILN \\
\hline 000308164422 & 45.62 & 43.32 & 4.1 & AHLT BNGL BYBT BYKN CMCY DGSU EZRM GNI HAMR HINS HRSN KARS KIV KOTK KRLV MUSH SILN \\
\hline 000309142942 & 47.65 & 41.01 & 4 & AHLT BNGL BYBT BYKN CMCY EZRM GNI HINS HRSN KARS KIV KOTK KRLV MUSH SILN \\
\hline 000309142943 & 47.15 & 41.25 & 3.6 & PAR \\
\hline 000310012234 & 47.71 & 42.44 & 4.2 & AHLT BNGL BYBT BYKN CMCY EZRM GNI HINS HRSN KARS KIV KOTK KRLV MUSH SILN \\
\hline 000312124803 & 40 & 38.79 & 3.2 & AHLT BNGL BYKN CMCY DGRL EZRM GNI HINS HRSN KARS KIV KOTK KRLV MUSH SILN \\
\hline 000312134504 & 40.26 & 38.82 & 3.2 & AHLT BNGL BYKN CMCY DGRL EZRM GNI HINS HRSN KARS KIV KOTK KRLV MUSH SILN \\
\hline 000313170316 & 43.77 & 38.78 & 3.8 & AHLT BNGL BYKN CMCY DGRL EZRM GNI HINS HRSN KARS KIV KOTK KRLV MUSH SILN \\
\hline 000317075409 & 42.45 & 40.67 & 3.5 & AMB \\
\hline 000317121406 & 42.62 & 39.75 & 3.8 & AMB \\
\hline 000317121415 & 42.52 & 40.37 & 4 & AHLT BNGL BYKN CMCY DGRL EZRM GNI HINS HRSN KARS KIV KOTK KRLV MUSH SILN \\
\hline 000318040951 & 42.38 & 40.48 & 4.6 & AHLT BNGL BYKN CMCY DGRL EZRM GNI HINS HRSN KARS KIV KOTK KRLV MUSH SILN \\
\hline 000319072034 & 41.27 & 43.82 & 4 & AHLT BNGL BYKN CMCY DGRL EZRM GNI HINS HRSN KARS KIV KOTK KRLV MUSH SILN \\
\hline 000320064522 & 42.25 & 40.48 & 4.2 & AMB \\
\hline 000320064532 & 42.4 & 40.44 & 4.1 & AHLT BNGL BYKN CMCY DGRL EZRM GNI HINS HRSN KARS KIV KOTK KRLV MUSH SILN \\
\hline 000321064652 & 50.91 & 39.74 & 3.8 & AHLT BNGL BYKN CMCY DGRL EZRM GNI HINS HRSN KARS KIV KOTK KRLV MUSH SILN \\
\hline 000321140740 & 48.2 & 40.05 & 5.2 & AHLT BNGL BYKN CMCY DGRL EZRM GNI HINS HRSN KARS KIV KOTK KRLV MUSH SILN \\
\hline 000323060852 & 40.4 & 38.76 & 3.2 & AHLT BNGL BYKN CMCY DGRL EZRM GNI HINS HRSN KARS KIV KOTK KRLV MUSH SILN \\
\hline 000326133045 & 46.36 & 42.47 & 4.1 & AHLT BNGL BTLS BYKN CMCY DGRL EZRM GNI HINS HRSN KARS KIV KOTK KRLV MUSH SILN \\
\hline 000328182727 & 41.47 & 38.79 & 3.9 & AHLT BNGL BTLS BYKN CMCY DGRL EZRM GNI HINS HRSN KARS KIV KOTK KRLV MUSH SILN \\
\hline 000330152702 & 41.62 & 38.98 & 3.5 & AHLT BNGL BTLS BYKN CMCY DGRL EZRM GNI HINS HRSN KARS KIV KOTK KRLV MUSH SILN \\
\hline 000331122450 & 43 & 39.49 & 4 & AHLT BNGL BTLS BYKN CMCY DGRL EZRM GNI HINS HRSN KARS KIV KOTK KRLV MUSH SILN \\
\hline 000407024649 & 46.93 & 42.94 & 4.2 & AHLT BNGL BTLS BYKN CMCY DGRL EZRM GNI HINS HRSN KARS KIV KRLV MUSH SILN \\
\hline
\end{tabular}




\begin{tabular}{|c|c|c|c|c|}
\hline 000410013916 & 40.22 & 38.53 & 3.5 & AHLT BNGL BTLS BYKN CMCY DGRL EZRM GNI HINS HRSN KARS KIV KRLV MUSH SILN \\
\hline 000410034240 & 48.11 & 38.07 & 4.2 & AHLT BNGL BTLS BYKN CMCY DGRL EZRM GNI HINS HRSN KARS KIV KRLV MUSH SILN \\
\hline 000410123429 & 44.77 & 42.84 & 4 & AHLT BNGL BTLS BYKN CMCY DGRL EZRM GNI HINS HRSN KARS KIV KRLV MUSH SILN \\
\hline 000410211321 & 50.11 & 41.01 & 3.2 & AHLT BNGL BTLS BYKN CMCY DGRL EZRM GNI HINS HRSN KARS KIV KRLV MUSH SILN \\
\hline 000410213655 & 50.37 & 41.39 & 3.6 & AHLT BNGL BTLS BYKN CMCY DGRL EZRM GNI HINS HRSN KARS KIV KRLV MUSH SILN \\
\hline 000411235151 & 46.98 & 42.92 & 4 & AHLT BNGL BTLS BYKN CMCY DGRL EZRM GNI HINS HRSN KARS KIV KRLV MUSH SILN \\
\hline 000412000130 & 47.12 & 43.28 & 4.7 & AHLT BNGL BTLS BYKN CMCY DGRL EZRM GNI HINS HRSN KARS KIV KRLV MUSH SILN \\
\hline 000412224949 & 42.52 & 40.1 & 3.4 & AHLT BNGL BTLS BYKN CMCY DGRL EZRM GNI HINS HRSN KARS KIV KRLV MUSH PAR SILN \\
\hline 000412233827 & 46.92 & 39.94 & 4.4 & AHLT BNGL BTLS BYKN CMCY DGRL EZRM GNI HINS HRSN KARS KIV KRLV MUSH SILN \\
\hline 000413111248 & 45.18 & 43.52 & 3.7 & AHLT BNGL BTLS BYKN CMCY DGRL EZRM GNI HINS HRSN KARS KIV KRLV MUSH SILN \\
\hline 000415071521 & 47.03 & 43.09 & 4.7 & AHLT BNGL BTLS BYKN CMCY DGRL EZRM GNI HINS HRSN KARS KIV KRLV MUSH SILN \\
\hline 000415123932 & 50.25 & 40.71 & 4 & AHLT BNGL BTLS BYKN CMCY DGRL EZRM GNI HINS HRSN KARS KIV KRLV MUSH SILN \\
\hline 000415203918 & 45.89 & 42.18 & 4 & AHLT BNGL BTLS BYKN CMCY DGRL EZRM GNI HINS HRSN KARS KIV KRLV MUSH SILN \\
\hline 000422084635 & 48.71 & 42.04 & 4.9 & AHLT BNGL BTLS BYKN CMCY DGRL EZRM GNI HINS HRSN KIV KRLV MUSH SILN \\
\hline 000422084640 & 48.23 & 41.27 & 3.8 & AMB \\
\hline 000422220424 & 50.2 & 41.8 & 3.6 & AHLT BNGL BTLS BYKN CMCY DGRL EZRM GNI HINS HRSN KIV KRLV MUSH SILN \\
\hline 000423160627 & 46.76 & 43.43 & 4 & AHLT BNGL BTLS BYKN CMCY DGRL EZRM GNI HINS HRSN KIV KRLV MUSH SILN \\
\hline 000424041732 & 46.61 & 43.25 & 3.8 & AHLT BNGL BTLS BYKN CMCY DGRL EZRM GNI HINS HRSN KIV KRLV MUSH SILN \\
\hline 000424044009 & 50.06 & 41 & 3.7 & AHLT BNGL BTLS BYKN CMCY DGRL EZRM GNI HINS HRSN KIV KRLV MUSH SILN \\
\hline 000424070935 & 50.01 & 41.07 & 3.7 & AHLT BNGL BTLS BYKN CMCY DGRL EZRM GNI HINS HRSN KIV KRLV MUSH SILN \\
\hline 000424154516 & 50.1 & 40.98 & 3.8 & AHLT BNGL BTLS BYKN CMCY DGRL EZRM GNI HINS HRSN KIV KRLV MUSH SILN \\
\hline 000424170457 & 50.37 & 41.05 & 3.6 & AHLT BNGL BTLS BYKN CMCY DGRL EZRM GNI HINS HRSN KIV KRLV MUSH SILN \\
\hline 000425012758 & 50.26 & 41 & 3.8 & AHLT BNGL BTLS BYKN CMCY DGRL EZRM GNI HINS HRSN KIV KRLV MUSH SILN \\
\hline 000425061254 & 46.26 & 41.86 & 4.1 & AHLT BNGL BTLS BYKN CMCY DGRL EZRM GNI HINS HRSN KIV KRLV MUSH SILN \\
\hline 000425084951 & 50.05 & 40.88 & 3.8 & AHLT BNGL BTLS BYKN CMCY DGRL EZRM GNI HINS HRSN KIV KRLV MUSH SILN \\
\hline 000428221449 & 40.85 & 38.62 & 3.4 & AHLT BNGL BTLS BYKN CMCY DGRL EZRM GNI HINS HRSN KIV KRLV MUSH SILN \\
\hline 000502161939 & 45.89 & 41.23 & 4.4 & AHLT BNGL BTLS BYKN CMCY DGRL EZRM GNI HINS HRSN KIV KRLV MUSH SILN \\
\hline 000506220157 & 48.07 & 41.57 & 4.5 & AHLT BNGL BTLS BYKN CMCY DGRL EZRM GNI HINS HRSN KIV KRLV MUSH SILN \\
\hline 000507023926 & 50.75 & 40.93 & 3.6 & AHLT BNGL BTLS BYKN CMCY DGRL EZRM GNI HINS HRSN KIV KRLV MUSH SILN \\
\hline 000507045343 & 45.71 & 42.49 & 4.1 & AHLT BNGL BTLS BYKN CMCY DGRL EZRM GNI HINS HRSN KIV KRLV MUSH SILN \\
\hline 000519053119 & 48.42 & 38.54 & 3.9 & AHLT BNGL BTLS BYKN CMCY DGRL EZRM GNI HINS HRSN KIV KRLV MUSH SILN \\
\hline 000519123101 & 44.3 & 42.5 & 4.3 & AHLT BNGL BTLS BYKN CMCY DGRL EZRM GNI HINS HRSN KIV KRLV SILN \\
\hline 000530020057 & 44.95 & 42.56 & 4.3 & AHLT BNGL BTLS BYKN CMCY DGRL EZRM GNI HINS HRSN KRLV SILN \\
\hline 000602195922 & 46.96 & 43.12 & 4.2 & AHLT BNGL BTLS BYKN CMCY DGRL EZRM GNI HINS HRSN KIV KRLV SILN \\
\hline 000603054540 & 45.9 & 43.7 & 4 & AHLT BNGL BTLS BYKN CMCY DGRL EZRM GNI HINS HRSN KIV KRLV SILN \\
\hline 000605235928 & 42.5 & 38.71 & 3.8 & AHLT BNGL BTLS BYKN CMCY DGRL EZRM GNI HINS HRSN KIV KRLV SILN \\
\hline 000606012607 & 42.44 & 38.73 & 3.1 & AHLT BNGL BTLS BYKN CMCY DGRL EZRM GNI HINS HRSN KIV KRLV SILN \\
\hline 000609161059 & 49.63 & 41.87 & 3.8 & AHLT BNGL BTLS BYKN CMCY DGRL EZRM GNI HINS HRSN KIV KRLV SILN \\
\hline 000610075355 & 48.22 & 39.14 & 4.4 & AMB \\
\hline 000615203845 & 41.57 & 39.08 & 3.8 & AHLT BNGL BTLS BYKN CMCY DGRL EZRM GNI HINS HRSN KIV KRLV SILN \\
\hline 000617110838 & 44.21 & 43.86 & 4.2 & AHLT BNGL BTLS BYKN CMCY DGRL EZRM GNI HINS HRSN KIV KRLV SILN \\
\hline 000617173518 & 44.18 & 43.49 & 4.2 & AHLT BNGL BTLS BYKN CMCY DGRL EZRM GNI HINS HRSN KIV KRLV SILN \\
\hline 000627220413 & 40.14 & 38.88 & 3.7 & BNGL BTLS BYBT BYKN CMCY DGRL EZRM GNI HRSN KIV KRLV SILN \\
\hline 000629114337 & 46.32 & 42.99 & 4.2 & BNGL BTLS BYBT BYKN CMCY DGRL EZRM GNI HRSN KIV KRLV SILN \\
\hline 000629221527 & 46.51 & 43.68 & 4.4 & BNGL BTLS BYBT BYKN CMCY DGRL EZRM GNI HRSN KIV KRLV SILN \\
\hline 000630170526 & 45.81 & 43.26 & 3.8 & BNGL BTLS BYBT BYKN CMCY DGRL EZRM GNI HRSN KIV KRLV SILN \\
\hline 000702114244 & 50.02 & 40.94 & 4.1 & BNGL BTLS BYBT BYKN CMCY EZRM GNI HRSN KIV KRLV SILN \\
\hline 000703070650 & 49.83 & 42.08 & 4.1 & BNGL BTLS BYBT BYKN CMCY EZRM GNI HRSN KIV KRLV SILN \\
\hline 000703153554 & 41.62 & 39.17 & 3.4 & BNGL BTLS BYBT BYKN CMCY EZRM GNI HRSN KIV KRLV SILN \\
\hline 000705172923 & 47.57 & 41.99 & 4.6 & BNGL BTLS BYBT BYKN CMCY EZRM GNI HRSN KIV KRLV SILN \\
\hline 000708172127 & 42.97 & 38.25 & 3.6 & BNGL BTLS BYBT BYKN DGRL EZRM GNI HRSN KIV KRLV SILN \\
\hline 000709093604 & 40.76 & 39.26 & 3.6 & BNGL BTLS BYBT BYKN DGRL EZRM GNI HRSN KIV KRLV SILN \\
\hline
\end{tabular}




\begin{tabular}{|c|c|c|c|c|}
\hline 000709131255 & 47.32 & 43.01 & 4.4 & BNGL BTLS BYBT BYKN DGRL EZRM GNI HRSN KIV KRLV MUSH SILN \\
\hline 000710052957 & 42.29 & 39.02 & 3.2 & BNGL BTLS BYBT BYKN DGRL EZRM GNI HRSN KIV KRLV MUSH SILN \\
\hline 000714125852 & 47.17 & 41.97 & 4.6 & BNGL BTLS BYBT DGRL EZRM GNI HRSN KIV KRLV MUSH SILN \\
\hline 000717045454 & 41.54 & 39.31 & 3.2 & BNGL BTLS BYBT DGRL EZRM GNI HRSN KIV KRLV MUSH \\
\hline 000723102050 & 40.51 & 39.33 & 3.2 & BNGL BTLS BYBT DGRL EZRM GNI HRSN KIV KRLV MUSH \\
\hline 000726082650 & 46.98 & 43.05 & 4 & BNGL BTLS BYBT DGRL EZRM GNI HRSN KIV KRLV MUSH \\
\hline 000729064001 & 42.41 & 39.1 & 3.5 & BNGL BTLS BYBT DGRL EZRM GNI HRSN KIV KRLV MUSH \\
\hline 000730061544 & 40.33 & 39.21 & 2.9 & BNGL BTLS BYBT DGRL EZRM GNI HRSN KIV KRLV MUSH \\
\hline 000730201859 & 42.6 & 40.44 & 3.5 & BNGL BTLS BYBT DGRL EZRM GNI HRSN KIV KRLV MUSH \\
\hline 000731233428 & 44.62 & 38.22 & 3.9 & AMB \\
\hline 000807014330 & 41.96 & 40.22 & 3.8 & BNGL BTLS BYBT DGRL EZRM GNI HRSN KIV KRLV MUSH \\
\hline 000807040129 & 47.12 & 43.14 & 4.3 & BNGL BTLS BYBT DGRL EZRM GNI HRSN KIV KRLV MUSH \\
\hline 000819084535 & 44.17 & 41.1 & 3.7 & AMB \\
\hline 000819212617 & 41.44 & 39.81 & 4.6 & BNGL BTLS BYBT DGRL EZRM GNI HRSN KIV KRLV MUSH \\
\hline 000825170201 & 46.97 & 43.08 & 4.1 & BNGL BTLS BYBT DGRL EZRM GNI HRSN KIV KRLV MUSH \\
\hline 000825192711 & 42.61 & 39.75 & 3.3 & BNGL BTLS BYBT DGRL EZRM GNI HRSN KIV KRLV MUSH \\
\hline 000830181936 & 45.53 & 42.98 & 4.4 & BNGL BTLS BYBT DGRL EZRM GNI HRSN KIV KRLV MUSH \\
\hline 000901213318 & 43.39 & 39.71 & 2.9 & BNGL BTLS BYBT DGRL EZRM GNI HRSN KIV KRLV MUSH \\
\hline 000904082919 & 41.65 & 38.95 & 3.5 & BNGL BTLS BYBT DGRL EZRM GNI HRSN KIV KRLV MUSH \\
\hline 000904163034 & 42.12 & 40.3 & 3 & BNGL BTLS BYBT DGRL EZRM GNI HRSN KIV KRLV MUSH \\
\hline 000907073037 & 45.16 & 39.01 & 3.5 & AMB \\
\hline 000907073233 & 45.08 & 38.47 & 3.8 & BNGL BTLS BYBT DGRL EZRM GNI HRSN KIV KRLV MUSH \\
\hline 000909195238 & 46.6 & 42.03 & 4.6 & BNGL BTLS BYBT DGRL EZRM GNI HRSN KIV KRLV MUSH \\
\hline 000911234304 & 40.78 & 39.34 & 2.9 & BNGL BTLS BYBT DGRL EZRM GNI HRSN KIV KRLV MUSH \\
\hline 000912193437 & 42.68 & 44.26 & 4 & BNGL BTLS BYBT DGRL EZRM GNI HRSN KIV KRLV MUSH \\
\hline 000919094816 & 47.01 & 43.01 & 4.4 & BNGL BTLS BYBT DGRL EZRM GNI HRSN KIV KRLV MUSH \\
\hline 000922022730 & 47.12 & 43.38 & 4.3 & BNGL BTLS BYBT DGRL EZRM GNI HRSN KIV KRLV MUSH \\
\hline 000923204843 & 40.54 & 38.62 & 2.5 & BNGL BTLS BYBT DGRL EZRM GNI HRSN KIV KRLV MUSH \\
\hline 000924063440 & 47.37 & 42.47 & 4.7 & BNGL BTLS BYBT DGRL EZRM GNI HRSN KIV KRLV MUSH \\
\hline 000924063951 & 47.43 & 42.42 & 4.2 & BNGL BTLS BYBT DGRL EZRM GNI HRSN KIV KRLV MUSH \\
\hline 000924071211 & 43.48 & 39.28 & 3.9 & AMB \\
\hline 000924071214 & 43.72 & 39.49 & 3.9 & BNGL BTLS BYBT DGRL EZRM GNI HRSN KIV KRLV MUSH \\
\hline 000924073533 & 47.49 & 42.42 & 4.2 & BNGL BTLS BYBT DGRL EZRM GNI HRSN KIV KRLV MUSH \\
\hline 000927034358 & 49.59 & 41.09 & 4.7 & BNGL BTLS BYBT DGRL EZRM GNI HRSN KIV KRLV MUSH \\
\hline 001001010407 & 44.58 & 42.58 & 4 & BNGL BTLS BYBT DGRL EZRM GNI HRSN KIV KRLV MUSH \\
\hline 001016044845 & 43.12 & 38.64 & 3.4 & BNGL BTLS BYBT DGRL EZRM GNI HRSN KIV KRLV MUSH \\
\hline 001017234743 & 45.87 & 41.85 & 4 & BNGL BTLS BYBT DGRL EZRM GNI HRSN KIV KRLV MUSH \\
\hline 001021181221 & 43.93 & 42.83 & 4 & BNGL BTLS BYBT DGRL EZRM GNI HRSN KIV KRLV MUSH \\
\hline 001022131834 & 45 & 38.55 & 3.7 & PAR \\
\hline 001022131839 & 44.56 & 38.54 & 3.9 & BNGL BYBT DGRL EZRM GNI HRSN KIV KRLV MUSH \\
\hline 001027091906 & 42 & 44 & 4 & BNGL BYBT DGRL EZRM GNI HRSN KIV KRLV MUSH \\
\hline 001027210019 & 40.38 & 39.43 & 3.2 & BNGL BYBT DGRL EZRM GNI HRSN KIV KRLV MUSH \\
\hline 001028125615 & 50.52 & 40.22 & 3.9 & BNGL BYBT DGRL EZRM GNI HRSN KIV KRLV MUSH \\
\hline 001028174638 & 40.28 & 38.5 & 3 & BNGL BYBT DGRL EZRM GNI HRSN KIV KRLV MUSH \\
\hline 001028200019 & 46.5 & 39.9 & 4 & BNGL BYBT DGRL EZRM GNI HRSN KIV KRLV MUSH \\
\hline 001101095706 & 43.1 & 38.92 & 3.4 & BNGL BYBT DGRL EZRM GNI HINS HRSN KARS KIV KRLV \\
\hline 001101154418 & 43.19 & 39 & 3.6 & BNGL BYBT DGRL EZRM GNI HINS HRSN KARS KIV KRLV \\
\hline 001102095708 & 43.37 & 39.05 & 3.6 & BNGL BYBT DGRL EZRM GNI HINS HRSN KARS KIV KOTK KRLV \\
\hline 001102154416 & 43.93 & 39.16 & 3.6 & BNGL BYBT DGRL EZRM GNI HINS HRSN KARS KIV KOTK KRLV \\
\hline 001107094451 & 49.87 & 39.87 & 4.2 & AHLT BNGL BTLS BYBT CMCY DGRL DGSU EZRM GNI HAMR HINS HRSN KIV KOTK KRLV MUSH \\
\hline 001114055016 & 43.38 & 43.72 & 4 & AHLT BNGL BTLS BYBT CMCY DGRL DGSU EZRM GNI HAMR HINS HRSN KIV KOTK KRLV MUSH \\
\hline 001115150536 & 42.95 & 38.41 & 5.5 & AHLT BNGL BTLS BYBT CMCY DGRL DGSU EZRM GNI HAMR HINS HRSN KIV KOTK KRLV MUSH \\
\hline
\end{tabular}




\begin{tabular}{|c|c|c|c|c|}
\hline 001115151357 & 42.9 & 38.44 & 2.9 & AHLT BNGL BTLS BYBT CMCY DGRL DGSU EZRM GNI HAMR HINS HRSN KIV KOTK KRLV MUSH \\
\hline 001115151544 & 42.91 & 38.21 & 2.8 & AHLT BNGL BTLS BYBT CMCY DGRL DGSU EZRM GNI HAMR HINS HRSN KIV KOTK KRLV MUSH \\
\hline 001115151724 & 42.97 & 38.15 & 5 & AHLT BNGL BTLS BYBT CMCY DGRL DGSU EZRM GNI HAMR HINS HRSN KIV KOTK KRLV MUSH \\
\hline 001115152224 & 42.87 & 38.05 & 3 & AHLT BNGL BTLS BYBT CMCY DGRL DGSU EZRM GNI HAMR HINS HRSN KIV KOTK KRLV MUSH \\
\hline 001115153148 & 42.78 & 38.25 & 2.8 & AHLT BNGL BTLS BYBT CMCY DGRL DGSU EZRM GNI HAMR HINS HRSN KIV KOTK KRLV MUSH \\
\hline 001115154037 & 43.06 & 38.86 & 3 & AHLT BNGL BTLS BYBT CMCY DGRL DGSU EZRM GNI HAMR HINS HRSN KIV KOTK KRLV MUSH \\
\hline 001115160608 & 42.75 & 38.43 & 4.2 & PAR \\
\hline 001115160613 & 42.91 & 38.59 & 4.4 & AHLT BNGL BTLS BYBT CMCY DGRL DGSU EZRM GNI HAMR HINS HRSN KIV KOTK KRLV MUSH \\
\hline 001115160853 & 42.81 & 38.33 & 3.2 & AHLT BNGL BTLS BYBT CMCY DGRL DGSU EZRM GNI HAMR HINS HRSN KIV KOTK KRLV MUSH \\
\hline 001115161102 & 42.86 & 38.32 & 3 & AHLT BNGL BTLS BYBT CMCY DGRL DGSU EZRM GNI HAMR HINS HRSN KIV KOTK KRLV MUSH \\
\hline 001115163023 & 42.88 & 38.12 & 3.1 & AHLT BNGL BTLS BYBT CMCY DGRL DGSU EZRM GNI HAMR HINS HRSN KIV KOTK KRLV MUSH \\
\hline 001115164338 & 43.23 & 38.02 & 3.8 & AHLT BNGL BTLS BYBT CMCY DGRL DGSU EZRM GNI HAMR HINS HRSN KIV KOTK KRLV MUSH \\
\hline 001115164647 & 42.82 & 38.26 & 2.8 & AHLT BNGL BTLS BYBT CMCY DGRL DGSU EZRM GNI HAMR HINS HRSN KIV KOTK KRLV MUSH \\
\hline 001115170722 & 43.01 & 38.25 & 3.5 & AHLT BNGL BTLS BYBT CMCY DGRL DGSU EZRM GNI HAMR HINS HRSN KIV KOTK KRLV MUSH \\
\hline 001115171022 & 42.98 & 38.38 & 2.8 & AHLT BNGL BTLS BYBT CMCY DGRL DGSU EZRM GNI HAMR HINS HRSN KIV KOTK KRLV MUSH \\
\hline 001115171348 & 42.72 & 38.42 & 2.9 & AHLT BNGL BTLS BYBT CMCY DGRL DGSU EZRM GNI HAMR HINS HRSN KIV KOTK KRLV MUSH \\
\hline 001115174113 & 42.96 & 38.18 & 3.3 & AHLT BNGL BTLS BYBT CMCY DGRL DGSU EZRM GNI HAMR HINS HRSN KIV KOTK KRLV MUSH \\
\hline 001115174413 & 42.83 & 38.28 & 3.5 & AHLT BNGL BTLS BYBT CMCY DGRL DGSU EZRM GNI HAMR HINS HRSN KIV KOTK KRLV MUSH \\
\hline 001115174941 & 42.86 & 38.26 & 2.8 & AHLT BNGL BTLS BYBT CMCY DGRL DGSU EZRM GNI HAMR HINS HRSN KIV KOTK KRLV MUSH \\
\hline 001115181610 & 43.05 & 38.31 & 3.7 & AHLT BNGL BTLS BYBT CMCY DGRL DGSU EZRM GNI HAMR HINS HRSN KIV KOTK KRLV MUSH \\
\hline 001115183211 & 43.01 & 38.71 & 5 & AHLT BNGL BTLS BYBT CMCY DGRL DGSU EZRM GNI HAMR HINS HRSN KIV KOTK KRLV MUSH \\
\hline 001115183858 & 42.83 & 38.41 & 3.4 & AHLT BNGL BTLS BYBT CMCY DGRL DGSU EZRM GNI HAMR HINS HRSN KIV KOTK KRLV MUSH \\
\hline 001115185607 & 42.86 & 38.2 & 2.8 & AHLT BNGL BTLS BYBT CMCY DGRL DGSU EZRM GNI HAMR HINS HRSN KIV KOTK KRLV MUSH \\
\hline 001115191026 & 42.86 & 38.3 & 2.8 & AHLT BNGL BTLS BYBT CMCY DGRL DGSU EZRM GNI HAMR HINS HRSN KIV KOTK KRLV MUSH \\
\hline 001115192629 & 42.69 & 38.45 & 2.8 & AHLT BNGL BTLS BYBT CMCY DGRL DGSU EZRM GNI HAMR HINS HRSN KIV KOTK KRLV MUSH \\
\hline 001115193452 & 43.04 & 38.08 & 3.7 & AHLT BNGL BTLS BYBT CMCY DGRL DGSU EZRM GNI HAMR HINS HRSN KIV KOTK KRLV MUSH \\
\hline 001115193726 & 42.86 & 38.37 & 2.8 & AHLT BNGL BTLS BYBT CMCY DGRL DGSU EZRM GNI HAMR HINS HRSN KIV KOTK KRLV MUSH \\
\hline 001115200834 & 42.93 & 38.09 & 3.2 & AHLT BNGL BTLS BYBT CMCY DGRL DGSU EZRM GNI HAMR HINS HRSN KIV KOTK KRLV MUSH \\
\hline 001115203055 & 42.87 & 38.25 & 3.4 & AHLT BNGL BTLS BYBT CMCY DGRL DGSU EZRM GNI HAMR HINS HRSN KIV KOTK KRLV MUSH \\
\hline 001115203433 & 42.78 & 38.37 & 3.1 & AHLT BNGL BTLS BYBT CMCY DGRL DGSU EZRM GNI HAMR HINS HRSN KIV KOTK KRLV MUSH \\
\hline 001115204303 & 42.96 & 38.05 & 3.3 & AHLT BNGL BTLS BYBT CMCY DGRL DGSU EZRM GNI HAMR HINS HRSN KIV KOTK KRLV MUSH \\
\hline 204447 & 42.74 & 8.29 & 2.8 & AHLT BNGL BTLS BYBT CMCY DGRL DGSU EZRM GNI HAMR HINS HRSN KIV KOTK KRLV MUSH \\
\hline 424 & 42.86 & 8.27 & 2.7 & J EZRM GNI HAMR HINS HRSN KIV KOTK \\
\hline 001 & 42.87 & 38.23 & 3.5 & MUSH \\
\hline 1749 & 43.27 & 8.0 & 3.8 & BYBT CMCY DGRL DGSU EZRM GNI HA \\
\hline 4109 & 42.84 & 38.45 & 2.7 & RSN KIV KOTK KRLV MUSH \\
\hline 24147 & 42.99 & 38.3 & 3 & AHLT BNGL BTLS BYBT CMCY DGRL DGSU EZRM GNI HAMR HINS HRSN KIV KOTK KRLV MUSH \\
\hline 001116021754 & 42.86 & 38.45 & 2.6 & AHLT BNGL BTLS BYBT CMCY DGRL DGSU EZRM GNI HAMR HINS HRSN KIV KOTK KRLV MUSH \\
\hline 25751 & 42.93 & 38.27 & 2.8 & AHLT BNGL BTLS BYBT CMCY DGRL DGSU EZRM GNI HAMR HINS HRSN KIV KOTK KRLV MUSH \\
\hline 1645 & 42.84 & 38.49 & 2.7 & AHLT BNGL BTLS BYBT CMCY DGRL DGSU EZRM GNI HAMR HINS HRSN KIV KOTK KRLV MUSH \\
\hline 1902 & 42.84 & 38.35 & 2.7 & AMR HINS HRSN KIV KOTK KRLV MUSH \\
\hline 525 & 42.9 & 38.22 & 2.8 & SU EZRM GNI HAMR HINS HRSN KIV KOTK KRLV MUSH \\
\hline 059 & 42.99 & 38.94 & 2.5 & USH \\
\hline 001 & 4 & 1 & 3 & AHLT BNGL BTLS BYBT CMCY DGRL DGSL \\
\hline 001 & 42.99 & 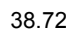 & 3.1 & AHLT BNGL BTLS BYBT CMCY DGRL DC \\
\hline 001 & 72 & 2 & 3 & AHLT BNGL BTLS BYBT CMCY DGRL DGSU EZRM GNI HAMR HINS HRSN KIV KOTK KRLV MUSH \\
\hline 001 & Th & 60 & 8 & U EZRM GNI HAMR HINS HRSN KIV KOTK KRLV MUSH \\
\hline 00 & 42.85 & 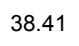 & 3.2 & 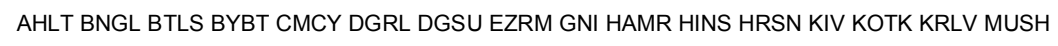 \\
\hline 00 & 42.85 & 38. & 2.9 & 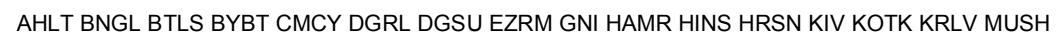 \\
\hline 4 & 42.93 & 38.4 & 3.5 & L \\
\hline oc & 43.13 & 38.65 & 2.8 & JSH \\
\hline 001116164539 & 42.99 & 38.3 & 3 & MUSH \\
\hline
\end{tabular}




\begin{tabular}{|c|c|c|c|c|}
\hline 001116175122 & 42.85 & 38.47 & 2.9 & AHLT BNGL BTLS BYBT CMCY DGRL DGSU EZRM GNI HAMR HINS HRSN KIV KOTK KRLV MUSH \\
\hline 001116190444 & 42.96 & 38.07 & 3 & AHLT BNGL BTLS BYBT CMCY DGRL DGSU EZRM GNI HAMR HINS HRSN KIV KOTK KRLV MUSH \\
\hline 001116191145 & 42.86 & 38.61 & 3 & AHLT BNGL BTLS BYBT CMCY DGRL DGSU EZRM GNI HAMR HINS HRSN KIV KOTK KRLV MUSH \\
\hline 001116200442 & 42.83 & 38.45 & 2.9 & AHLT BNGL BTLS BYBT CMCY DGRL DGSU EZRM GNI HAMR HINS HRSN KIV KOTK KRLV MUSH \\
\hline 001116211343 & 42.8 & 38.27 & 4 & PAR \\
\hline 001116211345 & 42.98 & 38.26 & 3.9 & AHLT BNGL BTLS BYBT CMCY DGRL DGSU EZRM GNI HAMR HINS HRSN KIV KOTK KRLV MUSH \\
\hline 001116231957 & 43.05 & 38.27 & 2.9 & AHLT BNGL BTLS BYBT CMCY DGRL DGSU EZRM GNI HAMR HINS HRSN KIV KOTK KRLV MUSH \\
\hline 001117002753 & 42.87 & 38.33 & 3.9 & PAR \\
\hline 001117002756 & 42.92 & 38.24 & 4.1 & AHLT BNGL BTLS BYBT CMCY DGRL DGSU EZRM GNI HAMR HINS HRSN KIV KOTK KRLV MUSH \\
\hline 001117083951 & 42.92 & 38.51 & 2.8 & AHLT BNGL BTLS BYBT CMCY DGRL DGSU EZRM GNI HAMR HINS HRSN KIV KOTK KRLV MUSH \\
\hline 001117093626 & 42.98 & 38.25 & 3.6 & AHLT BNGL BTLS BYBT CMCY DGRL DGSU EZRM GNI HAMR HINS HRSN KIV KOTK KRLV MUSH \\
\hline 001117133340 & 42.83 & 38.36 & 3.1 & AHLT BNGL BTLS BYBT CMCY DGRL DGSU EZRM GNI HAMR HINS HRSN KIV KOTK KRLV MUSH \\
\hline 001117144933 & 43.02 & 38.52 & 2.6 & AHLT BNGL BTLS BYBT CMCY DGRL DGSU EZRM GNI HAMR HINS HRSN KIV KOTK KRLV MUSH \\
\hline 001117212646 & 42.86 & 38.44 & 3.1 & AHLT BNGL BTLS BYBT CMCY DGRL DGSU EZRM GNI HAMR HINS HRSN KIV KOTK KRLV MUSH \\
\hline 001117235638 & 42.87 & 38.34 & 3 & AHLT BNGL BTLS BYBT CMCY DGRL DGSU EZRM GNI HAMR HINS HRSN KIV KOTK KRLV MUSH \\
\hline 001118011327 & 42.84 & 38.49 & 3.1 & AHLT BNGL BTLS BYBT CMCY DGRL DGSU EZRM GNI HAMR HINS HRSN KIV KOTK KRLV MUSH \\
\hline 001118124322 & 41.39 & 39.19 & 3 & AHLT BNGL BTLS BYBT CMCY DGRL DGSU EZRM GNI HAMR HINS HRSN KIV KOTK KRLV MUSH \\
\hline 001118125451 & 42.89 & 38.48 & 3.3 & AHLT BNGL BTLS BYBT CMCY DGRL DGSU EZRM GNI HAMR HINS HRSN KIV KOTK KRLV MUSH \\
\hline 001118154322 & 46.98 & 42.95 & 4 & AHLT BNGL BTLS BYBT CMCY DGRL DGSU EZRM GNI HAMR HINS HRSN KIV KOTK KRLV MUSH \\
\hline 001118180405 & 42.77 & 38.42 & 2.8 & AHLT BNGL BTLS BYBT CMCY DGRL DGSU EZRM GNI HAMR HINS HRSN KIV KOTK KRLV MUSH \\
\hline 001118212445 & 42.83 & 38.44 & 2.8 & AHLT BNGL BTLS BYBT CMCY DGRL DGSU EZRM GNI HAMR HINS HRSN KIV KOTK KRLV MUSH \\
\hline 001118233414 & 42.82 & 38.45 & 2.8 & AHLT BNGL BTLS BYBT CMCY DGRL DGSU EZRM GNI HAMR HINS HRSN KIV KOTK KRLV MUSH \\
\hline 001118235356 & 42.84 & 38.17 & 3 & AHLT BNGL BTLS BYBT CMCY DGRL DGSU EZRM GNI HAMR HINS HRSN KIV KOTK KRLV MUSH \\
\hline 001119000219 & 42.92 & 38.41 & 3.8 & AHLT BNGL BTLS BYBT CMCY DGRL DGSU EZRM GNI HAMR HINS HRSN KIV KOTK KRLV MUSH \\
\hline 001119002615 & 42.81 & 38.49 & 3.1 & AHLT BNGL BTLS BYBT CMCY DGRL DGSU EZRM GNI HAMR HINS HRSN KIV KOTK KRLV MUSH \\
\hline 001119015629 & 42.89 & 38.31 & 2.8 & AHLT BNGL BTLS BYBT CMCY DGRL DGSU EZRM GNI HAMR HINS HRSN KIV KOTK KRLV MUSH \\
\hline 001119031628 & 42.76 & 38.38 & 3 & AHLT BNGL BTLS BYBT CMCY DGRL DGSU EZRM GNI HAMR HINS HRSN KIV KOTK KRLV MUSH \\
\hline 001119033649 & 42.74 & 38.45 & 2.7 & AHLT BNGL BTLS BYBT CMCY DGRL DGSU EZRM GNI HAMR HINS HRSN KIV KOTK KRLV MUSH \\
\hline 001119044517 & 42.82 & 38.39 & 2.9 & AHLT BNGL BTLS BYBT CMCY DGRL DGSU EZRM GNI HAMR HINS HRSN KIV KOTK KRLV MUSH \\
\hline 001119075745 & 42.79 & 38.52 & 3.3 & AHLT BNGL BTLS BYBT CMCY DGRL DGSU EZRM GNI HAMR HINS HRSN KIV KOTK KRLV MUSF \\
\hline 001119164037 & 42.88 & 38.32 & 3.5 & AHLT BNGL BTLS BYBT CMCY DGRL DGSU EZRM GNI HAMR HINS HRSN KIV KOTK KRLV MUSH \\
\hline 001119164534 & 42.77 & 38.38 & 1 & AHLT BNGL BTLS BYBT CMCY DGRL DGSU EZRM GNI HAMR HINS HRSN KIV KOTK KRLV MUSH \\
\hline 172314 & 42.83 & 38.51 & 3.2 & AHLT BNGL BTLS BYBT CMCY DGRL DGSU EZRM GNI HAMR HINS HRSN KIV KOTK KRLV MUSH \\
\hline 0011 & 42.8 & 38.46 & 3 & AHLT BNGL BTLS BYBT CMCY DGRL DGSU EZRM GNI HAMR HINS HRSN KIV KOTK KRLV MUSH \\
\hline 001119214717 & 45.86 & 43.02 & 4.3 & DGRL DGSU EZRM GNI HAMR HINS HRSN KIV KOTK KRLV MUSH \\
\hline 001120043307 & 42.85 & 38.47 & 3 & AHLT BNGL BTLS BYBT CMCY DGRL DGSU EZRM GNI HAMR HINS HRSN KIV KOTK KRLV MUSH \\
\hline 001120060508 & 2.81 & 38.45 & 2 & GRL DGSU EZRM GNI HAMR HINS HRSN KIV KOTK KRLV MUSH \\
\hline 001120073443 & 42.83 & 38.56 & 1 & AHLT BNGL BTLS BYBT CMCY DGRL DGSU EZRM GNI HAMR HINS HRSN KIV KOTK KRLV MUSH \\
\hline 001120122317 & 42.86 & 38.46 & 3.1 & AHLT BNGL BTLS BYBT CMCY DGRL DGSU EZRM GNI HAMR HINS HRSN KIV KOTK KRLV MUSH \\
\hline 001120172627 & 42.92 & 8.35 & 3.1 & GGRL DGSU EZRM GNI HAMR HINS HRSN KIV KOTK KRLV MUSH \\
\hline 25741 & 42.82 & 8.5 & 5 & U EZRM GNI HAMR HINS HRSN KIV KOTK KRLV MUS \\
\hline 625 & 2.78 & 38.46 & 2.9 & AMR HINS HRSN KIV KOTK KRL \\
\hline 412 & 43 & 8. & 2.9 & HLT BNGL BTLS BYBT CMCY DGRL DGSU EZR \\
\hline 001121 & 42.77 & $3 \varepsilon$ & 3 & AHLT BNGL BTLS \\
\hline 0011 & 42. & 38. & 3 & NGI \\
\hline 0011 & 48.19 & 39.18 & 4.2 & AHLT BNGL BTLS BYBT CMCY DGRL DGSU EZRM GNI HAMR HINS HRSN KIV KOTK KRLV MUSH \\
\hline 001 & 42.97 & 38.32 & 3.8 & BYBT CMCY DGRL DGSU EZRM GNI HAMR HINS HRSN KIV KOTK KRLV MUSH \\
\hline 0011 & 42.8 & 38.42 & 3.6 & SSU EZRM GNI HAMR HINS HRSN KIV KOTK KRLV N \\
\hline 001 & 42.78 & 38.45 & 3 & - DILO D \\
\hline 001 & 2.71 & 38.37 & 3.2 & AHLT BNGL BTLS BYBT CMCY DGRL DGSU EZRM GNI HAMR HINS HRSN KIV KOTK KRLV MI \\
\hline $001125180 s$ & 49.94 & 40.22 & 6 & U EZRM GNI HAMR HINS HRSN KIV KOTK KRLV \\
\hline 001125181046 & 49.95 & 40.19 & 3 & U GNI HAMR HINS HRSN K \\
\hline
\end{tabular}




\begin{tabular}{|c|c|c|c|c|}
\hline 001125183555 & 50.16 & 39.93 & 3.7 & AHLT BNGL BTLS BYBT CMCY DGRL DGSU EZRM GNI HAMR HINS HRSN KIV KOTK KRLV MUSH \\
\hline 001125184747 & 49.93 & 40.25 & 4.3 & AHLT BNGL BTLS BYBT CMCY DGRL DGSU EZRM GNI HAMR HINS HRSN KIV KOTK KRLV MUSH \\
\hline 001125184913 & 50.12 & 40.09 & 4.2 & AHLT BNGL BTLS BYBT CMCY DGRL DGSU EZRM GNI HAMR HINS HRSN KIV KOTK KRLV MUSH \\
\hline 001125190502 & 49.3 & 40.94 & 3.9 & AHLT BNGL BTLS BYBT CMCY DGRL DGSU EZRM GNI HAMR HINS HRSN KIV KOTK KRLV MUSH \\
\hline 001125202533 & 50.14 & 40.14 & 3 & AHLT BNGL BTLS BYBT CMCY DGRL DGSU EZRM GNI HAMR HINS HRSN KIV KOTK KRLV MUSH \\
\hline 001125204614 & 49.95 & 40.2 & 3.9 & AHLT BNGL BTLS BYBT CMCY DGRL DGSU EZRM GNI HAMR HINS HRSN KIV KOTK KRLV MUSH \\
\hline 001125210105 & 50.09 & 40.12 & 3.8 & AHLT BNGL BTLS BYBT CMCY DGRL DGSU EZRM GNI HAMR HINS HRSN KIV KOTK KRLV MUSH \\
\hline 001125211847 & 49.62 & 40.13 & 3.9 & AHLT BNGL BTLS BYBT CMCY DGRL DGSU EZRM GNI HAMR HINS HRSN KIV KOTK KRLV MUSH \\
\hline 001125221504 & 49.95 & 40.22 & 3.9 & AHLT BNGL BTLS BYBT CMCY DGRL DGSU EZRM GNI HAMR HINS HRSN KIV KOTK KRLV MUSH \\
\hline 001126001820 & 50.12 & 40.08 & 3.9 & AHLT BNGL BTLS BYBT CMCY DGRL DGSU EZRM GNI HAMR HINS HRSN KIV KOTK KRLV MUSH \\
\hline 001126013053 & 50.24 & 40.24 & 3.6 & AHLT BNGL BTLS BYBT CMCY DGRL DGSU EZRM GNI HAMR HINS HRSN KIV KOTK KRLV MUSH \\
\hline 001126014134 & 50.13 & 40.06 & 3.9 & AHLT BNGL BTLS BYBT CMCY DGRL DGSU EZRM GNI HAMR HINS HRSN KIV KOTK KRLV MUSH \\
\hline 001126021921 & 50.13 & 40.2 & 3.9 & AHLT BNGL BTLS BYBT CMCY DGRL DGSU EZRM GNI HAMR HINS HRSN KIV KOTK KRLV MUSH \\
\hline 001126023255 & 50.15 & 40.12 & 3.8 & AHLT BNGL BTLS BYBT CMCY DGRL DGSU EZRM GNI HAMR HINS HRSN KIV KOTK KRLV MUSH \\
\hline 001126040023 & 49.97 & 40.23 & 4.2 & AHLT BNGL BTLS BYBT CMCY DGRL DGSU EZRM GNI HAMR HINS HRSN KIV KOTK KRLV MUSH \\
\hline 001126063636 & 50.16 & 40.03 & 3.7 & AHLT BNGL BTLS BYBT CMCY DGRL DGSU EZRM GNI HAMR HINS HRSN KIV KOTK KRLV MUSH \\
\hline 001126070818 & 46.13 & 43.32 & 4 & AHLT BNGL BTLS BYBT CMCY DGRL DGSU EZRM GNI HAMR HINS HRSN KIV KOTK KRLV MUSH \\
\hline 001126084758 & 50.12 & 39.95 & 4.1 & AHLT BNGL BTLS BYBT CMCY DGRL DGSU EZRM GNI HAMR HINS HRSN KIV KOTK KRLV MUSH \\
\hline 001126141651 & 50.08 & 40.03 & 3.9 & AHLT BNGL BTLS BYBT CMCY DGRL DGSU EZRM GNI HAMR HINS HRSN KIV KOTK KRLV MUSH \\
\hline 001126152545 & 50.15 & 40.13 & 3.2 & AHLT BNGL BTLS BYBT CMCY DGRL DGSU EZRM GNI HAMR HINS HRSN KIV KOTK KRLV MUSH \\
\hline 001126182306 & 50.04 & 40.32 & 3.2 & AHLT BNGL BTLS BYBT CMCY DGRL DGSU EZRM GNI HAMR HINS HRSN KIV KOTK KRLV MUSH \\
\hline 001126213710 & 50.16 & 40.18 & 4.2 & AHLT BNGL BTLS BYBT CMCY DGRL DGSU EZRM GNI HAMR HINS HRSN KIV KOTK KRLV MUSH \\
\hline 001127094109 & 50.22 & 40.16 & 3.6 & AHLT BNGL BTLS BYBT CMCY DGRL DGSU EZRM GNI HAMR HINS HRSN KIV KOTK KRLV MUSH \\
\hline 001127145021 & 42.86 & 38.46 & 2.9 & AHLT BNGL BTLS BYBT CMCY DGRL DGSU EZRM GNI HAMR HINS HRSN KIV KOTK KRLV MUSH \\
\hline 001127150535 & 42.87 & 38.38 & 3.3 & AHLT BNGL BTLS BYBT CMCY DGRL DGSU EZRM GNI HAMR HINS HRSN KIV KOTK KRLV MUSH \\
\hline 001127160450 & 50.21 & 40.11 & 3.9 & AHLT BNGL BTLS BYBT CMCY DGRL DGSU EZRM GNI HAMR HINS HRSN KIV KOTK KRLV MUSH \\
\hline 001127211654 & 49.85 & 40.24 & 3.8 & AHLT BNGL BTLS BYBT CMCY DGRL DGSU EZRM GNI HAMR HINS HRSN KIV KOTK KRLV MUSH \\
\hline 001128001432 & 50.15 & 40.19 & 3 & AHLT BNGL BTLS BYBT CMCY DGRL DGSU EZRM GNI HAMR HINS HRSN KIV KOTK KRLV MUSH \\
\hline 001128203726 & 50.01 & 40.16 & 4.7 & AHLT BNGL BTLS BYBT CMCY DGRL DGSU EZRM GNI HAMR HINS HRSN KIV KOTK KRLV MUSH \\
\hline 001129020708 & 50.52 & 40.01 & 2.5 & AHLT BNGL BTLS BYBT CMCY DGRL DGSU EZRM GNI HAMR HINS HRSN KIV KOTK KRLV MUSH \\
\hline 001129104503 & 50.09 & 39.97 & 4.1 & AHLT BNGL BTLS BYBT CMCY DGRL DGSU EZRM GNI HAMR HINS HRSN KIV KOTK KRLV MUSH \\
\hline 001130050951 & 50.07 & 40.12 & 4.1 & AHLT BNGL BTLS BYBT CMCY DGRL DGSU EZRM GNI HAMR HINS HRSN KIV KOTK KRLV MUSH \\
\hline 001130103007 & 42.89 & 38.05 & 3.7 & AHLT BNGL BTLS BYBT CMCY DGRL DGSU EZRM GNI HAMR HINS HRSN KIV KOTK KRLV MUSH \\
\hline 001201140827 & 50.03 & 40.27 & 3.8 & AHLT BNGL BTLS BYBT CMCY DGRL DGSU EZRM GNI HAMR HINS HRSN KIV KOTK KRLV MUSH \\
\hline 001201182831 & 42.75 & 38.4 & 3.1 & AHLT BNGL BTLS BYBT CMCY DGRL DGSU EZRM GNI HAMR HINS HRSN KIV KOTK KRLV MUSH \\
\hline 001202153523 & 47.37 & 42.46 & 4.6 & AHLT BNGL BTLS BYBT CMCY DGRL DGSU EZRM GNI HAMR HINS HRSN KIV KOTK KRLV MUSH \\
\hline 001202154300 & 50.2 & 40.14 & 3.8 & AHLT BNGL BTLS BYBT CMCY DGRL DGSU EZRM GNI HAMR HINS HRSN KIV KOTK KRLV MUSH \\
\hline 001202181106 & 49.89 & 40.33 & 3.6 & AHLT BNGL BTLS BYBT CMCY DGRL DGSU EZRM GNI HAMR HINS HRSN KIV KOTK KRLV MUSH \\
\hline 001202201941 & 42.82 & 38.45 & 3.2 & AHLT BNGL BTLS BYBT CMCY DGRL DGSU EZRM GNI HAMR HINS HRSN KIV KOTK KRLV MUSH \\
\hline 001202235433 & 47.32 & 42.29 & 4 & AHLT BNGL BTLS BYBT CMCY DGRL DGSU EZRM GNI HAMR HINS HRSN KIV KOTK KRLV MUSH \\
\hline 001203173755 & 47.49 & 42.28 & 4.1 & AHLT BNGL BTLS BYBT CMCY DGRL DGSU EZRM GNI HAMR HINS HRSN KIV KOTK KRLV MUSH \\
\hline 001204024501 & 42.97 & 38.97 & 3 & AHLT BNGL BTLS BYBT CMCY DGRL DGSU EZRM GNI H \\
\hline 001204034218 & 50.19 & 39.97 & 3.8 & AHLT BNGL BTLS BYBT CMCY DGRL DGSU EZRM GNI HAMR HINS HRSN KIV KOTK KRLV MUSH \\
\hline 001204083120 & 42.81 & 40.1 & 3.4 & AHLT BNGL BTLS BYBT CMCY DGRL DGSU EZRM GNI HAMR HINS HRSN KIV KOTK KRLV MUSH \\
\hline 001205012014 & 46.5 & 43.6 & 4 & AHLT BNGL BTLS BYBT CMCY DGRL DGSU EZRM GNI HAMR HINS HRSN KIV KOTK KRLV MUSH \\
\hline 001206044507 & 44.12 & 41.51 & 4 & AHLT AKS BNGL BTLS BYBT CMCY DGRL DGSU EZRM GNI HAMR HINS HRSN KIV KOTK KRLV MUSH \\
\hline 001206120415 & 50.74 & 40.1 & 4.1 & AHLT BNGL BTLS BYBT CMCY DGRL DGSU EZRM GNI HAMR HINS HRSN KIV KOTK KRLV MUSH \\
\hline 001208062248 & 44.82 & 43 & 4.4 & AHLT BNGL BTLS BYBT CMCY DGRL DGSU EZRM GNI HAMR HINS HRSN KIV KOTK KRLV MUSH \\
\hline 001209065036 & 44.22 & 42.53 & 4 & AHLT BNGL BTLS BYBT CMCY DGRL DGSU EZRM GNI HAMR HINS HRSN KIV KOTK KRLV MUSH \\
\hline 001209083705 & 43.94 & 42.38 & 3.7 & AHLT BNGL BTLS BYBT CMCY DGRL DGSU EZRM GNI HAMR HINS HRSN KIV KOTK KRLV MUSH \\
\hline 001209111954 & 42.81 & 38.49 & 2.9 & AHLT BNGL BTLS BYBT CMCY DGRL DGSU EZRM GNI HAMR HINS HRSN KIV KOTK KRLV MUSH \\
\hline 001209200352 & 50.48 & 40.42 & 3.1 & RM GNI HAMR HINS HRSN KIV KOTK KRLV MUSH \\
\hline
\end{tabular}




\begin{tabular}{|c|c|c|c|c|}
\hline 001210034241 & 42.76 & 38.74 & 3.2 & S BYBT CMCY DGRL DGSU EZRM GNI HAMR HINS HRSN KIV KOTK KRLV MUSH \\
\hline 001211174640 & 40.1 & 38.73 & 2.3 & AHLT BNGL BTLS BYBT CMCY DGRL DGSU EZRM GNI HAMR HINS HRSN KIV KOTK KRLV MUSH \\
\hline 001212022154 & 49.51 & 40.33 & 3.4 & AHLT BNGL BTLS BYBT CMCY DGRL DGSU EZRM GNI HAMR HINS HRSN KIV KOTK KRLV MUSH \\
\hline 001212164331 & 43.5 & 39.84 & 3.7 & AHLT BNGL BTLS BYBT CMCY DGRL DGSU EZRM GNI HAMR HINS HRSN KIV KOTK KRLV MUSH \\
\hline 001213195257 & 42.76 & 38.45 & 3.2 & AHLT BNGL BTLS BYBT CMCY DGRL DGSU EZRM GNI HAMR HINS HRSN KIV KOTK KRLV MUSH \\
\hline 001214154536 & 44.73 & 41.57 & 3.5 & AMB \\
\hline 001214154538 & 44.7 & 41.56 & 4.3 & AHLT BNGL BTLS BYBT CMCY DGRL DGSU EZRM GNI HAMR HINS HRSN KIV KOTK KRLV MUSH \\
\hline 001214154542 & -12345 & -12345 & 0 & STBO \\
\hline 001215134626 & 43.82 & 42.88 & 4 & AHLT BNGL BTLS BYBT CMCY DGRL DGSU EZRM GNI HAMR HINS HRSN KIV KOTK KRLV MUSH \\
\hline 001222225624 & 43.18 & 38.27 & 3.2 & AHLT BNGL BTLS CMCY DGRL DGSU EZRM GNI HAMR HINS HRSN KIV KOTK KRLV MUSH \\
\hline 001223082446 & 50.11 & 40.19 & 4 & AHLT BNGL BTLS CMCY DGRL DGSU EZRM GNI HAMR HINS HRSN KIV KOTK KRLV MUSH \\
\hline 001224113047 & 40.28 & 38.57 & 4.4 & AHLT BNGL BTLS CMCY DGRL DGSU EZRM GNI HAMR HINS HRSN KIV KOTK KRLV MUSH \\
\hline 001224163022 & 50.06 & 40.17 & 3.7 & AHLT BNGL BTLS CMCY DGRL DGSU EZRM GNI HAMR HINS HRSN KIV KOTK KRLV MUSH \\
\hline 001225030017 & 50.08 & 40.25 & 4 & AHLT BNGL BTLS CMCY DGRL DGSU EZRM GNI HAMR HINS HRSN KIV KOTK KRLV MUSH \\
\hline 001225175102 & 43.66 & 39.8 & 3.1 & AHLT BNGL BTLS CMCY DGRL DGSU EZRM GNI HAMR HINS HRSN KIV KOTK KRLV MUSH \\
\hline 001228020140 & 47.09 & 43.27 & 4.2 & AHLT BNGL BTLS CMCY DGRL DGSU EZRM GNI HAMR HINS HRSN KIV KOTK KRLV MUSH \\
\hline 001229003523 & 43.25 & 38.42 & 3.5 & AHLT BNGL BTLS CMCY DGRL DGSU EZRM GNI HINS HRSN KIV KOTK KRLV MUSH \\
\hline 001229021403 & 46.52 & 41.78 & 3.7 & AHLT BNGL BTLS CMCY DGRL DGSU EZRM GNI HAMR HINS HRSN KIV KOTK KRLV MUSH \\
\hline 001229171725 & 43.79 & 38.6 & 3.4 & AHLT BNGL BTLS CMCY DGRL DGSU EZRM GNI HAMR HINS HRSN KIV KOTK KRLV MUSH \\
\hline 001231011142 & 42.98 & 38.36 & 3.1 & AHLT BNGL BTLS CMCY DGRL DGSU EZRM GNI HAMR HINS HRSN KIV KOTK KRLV MUSH \\
\hline 001231120935 & 44.49 & 41.47 & 4.2 & AHLT BNGL BTLS CMCY DGRL DGSU EZRM GNI HAMR HINS HRSN KIV KOTK KRLV MUSH \\
\hline 001231185135 & 42.65 & 38.15 & 3.6 & AHLT BNGL BTLS CMCY DGRL DGSU EZRM GNI HAMR HINS HRSN KIV KOTK KRLV MUSH \\
\hline 001231190958 & 42.66 & 38.19 & 4 & AHLT BNGL BTLS CMCY DGRL DGSU EZRM GNI HAMR HINS HRSN KIV KOTK KRLV MUSH \\
\hline 010101165536 & 42.3 & 39.92 & 3.2 & AHLT BNGL BTLS CMCY DGRL DGSU EZRM GNI HAMR HINS HRSN KIV KOTK KRLV MUSH \\
\hline 010104003522 & 43.21 & 38.8 & 3.4 & AHLT BNGL BTLS CMCY DGRL DGSU EZRM GNI HAMR HINS HRSN KIV KOTK KRLV MUSH \\
\hline 010105000205 & 40.93 & 38.76 & 3.3 & AHLT BNGL BTLS CMCY DGRL DGSU EZRM GNI HAMR HINS HRSN KIV KOTK KRLV MUSH \\
\hline 010105000347 & 40.64 & 39.03 & 2.9 & AHLT BNGL BTLS CMCY DGRL DGSU EZRM GNI HAMR HINS HRSN KIV KOTK KRLV MUSH \\
\hline 010106232132 & 48.03 & 42.35 & 4.3 & AHLT BNGL BTLS CMCY DGRL DGSU EZRM GNI HAMR HINS HRSN KIV KOTK KRLV MUSH \\
\hline 010107204146 & 50.29 & 40.04 & 3.1 & AHLT BNGL BTLS CMCY DGRL DGSU EZRM GNI HAMR HINS HRSN KIV KOTK KRLV MUSH \\
\hline 010108031741 & 42.79 & 38.39 & 2.8 & AHLT BNGL BTLS DGRL DGSU EZRM HAMR HINS HRSN KIV KOTK KRLV MUSH \\
\hline 010108131650 & 42.82 & 43.98 & 3.7 & AHLT BNGL BTLS DGRL DGSU EZRM GNI HAMR HINS HRSN KIV KOTK KRLV MUSH \\
\hline 010109000449 & 47.62 & 41.6 & 4 & AHLT BNGL BTLS DGRL DGSU EZRM GNI HAMR HINS HRSN KIV KOTK KRLV MUSH \\
\hline 010110014948 & 42.8 & 38.44 & 2.9 & AHLT BNGL BTLS DGRL DGSU EZRM GNI HAMR HINS HRSN KIV KOTK KRLV MUSH \\
\hline 010110144218 & 42.15 & 38.43 & 2.8 & AHLT BNGL BTLS DGRL DGSU EZRM GNI HAMR HINS HRSN KIV KOTK KRLV MUSH \\
\hline 010111231023 & 41.51 & 38.31 & 2.6 & AHLT BNGL BTLS DGRL DGSU EZRM GNI HAMR HINS HRSN KIV KOTK KRLV MUSH \\
\hline 010112090528 & 42.24 & 38.86 & 3.1 & AHLT BNGL BTLS DGRL DGSU EZRM GNI HAMR HINS HRSN KIV KOTK KRLV MUSH \\
\hline 010112102015 & 41.63 & 38.07 & 3.1 & AHLT BNGL BTLS DGRL DGSU EZRM GNI HAMR HINS HRSN KIV KOTK KRLV MUSH \\
\hline 010112200204 & 42.81 & 39.74 & 2.6 & AHLT BNGL BTLS DGRL DGSU EZRM GNI HAMR HINS HRSN KIV KOTK KRLV MUSH \\
\hline 010114091020 & 43.03 & 39.16 & 3.8 & AHLT BNGL BTLS DGRL DGSU EZRM GNI HAMR HINS HRSN KIV KOTK KRLV MUSH \\
\hline 010115151406 & 45.23 & 38.33 & 3.6 & KAP \\
\hline 010117231430 & 43.46 & 39.88 & 3.2 & AHLT BNGL BTLS DGRL DGSU EZRM GNI HAMR HINS HRSN KIV KOTK MUSH \\
\hline 010121115153 & 49.99 & 40 & 4 & AHLT BNGL BTLS DGRL DGSU EZRM HAMR HINS HRSN KARS KIV KOTK KRLV MUSH \\
\hline 010121171027 & 50 & 40.62 & 4.3 & AHLT BNGL BTLS DGRL DGSU EZRM HAMR HINS HRSN KARS KIV KOTK KRLV MUSH \\
\hline 010122222650 & 41.59 & 38.47 & 2.3 & AHLT BNGL BTLS DGRL DGSU EZRM HAMR HINS HRSN KARS KIV KOTK KRLV MUSH \\
\hline 010123043814 & 42.79 & 38.44 & 2.8 & AHLT BNGL BTLS DGRL DGSU EZRM HAMR HINS HRSN KARS KIV KOTK KRLV MUSH \\
\hline 010123092753 & 46.61 & 40.16 & 3.5 & AHLT BNGL BTLS DGRL DGSU EZRM HAMR HINS HRSN KARS KIV KOTK KRLV MUSH VNN \\
\hline 010123092959 & 46.54 & 40.1 & 4 & AHLT BNGL BTLS DGRL DGSU EZRM HAMR HINS HRSN KARS KIV KOTK KRLV MUSH \\
\hline 010124231845 & 40.72 & 38.61 & 3.1 & AHLT BNGL BTLS DGRL DGSU EZRM HAMR HINS HRSN KARS KIV KOTK KRLV MUSH \\
\hline 010125041823 & 40.42 & 38.67 & 3.6 & AHLT BNGL BTLS DGRL DGSU EZRM HAMR HINS HRSN KARS KIV KOTK KRLV MUSH \\
\hline 521 & 43.39 & 39.98 & 3.3 & R HINS HRSN KARS KIV KOTK KRLV MUSH \\
\hline 007 & 46.42 & 40.69 & 4 & AHLT BNGL BTLS DGRL DGSU EZRM HAMR HINS HRSN KARS KIV KOTK KRLV MUSH \\
\hline 010128230239 & 42.99 & 38.51 & 3.5 & AHLT BNGL BTLS DGRL DGSU EZRM HAMR HINS HRSN KARS KIV KOTK KRLV \\
\hline
\end{tabular}




\begin{tabular}{|c|c|c|}
\hline 010129150307 & 40.69 & 38.57 \\
\hline 010129162507 & 49.6 & 39.33 \\
\hline 010129214958 & 46.6 & 42.9 \\
\hline 010130070123 & 43.29 & 38.24 \\
\hline 010131025444 & 44.14 & 41.94 \\
\hline 010131120211 & 47.85 & 43.11 \\
\hline 010201024253 & 44.6 & 38.66 \\
\hline 010203005104 & 41.13 & 39.21 \\
\hline 010203161638 & 45.65 & 41.41 \\
\hline 010204065245 & 43.93 & 42.41 \\
\hline 010205050054 & 50.3 & 39.94 \\
\hline 010205072743 & 43.64 & 42.28 \\
\hline 010205175805 & 46.16 & 43.51 \\
\hline 010205190221 & 48.6 & 38.61 \\
\hline 010206003051 & 44.67 & 43.27 \\
\hline 010210110219 & 50.84 & 40.1 \\
\hline 010210204219 & 43.57 & 38.63 \\
\hline 010210204225 & 43.55 & 38.8 \\
\hline 010211192219 & 50.69 & 39.92 \\
\hline 010215022352 & 40.54 & 39.05 \\
\hline 010216101755 & 42.03 & 44.03 \\
\hline 010216142935 & 50.04 & 38.42 \\
\hline 010217144113 & 43.22 & 38.39 \\
\hline 010221221323 & 42.52 & 38.71 \\
\hline 010222014105 & 47.35 & 43.17 \\
\hline 010222014641 & 47.39 & 43.2 \\
\hline 010225164602 & 50.13 & 39.93 \\
\hline 010227055454 & 46.69 & 42.83 \\
\hline 010227061209 & 40.02 & 39 \\
\hline 010227214411 & 41.39 & 38.56 \\
\hline 010311012248 & 40.58 & 38.58 \\
\hline 010311105705 & 46.33 & 41.74 \\
\hline 010313012714 & 40.13 & 38.82 \\
\hline 010314113800 & 41.84 & 38.98 \\
\hline 010315165310 & 40.11 & 39.38 \\
\hline 010318005209 & 45.06 & 42.2 \\
\hline 010325095601 & 40.43 & 39.11 \\
\hline 010329200259 & 40.62 & 38.65 \\
\hline 010331172017 & 41.62 & 39.16 \\
\hline 010401203722 & 40.02 & 39.5 \\
\hline 010403213002 & 40.44 & 39.03 \\
\hline 010410013159 & 49.33 & 39.79 \\
\hline 010410022014 & 46.55 & 42.13 \\
\hline 010411040531 & 43.46 & 42.49 \\
\hline 010413103327 & 42.66 & 44.19 \\
\hline 010417095338 & 48.97 & 41.74 \\
\hline 010419090256 & 46.81 & 42.68 \\
\hline 010420143112 & 42.88 & 39.7 \\
\hline 010420214752 & 44.34 & 39.25 \\
\hline 010420223934 & 44.93 & 43.44 \\
\hline 010421055757 & 49.9 & 40.22 \\
\hline 010421144850 & 46.04 & 43.2 \\
\hline
\end{tabular}

3.1
AHLT BNGL BTLS DGRL DGSU EZRM HAMR HINS HRSN KARS KIV KOTK KRLV MUSH AHLT BNGL BTLS DGRL DGSU EZRM HAMR HINS HRSN KARS KIV KOTK KRLV MUSH AHLT BNGL BTLS DGRL DGSU EZRM HAMR HINS HRSN KARS KIV KOTK KRLV MUSH AHLT BNGL BTLS DGRL DGSU EZRM HAMR HINS HRSN KARS KIV KOTK KRLV AHLT BNGL BTLS DGRL DGSU EZRM GNI HAMR HINS HRSN KARS KIV KOTK KRLV MUSH AHLT BNGL BTLS DGRL DGSU EZRM GNI HAMR HINS HRSN KARS KIV KOTK KRLV MUSH AHLT BNGL BTLS DGRL DGSU EZRM GNI HAMR HINS HRSN KARS KIV KOTK KRLV MUSH AHLT BNGL BTLS DGRL DGSU EZRM GNI HAMR HINS HRSN KARS KIV KOTK KRLV MUSH AHLT BNGL BTLS DGRL DGSU EZRM GNI HAMR HINS HRSN KARS KIV KOTK KRLV MUSH AHLT BNGL BTLS DGRL DGSU EZRM GNI HAMR HINS HRSN KARS KIV KOTK KRLV MUSH AHLT BNGL BTLS DGRL DGSU EZRM GNI HAMR HINS HRSN KARS KIV KOTK MUSH AHLT BNGL BTLS DGRL DGSU EZRM GNI HAMR HINS HRSN KARS KIV KOTK KRLV MUSH AHLT BNGL BTLS DGRL DGSU EZRM GNI HAMR HINS HRSN KARS KIV KOTK KRLV MUSH AHLT BNGL BTLS DGRL DGSU EZRM GNI HAMR HINS HRSN KARS KIV KOTK KRLV MUSH AHLT BNGL BTLS DGRL DGSU EZRM GNI HAMR HINS HRSN KARS KIV KOTK KRLV MUSH AHLT BNGL BTLS DGRL DGSU EZRM GNI HAMR HINS HRSN KARS KIV KOTK KRLV MUSH PAR

AHLT BNGL BTLS DGRL DGSU EZRM GNI HAMR HINS HRSN KARS KIV KOTK KRLV MUSH AHLT BNGL BTLS DGRL DGSU EZRM GNI HAMR HINS HRSN KARS KIV KOTK KRLV MUSH AHLT BNGL BTLS DGRL DGSU EZRM GNI HAMR HINS HRSN KARS KIV KOTK KRLV MUSH AHLT BNGL BTLS DGRL DGSU EZRM GNI HAMR HINS HRSN KARS KIV KOTK KRLV MUSH AHLT BNGL BTLS DGRL DGSU EZRM GNI HAMR HINS HRSN KARS KIV KOTK KRLV MUSH AHLT BNGL BTLS DGRL DGSU EZRM GNI HAMR HINS HRSN KARS KIV KOTK KRLV MUSH AHLT BNGL BTLS DGRL DGSU EZRM GNI HAMR HINS HRSN KARS KIV KOTK KRLV MUSH AHLT BNGL BTLS DGRL DGSU EZRM GNI HAMR HINS HRSN KARS KIV KOTK KRLV MUSH AHLT BNGL BTLS DGRL DGSU EZRM GNI HAMR HINS HRSN KARS KIV KOTK KRLV MUSH AHLT BNGL BTLS DGRL DGSU EZRM GNI HAMR HINS HRSN KARS KIV KOTK KRLV MUSH AHLT BNGL BTLS DGRL DGSU EZRM GNI HAMR HINS HRSN KARS KIV KOTK KRLV MUSH AHLT BNGL BTLS DGRL DGSU EZRM GNI HAMR HINS HRSN KARS KIV KOTK KRLV MUSH AHLT BNGL BTLS DGRL DGSU EZRM GNI HAMR HINS HRSN KARS KIV KOTK KRLV MUSH AHLT BNGL BTLS DGRL DGSU EZRM GNI HAMR HINS HRSN KIV KOTK KRLV MUSH AHLT BNGL BTLS DGRL DGSU EZRM GNI HAMR HINS HRSN KIV KOTK KRLV MUSH AHLT BNGL BTLS DGRL DGSU EZRM GNI HAMR HINS HRSN KIV KOTK KRLV MUSH AHLT BNGL BTLS DGRL DGSU EZRM GNI HAMR HINS HRSN KIV KOTK KRLV MUSH AHLT BNGL BTLS BYBT DGRL DGSU EZRM GNI HAMR HINS HRSN KIV KOTK KRLV MUSH AHLT BNGL BTLS BYBT DGRL DGSU EZRM GNI HAMR HINS HRSN KIV KOTK KRLV MUSH AHLT BNGL BTLS BYBT DGRL DGSU EZRM GNI HAMR HINS HRSN KIV KOTK KRLV MUSH AHLT BNGL BTLS BYBT DGRL DGSU EZRM GNI HAMR HINS HRSN KIV KOTK KRLV AHLT BNGL BTLS BYBT DGRL DGSU EZRM GNI HAMR HINS HRSN KIV KOTK KRLV AHLT BNGL BTLS BYBT DGRL DGSU EZRM GNI HAMR HINS HRSN KIV KOTK KRLV AHLT BNGL BTLS BYBT DGRL DGSU EZRM GNI HAMR HINS HRSN KIV KOTK AHLT BNGL BTLS BYBT DGRL DGSU EZRM GNI HAMR HINS HRSN KIV KOTK AHLT BNGL BTLS BYBT DGRL DGSU EZRM GNI HAMR HINS HRSN KIV KOTK AHLT BNGL BTLS BYBT DGRL DGSU EZRM GNI HAMR HINS HRSN KIV KOTK SILN AHLT BNGL BTLS BYBT BYKN DGRL DGSU EZRM GNI HAMR HINS HRSN KIV KOTK KRLV SILN AHLT BNGL BTLS BYBT BYKN DGRL DGSU EZRM GNI HAMR HINS HRSN KARS KIV KOTK KRLV MUSH SILN AHLT BNGL BTLS BYBT BYKN CMCY DGRL EZRM GNI HAMR HINS HRSN KARS KIV KOTK KRLV MUSH SILN AHLT BNGL BTLS BYBT BYKN DGRL EZRM GNI HAMR HINS HRSN KARS KIV KOTK KRLV MUSH SILN AHLT BNGL BTLS BYBT BYKN DGRL EZRM GNI HAMR HINS HRSN KARS KIV KOTK MUSH SILN AHLT BNGL BTLS BYBT BYKN DGRL EZRM GNI HAMR HINS HRSN KARS KIV KOTK MUSH SILN AHLT BNGL BTLS BYBT BYKN DGRL EZRM GNI HAMR HINS HRSN KARS KIV KOTK MUSH SILN AHLT BNGL BTLS BYBT BYKN DGRL EZRM GNI HAMR HINS HRSN KARS KIV KOTK KRLV MUSH SILN 


\begin{tabular}{|c|c|c|c|c|}
\hline 010422163022 & 46.81 & 43.19 & 4.2 & BT BYKN DGRL EZRM GNI HAMR HINS HRSN KARS KIV KOTK KRLV MUSH SILN \\
\hline 010423040919 & 45.72 & 43.21 & 4.4 & AHLT BNGL BTLS BYBT BYKN DGRL EZRM GNI HAMR HINS HRSN KARS KIV KOTK MUSH SILN \\
\hline 010428035955 & 40.44 & 38.72 & 3.5 & AHLT BNGL BTLS BYBT BYKN DGRL EZRM GNI HAMR HINS HRSN KARS KIV KOTK MUSH SILN \\
\hline 010428225251 & 50.06 & 40.23 & 3.6 & AHLT BNGL BTLS BYBT BYKN DGRL EZRM GNI HAMR HINS HRSN KARS KIV KOTK KRLV MUSH SILN \\
\hline 010429163409 & 48.52 & 40.04 & 4.9 & AHLT BNGL BTLS BYBT BYKN DGRL EZRM GNI HAMR HINS HRSN KARS KIV KOTK KRLV MUSH SILN \\
\hline 010507195148 & 45.01 & 43.9 & 3.5 & AHLT BNGL BTLS BYBT BYKN DGRL EZRM GNI HAMR HINS HRSN KARS KIV KOTK MUSH SILN \\
\hline 010507203458 & 45.11 & 43.8 & 4.1 & AHLT BNGL BTLS BYBT BYKN DGRL EZRM GNI HAMR HINS HRSN KARS KIV KOTK MUSH SILN \\
\hline 010507203916 & 45.09 & 43.79 & 4.3 & AHLT BNGL BTLS BYBT BYKN DGRL EZRM GNI HAMR HINS HRSN KARS KIV KOTK MUSH SILN \\
\hline 010507220724 & 44.96 & 43.53 & 3.8 & AHLT BNGL BTLS BYBT BYKN DGRL EZRM GNI HAMR HINS HRSN KARS KIV KOTK MUSH SILN \\
\hline 010507224821 & 45.01 & 43.87 & 3.4 & AHLT BNGL BTLS BYBT BYKN DGRL EZRM GNI HAMR HINS HRSN KARS KIV KOTK MUSH SILN \\
\hline 010507225115 & 44.95 & 43.66 & 3.9 & AHLT BNGL BTLS BYBT BYKN DGRL EZRM GNI HAMR HINS HRSN KARS KIV KOTK MUSH SILN \\
\hline 010507234345 & 45.02 & 43.85 & 3.7 & AHLT BNGL BTLS BYBT BYKN DGRL EZRM GNI HAMR HINS HRSN KARS KIV KOTK MUSH SILN \\
\hline 010508002010 & 44.97 & 43.66 & 3.8 & AHLT BNGL BTLS BYBT BYKN DGRL EZRM GNI HAMR HINS HRSN KARS KIV KOTK MUSH SILN \\
\hline 010508033925 & 41.26 & 39.21 & 3.5 & AHLT BNGL BTLS BYBT BYKN DGRL EZRM GNI HAMR HINS HRSN KARS KIV KOTK MUSH SILN \\
\hline 010510025639 & 45.92 & 41.84 & 4 & AHLT BNGL BTLS BYBT BYKN DGRL EZRM GNI HAMR HINS HRSN KARS KIV KOTK MUSH SILN \\
\hline 010511123033 & 40.04 & 38.68 & 4.2 & AHLT BNGL BTLS BYBT BYKN DGRL EZRM GNI HAMR HINS HRSN KARS KIV KOTK KRLV MUSH SILN \\
\hline 010513021901 & 44.33 & 43.82 & 3.5 & AHLT BNGL BTLS BYBT BYKN DGRL EZRM GNI HAMR HINS HRSN KARS KIV KOTK SILN \\
\hline 010517015829 & 42.08 & 43.54 & 3.5 & AHLT BNGL BTLS BYBT BYKN DGRL EZRM GNI HAMR HINS HRSN KARS KIV KOTK SILN \\
\hline 010518202922 & 41.55 & 38.34 & 2.6 & AHLT BNGL BTLS BYBT BYKN DGRL EZRM GNI HAMR HINS HRSN KARS KIV KOTK SILN \\
\hline 010520202929 & 49.79 & 40.47 & 4.3 & BNGL BTLS BYBT BYKN DGRL EZRM GNI HAMR HINS HRSN KARS KIV KOTK SILN \\
\hline 010520214705 & 42.89 & 38.28 & 3.1 & BNGL BTLS BYBT BYKN DGRL EZRM GNI HAMR HINS HRSN KARS KIV KOTK SILN \\
\hline 010521004912 & 49.33 & 39.85 & 3.2 & BNGL BTLS BYBT BYKN DGRL EZRM GNI HAMR HINS HRSN KARS KIV KOTK SILN \\
\hline 010524171532 & 41.39 & 39.83 & 3.4 & BNGL BTLS BYBT BYKN DGRL EZRM GNI HAMR HINS HRSN KARS KIV KOTK KRLV SILN \\
\hline 010525013652 & 40.44 & 39.02 & 2 & BNGL BTLS BYBT BYKN DGRL EZRM GNI HAMR HINS HRSN KARS KIV KOTK SILN \\
\hline 010526175703 & 43.11 & 38.02 & 3.1 & BNGL BTLS BYBT BYKN DGRL EZRM GNI HAMR HINS HRSN KARS KIV KOTK KRLV SILN \\
\hline 010528075050 & 45.79 & 43.16 & 4.2 & BNGL BTLS BYBT BYKN DGRL EZRM GNI HAMR HINS HRSN KARS KIV KOTK SILN \\
\hline 010529131429 & 41.67 & 39.84 & 4.6 & BNGL BTLS BYBT BYKN DGRL EZRM GNI HAMR HINS HRSN KARS KIV KOTK KRLV SILN \\
\hline 010529131433 & 41.55 & 39.85 & 4.6 & ERZ ERZ ERZ \\
\hline 010529141550 & 41.81 & 39.8 & 4.5 & ERZ ERZ ERZ \\
\hline 010529224540 & 43.23 & 39.04 & 3.4 & BNGL BTLS BYBT BYKN DGRL EZRM GNI HAMR HINS HRSN KARS KIV KOTK SILN \\
\hline 010530222351 & 41.81 & 39.8 & 3.1 & BNGL BTLS BYBT BYKN DGRL EZRM GNI HAMR HINS HRSN KARS KIV KOTK KRLV SILN \\
\hline 010531071453 & 45.69 & 43 & 4.3 & BNGL BTLS BYBT BYKN DGRL EZRM GNI HAMR HINS HRSN KARS KIV KOTK SILN \\
\hline 010601010020 & 40.08 & 38.26 & 3 & BNGL BTLS BYBT BYKN DGRL EZRM GNI HAMR HINS HRSN KARS KIV KOTK SILN \\
\hline 010601042838 & 49.96 & 40.99 & 4.2 & BNGL BTLS BYBT BYKN DGRL EZRM GNI HAMR HINS HRSN KARS KIV KOTK SILN \\
\hline 010601110042 & 40 & 38.89 & 3.2 & BNGL BTLS BYBT BYKN DGRL EZRM GNI HAMR HINS HRSN KARS KIV KOTK KRLV SILN \\
\hline 010601141451 & 40.35 & 39.08 & 3.4 & BNGL BTLS BYBT BYKN DGRL EZRM GNI HAMR HINS HRSN KARS KIV KOTK KRLV SILN \\
\hline 010604184746 & 41.64 & 39.95 & 3.3 & BNGL BTLS BYBT BYKN DGRL EZRM GNI HAMR HINS HRSN KARS KIV KOTK KRLV SILN \\
\hline 010605144255 & 48.86 & 42.77 & 4.1 & BNGL BTLS BYBT BYKN DGRL EZRM GNI HAMR HINS HRSN KARS KIV KOTK KRLV SILN \\
\hline 010606094159 & 40.78 & 38.61 & 2.9 & BNGL BTLS BYBT BYKN DGRL EZRM GNI HAMR HINS HRSN KARS KIV KOTK KRLV SILN \\
\hline 010606122652 & 40.7 & 38.57 & 2.7 & BNGL BTLS BYBT BYKN DGRL EZRM GNI HAMR HINS HRSN KARS KIV KOTK KRLV SILN \\
\hline 010606155147 & 40.01 & 38.89 & 3.6 & BNGL BTLS BYBT BYKN DGRL EZRM GNI HAMR HINS HRSN KARS KIV KOTK KRLV SILN \\
\hline 010607220831 & 42.82 & 38.24 & 3 & BNGL BTLS BYBT BYKN DGRL EZRM GNI HAMR HINS HRSN KARS KIV KOTK SILN \\
\hline 010607230448 & 40.08 & 38.83 & 3.3 & BNGL BTLS BYBT BYKN DGRL EZRM GNI HAMR HINS HRSN KARS KIV KOTK SILN \\
\hline 010607231339 & 42.88 & 38.07 & 3 & BNGL BTLS BYBT BYKN DGRL EZRM GNI HAMR HINS HRSN KARS KIV KOTK SILN \\
\hline 010608105027 & 48.35 & 41.98 & 4.4 & BNGL BTLS BYBT BYKN DGRL EZRM GNI HAMR HINS HRSN KARS KIV KOTK KRLV SILN \\
\hline 010608152553 & 42.83 & 38.54 & 3.3 & BNGL BTLS BYBT BYKN DGRL EZRM GNI HAMR HINS HRSN KARS KIV KOTK KRLV SILN \\
\hline 010613053237 & 40.58 & 38.61 & 3.3 & BNGL BTLS BYBT BYKN DGRL EZRM GNI HAMR HINS HRSN KARS KIV KOTK SILN \\
\hline 010615074921 & 42.48 & 38.8 & 3.8 & BNGL BTLS BYBT BYKN DGRL EZRM GNI HAMR HINS HRSN KARS KIV KOTK SILN \\
\hline 010617005420 & 40.53 & 39.3 & 3.1 & BNGL BTLS BYBT BYKN DGRL EZRM GNI HAMR HINS HRSN KARS KIV KOTK SILN \\
\hline 010617010146 & 42.74 & 38.43 & 2.9 & BNGL BTLS BYBT BYKN DGRL EZRM GNI HAMR HINS HRSN KARS KIV KOTK SILN \\
\hline 010617112215 & 45.81 & 39.04 & 4.4 & BNGL BTLS BYBT BYKN DGRL EZRM GNI HAMR HINS HRSN KARS KIV KOTK KRLV SILN \\
\hline 010617182108 & 41.33 & 40.09 & 3.6 & BNGL BTLS BYBT BYKN DGRL EZRM GNI HAMR HINS HRSN KARS KIV KOTK SILN \\
\hline
\end{tabular}




\begin{tabular}{|c|c|c|c|c|}
\hline 010618021255 & 46.74 & 43.53 & 4 & BNGL BTLS BYBT BYKN DGRL EZRM GNI HAMR HINS HRSN KARS KIV KOTK SILN \\
\hline 010620095432 & 42.97 & 38.57 & 2.9 & BNGL BTLS BYBT BYKN DGRL EZRM GNI HAMR HINS HRSN KARS KIV KOTK KRLV SILN \\
\hline 010620204200 & 47.84 & 38.03 & 3.3 & BNGL BTLS BYBT BYKN CMCY DGRL EZRM GNI HAMR HINS HRSN KARS KIV KOTK KRLV SILN \\
\hline 010621220751 & 42.32 & 38.97 & 3.6 & BNGL BTLS BYBT BYKN CMCY DGRL EZRM GNI HAMR HINS HRSN KARS KIV KOTK SILN \\
\hline 010626192841 & 41.69 & 39.9 & 4.3 & BNGL BTLS BYBT BYKN CMCY DGRL EZRM GNI HAMR HINS HRSN KARS KIV KOTK KRLV SILN \\
\hline 010627070729 & 42.02 & 39.8 & 3.3 & BNGL BTLS BYBT BYKN CMCY DGRL EZRM GNI HAMR HINS HRSN KARS KIV KOTK SILN \\
\hline 010627215829 & 47.88 & 42.1 & 4.6 & BNGL BTLS BYBT BYKN CMCY DGRL EZRM GNI HAMR HINS HRSN KARS KIV KOTK SILN \\
\hline 010701082430 & 47.95 & 41.33 & 4.2 & BNGL BTLS BYBT BYKN CMCY DGRL EZRM GNI HAMR HINS HRSN KARS KIV KOTK SILN \\
\hline 010703080724 & 43.39 & 39.13 & 3.3 & BNGL BTLS BYBT BYKN CMCY DGRL EZRM GNI HAMR HINS HRSN KARS KIV KOTK SILN \\
\hline 010704012849 & 47 & 42.97 & 4.9 & BNGL BTLS BYBT BYKN CMCY DGRL EZRM GNI HAMR HINS HRSN KARS KIV KOTK SILN \\
\hline 010706095643 & 46.12 & 41.67 & 4 & BNGL BTLS BYBT BYKN DGRL EZRM GNI HAMR HINS HRSN KARS KIV KOTK KRLV SILN \\
\hline 010708062623 & 40.47 & 39.46 & 3.5 & BNGL BTLS BYBT BYKN DGRL EZRM GNI HAMR HINS HRSN KARS KIV KOTK SILN \\
\hline 010708170342 & 46.15 & 42.07 & 4.6 & BNGL BTLS BYBT BYKN DGRL EZRM GNI HAMR HINS HRSN KARS KIV KOTK KRLV SILN \\
\hline 010708170436 & 46.17 & 41.62 & 3.7 & HAR KAP \\
\hline 010709053705 & 45.73 & 43.14 & 3.9 & BNGL BTLS BYBT BYKN DGRL EZRM GNI HAMR HINS HRSN KARS KIV KOTK SILN \\
\hline 010709184921 & 40.27 & 39.33 & 3.5 & BNGL BYBT BYKN DGRL EZRM GNI HAMR HINS HRSN KARS KIV KOTK KRLV SILN \\
\hline 010710005520 & 41.18 & 39.77 & 3.2 & BNGL BYBT BYKN DGRL EZRM GNI HAMR HINS HRSN KARS KIV KOTK SILN \\
\hline 010710010705 & 41.18 & 39.8 & 2.8 & BNGL BYBT BYKN DGRL EZRM GNI HAMR HINS HRSN KARS KIV KOTK SILN \\
\hline 010710111333 & 45.73 & 42.38 & 3.9 & KAP \\
\hline 010710111334 & 45.42 & 42.01 & 4.5 & BNGL BYBT BYKN DGRL EZRM GNI HAMR HINS HRSN KARS KIV KOTK KRLV SILN \\
\hline 010710152448 & 40.02 & 38.81 & 2.7 & BNGL BYBT BYKN DGRL EZRM HAMR HINS HRSN KARS KIV KOTK KRLV SILN \\
\hline 010710153847 & 41.57 & 39.97 & 3.4 & BNGL BYBT BYKN DGRL EZRM GNI HAMR HINS HRSN KARS KIV KOTK KRLV SILN \\
\hline 010710161433 & 41.47 & 39.98 & 3 & BNGL BYBT BYKN DGRL EZRM HAMR HINS HRSN KARS KIV KOTK KRLV SILN \\
\hline 010710173617 & 41.63 & 39.94 & 3.1 & BNGL BYBT BYKN DGRL EZRM GNI HAMR HINS HRSN KARS KIV KOTK KRLV SILN \\
\hline 010710214204 & 41.62 & 39.827 & 5.4 & ERZ ERZ ERZ \\
\hline 010710215656 & 41.57 & 39.88 & 3.4 & BNGL BYBT BYKN DGRL EZRM GNI HAMR HINS HRSN KARS KIV KOTK SILN \\
\hline 010710230656 & 41.61 & 39.85 & 2.8 & BNGL BYBT BYKN DGRL EZRM GNI HAMR HINS HRSN KARS KIV KOTK SILN \\
\hline 010710234621 & 41.54 & 39.94 & 3.1 & BNGL BYBT BYKN DGRL EZRM GNI HAMR HINS HRSN KARS KIV KOTK SILN \\
\hline 010710235247 & 41.45 & 39.94 & 3.2 & BNGL BYBT BYKN DGRL EZRM GNI HAMR HINS HRSN KARS KIV KOTK SILN \\
\hline 010711230659 & 41.61 & 39.85 & 3 & BNGL BYBT BYKN DGRL EZRM GNI HAMR HINS HRSN KARS KIV KOTK SILN \\
\hline 010711232225 & 41.03 & 39.74 & 2.8 & BNGL BYBT BYKN DGRL EZRM GNI HAMR HINS HRSN KARS KIV KOTK SILN \\
\hline 010711234620 & 41.52 & 39.94 & 3 & BNGL BYBT BYKN DGRL EZRM GNI HAMR HINS HRSN KARS KIV KOTK SILN \\
\hline 010711235248 & 41.52 & 39.9 & 3 & BNGL BYBT BYKN DGRL EZRM GNI HAMR HINS HRSN KARS KIV KOTK SILN \\
\hline 010712004625 & 41.54 & 39.12 & 3.1 & BNGL BYBT BYKN DGRL EZRM GNI HAMR HINS HRSN KARS KIV KOTK SILN \\
\hline 010712021449 & 46.2 & 43.11 & 4.4 & BNGL BYBT BYKN DGRL EZRM GNI HAMR HINS HRSN KARS KIV KOTK SILN \\
\hline 010713122206 & 48.97 & 40.72 & 3.8 & BNGL BYBT BYKN DGRL EZRM GNI HAMR HINS HRSN KARS KIV KOTK KRLV SILN \\
\hline 010713183132 & 44.34 & 41.37 & 4 & BNGL BYBT BYKN DGRL EZRM GNI HAMR HINS HRSN KARS KIV KOTK SILN \\
\hline 010714103726 & 40.9 & 39.5 & 3.5 & BNGL BYBT BYKN DGRL EZRM GNI HAMR HINS HRSN KARS KIV KOTK KRLV SILN \\
\hline 010718102346 & 41.66 & 44.04 & 4 & BNGL BYBT BYKN DGRL EZRM GNI HAMR HINS HRSN KARS KIV KOTK KRLV SILN \\
\hline 010722120959 & 43.89 & 43.16 & 4 & BNGL BYBT BYKN DGRL EZRM GNI HAMR HINS HRSN KARS KIV KOTK KRLV SILN \\
\hline 010722133422 & 43.68 & 42.37 & 4 & BNGL BYBT BYKN DGRL EZRM GNI HAMR HINS HRSN KARS KIV KOTK KRLV SILN \\
\hline 010722222016 & 43.17 & 38.77 & 2.9 & BNGL BYBT BYKN DGRL EZRM GNI HAMR HINS HRSN KARS KIV KOTK SILN \\
\hline 010723205722 & 40.33 & 38.49 & 2.7 & BNGL BYBT BYKN DGRL EZRM GNI HAMR HINS HRSN KARS KIV KOTK SILN \\
\hline 010725011443 & 40.39 & 38.52 & 2.9 & BNGL BYBT BYKN DGRL EZRM GNI HAMR HINS HRSN KARS KIV KOTK SILN \\
\hline 010725132815 & 43.12 & 44.05 & 4 & BNGL BYBT BYKN DGRL EZRM GNI HAMR HINS HRSN KARS KIV KOTK KRLV SILN \\
\hline 010727180719 & 40.43 & 38.79 & 2.9 & BNGL BYBT BYKN DGRL EZRM GNI HAMR HINS HRSN KARS KIV KOTK SILN \\
\hline 010729013621 & 47.19 & 40.57 & 4.3 & BNGL BYBT BYKN DGRL EZRM GNI HAMR HINS HRSN KARS KIV KOTK SILN \\
\hline 010729190118 & 48.38 & 42.04 & 4.1 & BNGL BYBT BYKN DGRL EZRM GNI HAMR HINS HRSN KARS KIV KOTK SILN \\
\hline 010801095903 & 40.68 & 39.18 & 3.1 & BNGL BYBT BYKN GNI HAMR KIV KRLV SILN \\
\hline 010804031010 & 40.55 & 39.19 & 2.1 & BYKN GNI SILN \\
\hline 010807033854 & 48.41 & 42.19 & 4 & GNI KIV \\
\hline 010811034528 & 48.38 & 42.22 & 4 & GNI KIV \\
\hline
\end{tabular}




\begin{tabular}{|c|c|c|c|c|}
\hline 010812090933 & 40.18 & 43.84 & 3.8 & GNI KIV \\
\hline 010819084933 & 42.68 & 44.08 & 4 & GNI KIV \\
\hline 010821081153 & 45.18 & 43.54 & 4.2 & GNI KIV \\
\hline 010824110251 & 42.87 & 38.2 & 3.3 & GNI KIV \\
\hline 010824114656 & 42.85 & 38.45 & 3 & GNI KIV \\
\hline 010829063523 & 47.27 & 43 & 3.5 & GNI KIV \\
\hline 010829133802 & 46.92 & 42.69 & 3.5 & GNI KIV \\
\hline 010902045036 & 46.04 & 42.07 & 3.8 & GNI \\
\hline 010902053127 & 45.95 & 42.09 & 4.2 & GNI \\
\hline 010902220543 & 40.1 & 38.7 & 3.9 & GNI \\
\hline 010904072748 & 46.11 & 43.07 & 3.7 & GNI \\
\hline 010905045905 & 42.73 & 44.08 & 4 & GNI \\
\hline 010905224123 & 45.82 & 42.44 & 4.2 & GNI KIV \\
\hline 010906104952 & 46.14 & 42.03 & 3.9 & GNI KIV \\
\hline 010906215603 & 48.51 & 42.88 & 3.9 & GNI KIV \\
\hline 010911020209 & 47.85 & 40.98 & 4.3 & GNI KIV \\
\hline 010911023727 & 46.58 & 42.04 & 4.1 & GNI KIV \\
\hline 010912152129 & 46.68 & 43.13 & 4 & GNI KIV \\
\hline 010912170820 & 42.52 & 39.11 & 3.2 & GNI KIV \\
\hline 010913122719 & 42.53 & 38.28 & 3.6 & GNI KIV \\
\hline 010913132949 & 42.58 & 38.34 & 4 & GNI KIV \\
\hline 010917050545 & 42.52 & 38.15 & 3.9 & GNI KIV \\
\hline 010917184623 & 43.64 & 42.76 & 4 & GNI KIV \\
\hline 010919235400 & 45.97 & 42 & 4.2 & GNI KIV \\
\hline 010923011408 & 49.22 & 39.92 & 4.3 & GNI KIV \\
\hline 010925154218 & 40.69 & 38.46 & 3.6 & GNI KIV \\
\hline 010925161919 & 46.07 & 43.08 & 3.8 & GNI KIV \\
\hline 010925173631 & 46.32 & 41.7 & 4.1 & GNI KIV \\
\hline 010928083022 & 46.26 & 42.3 & 4.3 & GNI KIV \\
\hline 011001124814 & 46.06 & 43.66 & 4 & GNI KIV \\
\hline 011001175217 & 47.33 & 41.62 & 4.3 & GNI KIV \\
\hline 011002010627 & 42.3 & 38.98 & 3.5 & GNI KIV \\
\hline 011004020800 & 42.69 & 40.19 & 3.4 & GNI KIV \\
\hline 011007220526 & 40.58 & 38.55 & 3.4 & GNI KIV \\
\hline 011010212231 & 40.13 & 39.09 & 3.6 & GNI KIV \\
\hline 011011204037 & 43.13 & 38.67 & 2.9 & GNI KIV \\
\hline 011012015149 & 40.1 & 39.19 & 3.5 & GNI KIV \\
\hline 011013080710 & 43.39 & 38.61 & 2.8 & GNI KIV \\
\hline 011014191306 & 45.95 & 42.1 & 4 & GNI KIV \\
\hline 011016110213 & 42.61 & 38.19 & 3.1 & GNI \\
\hline 011016231917 & 40.45 & 39.38 & 3.2 & GNI \\
\hline 011017025146 & 40.23 & 39.42 & 3.3 & GNI \\
\hline 011018215432 & 42.32 & 39.7 & 2.6 & GNI KIV \\
\hline 011022100923 & 46.6 & 42.92 & 4 & GNI KIV \\
\hline 011022121110 & 42.18 & 44.17 & 4 & GNI KIV \\
\hline 011024185109 & 40.58 & 38.79 & 2.7 & GNI KIV \\
\hline 011026133645 & 46.45 & 42.37 & 4.2 & GNI KIV \\
\hline 011026144653 & 42.05 & 42.46 & 4 & GNI KIV \\
\hline 011027003611 & 40.32 & 38.94 & 2.3 & GNI KIV \\
\hline 011028041105 & 45.85 & 42.76 & 4 & GNI KIV \\
\hline 011030043145 & 47.31 & 41.47 & 4.6 & GNI KIV \\
\hline 011030114327 & 41.23 & 39.19 & 3.1 & GNI KIV \\
\hline
\end{tabular}




\begin{tabular}{|c|c|c|c|c|}
\hline 011030224705 & 43.9 & 42.8 & 4 & GNI KIV \\
\hline 011101233653 & 48.67 & 42.64 & 4.3 & GNI KIV \\
\hline 011102000249 & 48.74 & 39.81 & 3.3 & GNI KIV \\
\hline 011102011128 & 43.89 & 42.72 & 4 & GNI KIV \\
\hline 011105160713 & 45.35 & 43.04 & 3.8 & GNI KIV \\
\hline 011108024937 & 46.6 & 41.64 & 3.9 & GNI KIV \\
\hline 011108073006 & 43.02 & 38.5 & 3.4 & GNI KIV \\
\hline 011110034839 & 41.67 & 38.4 & 3.2 & GNI KIV \\
\hline 011116024424 & 43.24 & 38.35 & 3.8 & GNI KIV \\
\hline 011123200126 & 45.46 & 42.23 & 4.1 & GNI KIV \\
\hline 011124033640 & 50.22 & 40.16 & 3.4 & GNI KIV \\
\hline 011126052423 & 48.71 & 40.63 & 4 & GNI KIV \\
\hline 011126070232 & 46.37 & 42.84 & 3.7 & GNI KIV \\
\hline 011128103542 & 41.63 & 39.66 & 3.8 & GNI KIV \\
\hline 011129193639 & 41.33 & 39.85 & 4.1 & NAR \\
\hline 011129193642 & 41.56 & 39.76 & 4.2 & GNI KIV \\
\hline 011201154116 & 41.66 & 39.48 & 3.8 & GNI KIV \\
\hline 011201220330 & 43.3 & 38.62 & 2.8 & GNI KIV \\
\hline 011202024646 & 43.57 & 38.65 & 2.9 & GNI KIV \\
\hline 011202041146 & 43.08 & 38.8 & 4.5 & AMB \\
\hline 011204094028 & 43.26 & 38.59 & 4.2 & GNI KIV \\
\hline 011204094031 & 43.13 & 38.75 & 3.7 & AMB NAR \\
\hline 011204094312 & 43.33 & 38.66 & 3.8 & GNI KIV \\
\hline 011208145709 & 44.81 & 43.34 & 4.3 & GNI KIV \\
\hline 011212010258 & 44.94 & 43.24 & 4.4 & GNI KIV \\
\hline 011215022636 & 50.01 & 40.19 & 3.8 & GNI KIV \\
\hline 011215043149 & 42.37 & 38.6 & 4 & GNI KIV \\
\hline 011215043151 & 41.9 & 39.37 & 4.2 & AMB \\
\hline 011215055730 & 42.48 & 38.84 & 3.1 & GNI KIV \\
\hline 011216193938 & 42.08 & 39.25 & 3.4 & GNI KIV \\
\hline 011220154930 & 40.09 & 39.27 & 3.4 & GNI KIV \\
\hline 011223072907 & 44.49 & 43.76 & 4.3 & GNI KIV \\
\hline 011223124143 & 44.46 & 43.9 & 3.8 & GNI KIV \\
\hline 011223124625 & 44.64 & 44.07 & 3.8 & GNI KIV \\
\hline 011223125009 & 44.54 & 43.96 & 3.9 & GNI KIV \\
\hline 011223133303 & 44.57 & 43.76 & 3.8 & GNI KIV \\
\hline 011223134518 & 44.71 & 43.86 & 3.9 & GNI KIV \\
\hline 011223151239 & 44.76 & 43.86 & 2.9 & GNI KIV \\
\hline 011223151633 & 44.71 & 43.85 & 4.1 & GNI KIV \\
\hline 011223221856 & 45.62 & 42.99 & 3.8 & GNI KIV \\
\hline 011224204110 & 48.34 & 39.86 & 3.7 & GNI KIV \\
\hline 011225060300 & 44.75 & 44 & 3.9 & GNI KIV \\
\hline 020102010813 & 47.5 & 41.92 & 3.8 & GNI KIV \\
\hline 020104214843 & 47.98 & 42.13 & 3.9 & GNI KIV \\
\hline 020105030816 & 50.4 & 41.02 & 3.6 & GNI KIV \\
\hline 020106153907 & 46.07 & 42.28 & 4.1 & GNI KIV \\
\hline 020107175318 & 43.79 & 40.78 & 3.5 & GNI KIV \\
\hline 020109013139 & 49.96 & 40.22 & 3.5 & GNI KIV \\
\hline 020110053147 & 45 & 43.59 & 3.5 & GNI KIV \\
\hline 020110141218 & 48.55 & 42.65 & 3.5 & GNI KIV \\
\hline 020112055249 & 45.52 & 44.22 & 3.8 & GNI KIV \\
\hline 020112075702 & 44.7 & 43.93 & 4 & GNI KIV \\
\hline
\end{tabular}




\begin{tabular}{|c|c|c|c|c|}
\hline 020113012509 & 46.58 & 42.09 & 3.9 & GNI KIV \\
\hline 020114122557 & 44.8 & 44.39 & 4 & GNI KIV \\
\hline 020116033555 & 41.88 & 39.04 & 3.1 & GNI KIV \\
\hline 020116201031 & 40.29 & 38.65 & 3.6 & GNI KIV \\
\hline 020117060825 & 40.31 & 38.68 & 3.5 & GNI KIV \\
\hline 020122005808 & 46.54 & 43.34 & 4 & GNI KIV \\
\hline 020123165345 & 40.49 & 38.6 & 3.2 & GNI KIV \\
\hline 020124035555 & 46.6 & 41.86 & 4.3 & GNI KIV \\
\hline 020124042726 & 43.05 & 43.48 & 4.1 & GNI KIV \\
\hline 020124072359 & 48.46 & 38.76 & 4.1 & GNI KIV \\
\hline 020124072738 & 47.94 & 38.09 & 3 & GNI KIV \\
\hline 020127001319 & 46.1 & 41.23 & 4 & GNI KIV \\
\hline 020127005022 & 44.24 & 43.75 & 4 & GNI KIV \\
\hline 020130064327 & 45.4 & 43.47 & 3.8 & GNI KIV \\
\hline 020130160802 & 50.24 & 40.16 & 3.6 & GNI KIV \\
\hline 020201064210 & 48.25 & 40.98 & 3.5 & GNI KIV \\
\hline 020202162458 & 48.1 & 41.43 & 4 & GNI KIV \\
\hline 020204015330 & 44.1 & 41.11 & 4.1 & GNI KIV \\
\hline 020204015335 & 44.18 & 41.08 & 3.8 & NPP \\
\hline 020204123633 & 46.77 & 43.23 & 4.3 & GNI KIV \\
\hline 020206073029 & 42.22 & 43.76 & 3.9 & GNI KIV \\
\hline 020209005234 & 40.3 & 39.16 & 3.1 & GNI KIV \\
\hline 020209033119 & 40.48 & 39.15 & 3.6 & GNI KIV \\
\hline 020209033418 & 40.3 & 39.18 & 3.5 & GNI KIV \\
\hline 020210005828 & 46.09 & 42.45 & 4.2 & GNI KIV \\
\hline 020210115632 & 45.33 & 43.15 & 4 & GNI KIV \\
\hline 020211161834 & 50.18 & 40.03 & 4.9 & GNI KIV \\
\hline 020211211956 & 50.18 & 39.99 & 4.1 & GNI KIV \\
\hline 020212013230 & 47.74 & 41.87 & 3.6 & GNI KIV \\
\hline 020212201202 & 45.27 & 42.23 & 4 & GNI KIV \\
\hline 020216131804 & 45.24 & 43.01 & 4 & GNI KIV \\
\hline 020219113946 & 50.2 & 40.92 & 3.5 & GNI KIV \\
\hline 020220023146 & 40.37 & 38.88 & 2.5 & GNI KIV \\
\hline 020220193358 & 48.34 & 42.67 & 3.8 & GNI KIV \\
\hline 020222020322 & 49.92 & 41.04 & 4.5 & GNI KIV \\
\hline 020222195212 & 49 & 41.38 & 4.4 & GNI KIV \\
\hline 020225002641 & 40.89 & 40.04 & 3.3 & KIV \\
\hline 020225123713 & 48.18 & 41.94 & 4 & GNI KIV \\
\hline 020226221810 & 48.17 & 41.86 & 4.2 & GNI KIV \\
\hline 020228211649 & 45.01 & 43.38 & 4.2 & GNI KIV \\
\hline 020302114714 & 46.56 & 39.92 & 4.3 & GNI KIV \\
\hline 020302145639 & 40.16 & 39.44 & 3.2 & GNI KIV \\
\hline 020305065425 & 45.38 & 38.37 & 3.8 & $\mathrm{AMB}$ \\
\hline 020305065426 & 45.24 & 38.33 & 4 & GNI KIV \\
\hline 020306191301 & 44.36 & 38.52 & 4.1 & GNI KIV \\
\hline 020306191359 & 44.58 & 38.33 & 4.3 & KAM \\
\hline 020307013114 & 44.08 & 38.3 & 3.9 & AMB \\
\hline 020314083042 & 44.61 & 39.34 & 3.8 & GNI KIV \\
\hline 020314125659 & 44.18 & 39.34 & 3.9 & GNI KIV \\
\hline 020317041546 & 45.78 & 41.83 & 4.1 & AMB GNI KIV \\
\hline 020318025548 & 42.08 & 39.25 & 3 & GNI KIV \\
\hline 020319010501 & 40.49 & 39.09 & 3 & GNI KIV \\
\hline
\end{tabular}




\begin{tabular}{|c|c|c|c|c|}
\hline 020319104508 & 42.16 & 43.9 & 3.7 & GNI KIV \\
\hline 020319120415 & 42.08 & 44.18 & 3.7 & GNI KIV \\
\hline 020319190129 & 40.46 & 38.98 & 3 & GNI KIV \\
\hline 020320103353 & 46.37 & 43.06 & 4.2 & GNI KIV \\
\hline 020321013643 & 44.69 & 43.86 & 4 & GNI KIV \\
\hline 020321101339 & 43.81 & 42.77 & 4.2 & GNI KIV \\
\hline 020323171707 & 45.77 & 41.96 & 3.8 & GNI KIV \\
\hline 020323174859 & 43.02 & 43.45 & 4.2 & GNI KIV \\
\hline 020323184252 & 43.64 & 40.17 & 3.5 & GNI KIV \\
\hline 020327023524 & 49.28 & 42.41 & 4.3 & GNI KIV \\
\hline 020327224026 & 40.02 & 38.21 & 2.8 & GNI KIV \\
\hline 020327235723 & 43.54 & 42.35 & 4 & GNI KIV \\
\hline 020329140319 & 46.03 & 41.28 & 3.6 & GNI KIV \\
\hline 020329230100 & 48.18 & 41.68 & 3.8 & GNI KIV \\
\hline 020330140207 & 41.63 & 44.24 & 3.8 & GNI KIV \\
\hline 020330181448 & 41.56 & 38.92 & 3.3 & GNI KIV \\
\hline 020331004917 & 43.38 & 42.32 & 3.8 & GNI KIV \\
\hline 020401012808 & 40.71 & 38.64 & 2.4 & GNI KIV \\
\hline 020404090754 & 45.79 & 43.22 & 4.2 & GNI KIV \\
\hline 020404141314 & 40.55 & 38.53 & 2.8 & GNI KIV \\
\hline 020405052604 & 43.05 & 43.45 & 4.1 & GNI KIV \\
\hline 020407014221 & 43.57 & 44.07 & 4.1 & GNI KIV \\
\hline 020407225030 & 45.25 & 38.4 & 4.6 & GNI KIV \\
\hline 020411004117 & 42.44 & 40.3 & 3.9 & GNI KIV \\
\hline 020411163824 & 44.71 & 41.57 & 4.3 & GNI KIV \\
\hline 020412155936 & 43.07 & 43.45 & 4 & GNI KIV \\
\hline 020414222340 & 50.04 & 40.31 & 3.7 & GNI KIV \\
\hline 020417214842 & 48.49 & 42.54 & 3.5 & GNI KIV \\
\hline 020418121447 & 46.57 & 40.02 & 3.6 & AMB \\
\hline 020418171812 & 40.32 & 39.09 & 2.9 & GNI KIV \\
\hline 020418183428 & 42.66 & 38.16 & 3.2 & GNI KIV \\
\hline 020418190147 & 42.66 & 38.13 & 3.1 & GNI \\
\hline 020419092219 & 48.01 & 42.69 & 4 & GNI KIV \\
\hline 020419170606 & 47.58 & 42.54 & 4 & GNI KIV \\
\hline 020421071744 & 47.21 & 43.03 & 3.5 & GNI KIV \\
\hline 020421174425 & 40.74 & 39.34 & 2.6 & GNI KIV \\
\hline 020421185608 & 42.81 & 38.54 & 2.9 & GNI KIV \\
\hline 020422130030 & 47.22 & 43.17 & 3.7 & GNI KIV \\
\hline 020423115515 & 45.61 & 43.61 & 4.3 & GNI KIV \\
\hline 020424051014 & 47.22 & 43.05 & 4.1 & GNI KIV \\
\hline 020425174122 & 44.82 & 41.76 & 4.3 & GNI KIV \\
\hline 020427184619 & 49.25 & 41.92 & 3.9 & GNI KIV \\
\hline 020428083221 & 46.35 & 40.84 & 4 & GNI KIV \\
\hline 020501180542 & 44.92 & 42.79 & 4.1 & GNI KIV \\
\hline 020505163140 & 40.24 & 39.7 & 3.3 & GNI KIV \\
\hline 020508045159 & 41.39 & 39.2 & 2.9 & GNI KIV \\
\hline 020508172402 & 40.39 & 38.5 & 2.9 & GNI KIV \\
\hline 020511003321 & 44.72 & 43.9 & 3.8 & GNI KIV \\
\hline 020513060738 & 48.17 & 42.93 & 3.9 & GNI KIV \\
\hline 020513165928 & 48.28 & 42.92 & 4.1 & GNI KIV \\
\hline 020515181744 & 46.02 & 43.34 & 3.8 & KIV \\
\hline 020516145233 & 44.26 & 43.15 & 3.8 & GNI KIV \\
\hline
\end{tabular}




\begin{tabular}{|c|c|c|c|c|}
\hline 020517121723 & 42.46 & 38.13 & 3.1 & GNI KIV \\
\hline 020518020935 & 42.77 & 44.03 & 4.1 & KIV \\
\hline 020518121747 & 40.19 & 38.85 & 2.9 & KIV \\
\hline 020525014504 & 43.73 & 39.04 & 3.1 & GNI KIV \\
\hline 020526190410 & 42.78 & 40.41 & 3.2 & GNI KIV \\
\hline 020529054948 & 48.06 & 39.29 & 3.4 & GNI KIV \\
\hline 020529234541 & 47.02 & 42.7 & 3.9 & GNI KIV \\
\hline 020531213813 & 48.76 & 40.44 & 4.2 & GNI KIV \\
\hline 020601054348 & 48.63 & 41.17 & 4 & GNI KIV \\
\hline 020602032658 & 49.87 & 40.17 & 4.6 & GNI KIV \\
\hline 020602122214 & 44.9 & 43.27 & 3.9 & GNI KIV \\
\hline 020602123923 & 44.65 & 42.84 & 4.3 & GNI KIV \\
\hline 020603185052 & 43.21 & 38.22 & 3.5 & GNI KIV \\
\hline 020605105443 & 49.1 & 40.26 & 4.1 & GNI KIV \\
\hline 020605111942 & 49.1 & 40.22 & 5 & GNI KIV \\
\hline 020605140742 & 49.11 & 40.31 & 4.8 & GNI KIV \\
\hline 020606001555 & 43.69 & 39.39 & 3.4 & GNI KIV \\
\hline 020606202931 & 40.18 & 38.81 & 2.6 & GNI KIV \\
\hline 020609031218 & 45.37 & 38.31 & 3.8 & GNI KIV \\
\hline 020610040517 & 43.01 & 38.78 & 3.4 & GNI KIV \\
\hline 020612133153 & 46.96 & 42.84 & 3.9 & GNI KIV \\
\hline 020612231115 & 41.94 & 39.89 & 3 & GNI KIV \\
\hline 020612235040 & 45.07 & 43.36 & 4.2 & GNI KIV \\
\hline 020613140555 & 42.59 & 38.96 & 2.8 & GNI KIV \\
\hline 020614145102 & 44.94 & 43.23 & 4.2 & GNI KIV \\
\hline 020615115128 & 47.83 & 42.99 & 3.7 & GNI KIV \\
\hline 020615222853 & 46.99 & 43.24 & 4 & GNI KIV \\
\hline 020615223433 & 46.93 & 43.2 & 3.5 & GNI KIV \\
\hline 020615231504 & 48.34 & 42.75 & 3.8 & GNI KIV \\
\hline 020616104142 & 46.39 & 42.51 & 4.5 & GNI KIV \\
\hline 020616213547 & 47.93 & 42.87 & 4.2 & GNI KIV \\
\hline 020619160845 & 46.86 & 42.82 & 3.7 & GNI KIV \\
\hline 020619161811 & 41.42 & 39.43 & 3.2 & GNI KIV \\
\hline 020620070733 & 48.55 & 41.75 & 3.9 & GNI KIV \\
\hline 020620135502 & 45 & 38.64 & 4.1 & GNI KIV \\
\hline 020620141702 & 46.2 & 38.55 & 4 & AMB \\
\hline 020620164858 & 47.47 & 42.69 & 3.7 & GNI KIV \\
\hline 020620201106 & 48.4 & 42.66 & 3.8 & GNI KIV \\
\hline 020621063451 & 43.73 & 43.4 & 3.9 & GNI KIV \\
\hline 020621084723 & 43.84 & 43.48 & 3.8 & GNI KIV \\
\hline 020621095208 & 43.63 & 43.38 & 3.9 & GNI KIV \\
\hline 020621135148 & 43.68 & 43.36 & 3.8 & GNI KIV \\
\hline 020623043803 & 44.63 & 43.82 & 3.8 & GNI KIV \\
\hline 020624201609 & 46.85 & 43.2 & 3.9 & GNI KIV \\
\hline 020627142909 & 46.84 & 43.29 & 4.2 & GNI KIV \\
\hline 020627144155 & 46.91 & 43.16 & 4.1 & GNI KIV \\
\hline 020627212449 & 41.17 & 38.98 & 3.9 & GNI KIV \\
\hline 020629050940 & 40.67 & 38.61 & 3.3 & GNI KIV \\
\hline 020629081032 & 47.72 & 42.87 & 3.7 & GNI KIV \\
\hline 020629101520 & 47.74 & 43.21 & 3.7 & GNI KIV \\
\hline 020629101532 & 47.75 & 43.41 & 4.1 & GNI KIV \\
\hline 020629101848 & 47.76 & 43.11 & 3.7 & GNI KIV \\
\hline
\end{tabular}




\begin{tabular}{|c|c|c|c|c|}
\hline 020629105600 & 47.95 & 43.22 & 4.3 & GNI KIV \\
\hline 020629111056 & 47.48 & 43.49 & 3.8 & GNI KIV \\
\hline 020701051758 & 42.3 & 39.9 & 4.1 & GNI KIV \\
\hline 020701052920 & 42.2 & 39.89 & 3.3 & GNI KIV \\
\hline 020701210344 & 46.48 & 41.87 & 3.9 & GNI \\
\hline 020701213324 & 42.25 & 39.88 & 3.5 & GNI \\
\hline 020702023150 & 40.48 & 38.63 & 2.7 & $\mathrm{GNI}$ \\
\hline 020702133335 & 48.48 & 42.51 & 3.8 & GNI \\
\hline 020702224726 & 49.41 & 42.08 & 3.9 & GNI \\
\hline 020704120249 & 41.3 & 39.86 & 3.1 & GNI KIV \\
\hline 020705203853 & 48.08 & 42.02 & 3.7 & GNI KIV \\
\hline 020706043039 & 42.36 & 41.13 & 4.1 & GNI KIV \\
\hline 020706043057 & 42.52 & 40.95 & 3.5 & AMB NAR \\
\hline 020707012504 & 40.23 & 39.35 & 3.1 & GNI KIV \\
\hline 020707151254 & 49.2 & 40.86 & 4.2 & GNI KIV \\
\hline 020710040517 & 43.01 & 38.78 & 3.4 & GNI KIV \\
\hline 020710172810 & 47.65 & 43.17 & 3.7 & GNI KIV \\
\hline 020711054301 & 47.11 & 43.01 & 3.8 & GNI KIV \\
\hline 020712231115 & 41.94 & 39.89 & 3 & GNI KIV \\
\hline 020713140555 & 42.59 & 38.96 & 2.8 & GNI KIV \\
\hline 020714005314 & 43.68 & 42.74 & 3.9 & GNI KIV \\
\hline 020714172026 & 40.49 & 39.09 & 2.9 & GNI KIV \\
\hline 020717132409 & 48.3 & 42.96 & 4.1 & GNI KIV \\
\hline 020717193038 & 46.68 & 42.79 & 3.8 & GNI KIV \\
\hline 020718001325 & 41.68 & 39.23 & 2.8 & GNI KIV \\
\hline 020718113431 & 46.98 & 42.88 & 3.8 & GNI KIV \\
\hline 020721004838 & 40.13 & 39.55 & 3.2 & GNI KIV \\
\hline 020721040618 & 43.18 & 39.43 & 3.6 & GNI KIV \\
\hline 020722192858 & 45.69 & 43.74 & 3.7 & KIV \\
\hline 020723124815 & 47.13 & 42.99 & 3.7 & GNI KIV \\
\hline 020725032049 & 44.36 & 42.84 & 3.8 & GNI KIV \\
\hline 020725125751 & 46.89 & 42.81 & 3.4 & GNI KIV \\
\hline 020725130122 & 42.03 & 44.05 & 3.5 & GNI KIV \\
\hline 020725212402 & 44.95 & 43.07 & 4 & GNI KIV \\
\hline 020726184552 & 41.34 & 43.12 & 4 & GNI KIV \\
\hline 020728015821 & 40.86 & 39.62 & 3 & GNI KIV \\
\hline 020728095548 & 42.68 & 43.15 & 3.5 & GNI KIV \\
\hline 020728142859 & 44.81 & 42.56 & 3.9 & GNI KIV \\
\hline 020729015024 & 44.96 & 42.85 & 3.8 & GNI KIV \\
\hline 020729020352 & 43.26 & 42.5 & 3.6 & GNI KIV \\
\hline 020730140527 & 41.03 & 39.2 & 3.9 & GNI KIV \\
\hline 020730222130 & 44.81 & 44.02 & 3.9 & GNI KIV \\
\hline 020731034207 & 44.05 & 44 & 3.5 & GNI KIV \\
\hline 020731042707 & 46.52 & 41.28 & 4 & GNI KIV \\
\hline 020731144058 & 50.54 & 40.01 & 3.9 & GNI KIV \\
\hline 020804051129 & 45.57 & 42.91 & 3.8 & GNI KIV \\
\hline 020807205700 & 45.27 & 42.11 & 3.9 & GNI KIV \\
\hline 020809022334 & 47.28 & 42.23 & 3.9 & GNI KIV \\
\hline 020809182858 & 43 & 38.71 & 3.4 & GNI KIV \\
\hline 020809224223 & 47.43 & 41.9 & 3.7 & GNI KIV \\
\hline 020810201037 & 48.87 & 41.35 & 4.5 & GNI KIV \\
\hline 020811065121 & 40.32 & 39.81 & 3.1 & GNI KIV \\
\hline
\end{tabular}




\begin{tabular}{|c|c|c|c|c|}
\hline 020816153015 & 46.19 & 41.9 & 4.3 & GNI KIV \\
\hline 020816175911 & 40.95 & 39.31 & 3.1 & GNI KIV \\
\hline 020817014501 & 42.52 & 39.2 & 3 & GNI KIV \\
\hline 020817132832 & 46.32 & 41.93 & 4.3 & GNI KIV \\
\hline 020817132902 & 46 & 41.83 & 4.2 & AMB \\
\hline 020817133731 & 46.09 & 41.96 & 3.7 & GNI KIV \\
\hline 020817135551 & 46.32 & 41.98 & 4.2 & GNI KIV \\
\hline 020817145538 & 46.27 & 41.84 & 4.7 & GNI KIV \\
\hline 020817174812 & 46.23 & 41.85 & 4.8 & GNI KIV \\
\hline 020817175811 & 46.22 & 41.88 & 4.1 & GNI KIV \\
\hline 020817175835 & 45.32 & 42.35 & 3.8 & AMB \\
\hline 020817180643 & 46.29 & 41.9 & 5 & GNI KIV \\
\hline 020817180709 & 44.67 & 42.43 & 3.8 & AMB \\
\hline 020817185738 & 46.4 & 41.78 & 4.1 & GNI KIV \\
\hline 020817190455 & 46.31 & 41.96 & 3.8 & GNI KIV \\
\hline 020817201051 & 45.68 & 42.15 & 3.7 & GNI KIV \\
\hline 020817210756 & 46.26 & 41.88 & 4.2 & GNI KIV \\
\hline 020818052220 & 40.6 & 39.4 & 3.2 & GNI KIV \\
\hline 020818092950 & 49.29 & 39.93 & 3.7 & GNI KIV \\
\hline 020818115228 & 42.47 & 40.8 & 4.6 & GNI KIV \\
\hline 020818115246 & 42.65 & 40.87 & 3.7 & AMB \\
\hline 020818182029 & 46.36 & 42 & 3.8 & GNI KIV \\
\hline 020819004826 & 40.2 & 39.91 & 2.4 & GNI KIV \\
\hline 020819051453 & 40.89 & 39.36 & 2.8 & GNI KIV \\
\hline 020819140500 & 46.6 & 42.87 & 3.7 & GNI KIV \\
\hline 020820012125 & 45.64 & 43.07 & 4.9 & GNI KIV \\
\hline 020820033722 & 40.29 & 40.66 & 3.1 & GNI KIV \\
\hline 020820131225 & 45.94 & 41.83 & 3.8 & GNI KIV \\
\hline 020820235130 & 46.12 & 41.82 & 4.2 & GNI KIV \\
\hline 020821060317 & 46.14 & 41.93 & 4 & GNI KIV \\
\hline 020822082502 & 43.73 & 43.09 & 3.9 & GNI KIV \\
\hline 020823191528 & 46.15 & 42.03 & 3.7 & GNI KIV \\
\hline 020823192508 & 46.1 & 41.94 & 3.8 & GNI KIV \\
\hline 020824054042 & 46.13 & 41.86 & 4 & GNI KIV \\
\hline 020824152630 & 40.24 & 39.46 & 3.7 & GNI KIV \\
\hline 020825020015 & 40.15 & 39.38 & 2.6 & GNI KIV \\
\hline 020825151726 & 47.45 & 41.88 & 3.9 & GNI KIV \\
\hline 020826063010 & 40.24 & 39.24 & 3.3 & GNI KIV \\
\hline 020826105403 & 40.72 & 39.37 & 2.9 & GNI KIV \\
\hline 020827104831 & 42.07 & 44.08 & 3.5 & GNI KIV \\
\hline 020828223624 & 47.39 & 41.75 & 4.1 & GNI KIV \\
\hline 020829053215 & 43.91 & 39.38 & 3.3 & GNI KIV \\
\hline 020829065141 & 42.56 & 39.89 & 3.6 & GNI KIV \\
\hline 020829065630 & 42.48 & 38.62 & 3.5 & AMB \\
\hline 020829103633 & 42.07 & 44.04 & 3.4 & GNI KIV \\
\hline 020829131857 & 46.17 & 43.32 & 4.3 & GNI KIV \\
\hline 020829185631 & 48.6 & 41 & 4.2 & GNI KIV \\
\hline 020829223627 & 45.99 & 41.78 & 3.8 & GNI KIV \\
\hline 020830181348 & 46.9 & 43.18 & 3.8 & GNI KIV \\
\hline 020831023152 & 42.4 & 39.68 & 3.9 & GNI KIV \\
\hline 020831111929 & 48.63 & 41.64 & 4.2 & GNI KIV \\
\hline 020831213223 & 40.36 & 39.49 & 2.9 & GNI KIV \\
\hline
\end{tabular}




\begin{tabular}{|c|c|c|c|c|}
\hline 021001212445 & 46.16 & 43.51 & 3.9 & GNI KIV \\
\hline 021002220208 & 41.62 & 39.71 & 3.3 & GNI KIV \\
\hline 021006142511 & 41.97 & 39.52 & 3 & GNI KIV \\
\hline 021006142554 & 41.9 & 39.59 & 3.5 & GNI KIV \\
\hline 021006145138 & 41.83 & 39.54 & 2.9 & GNI KIV \\
\hline 021010185558 & 48.14 & 40.92 & 2.9 & KIV \\
\hline 021012074627 & 46.88 & 42.95 & 3.6 & KIV \\
\hline 021013103712 & 47.08 & 43.01 & 3.7 & KIV \\
\hline 021016141610 & 46.06 & 41.91 & 4 & KIV \\
\hline 021016215942 & 41.1 & 38.72 & 3 & KIV \\
\hline 021017005302 & 40.229 & 39.4 & 4.1 & BNG KIV \\
\hline 021017005449 & 40.59 & 39.45 & 4.2 & KIV \\
\hline 021017010133 & 40.32 & 39.5 & 3.8 & KIV \\
\hline 021017010428 & 40.28 & 39.49 & 3.2 & KIV \\
\hline 021017010836 & 40.3 & 39.47 & 3.5 & KIV \\
\hline 021017011228 & 40.23 & 39.42 & 2.9 & KIV \\
\hline 021017011459 & 40.12 & 39.38 & 3.3 & KIV \\
\hline 021017012035 & 40.19 & 39.36 & 3 & KIV \\
\hline 021017012235 & 40.23 & 39.41 & 2.9 & KIV \\
\hline 021017012435 & 40.53 & 39.83 & 2.8 & KIV \\
\hline 021017013306 & 40.96 & 39.7 & 2.8 & KIV \\
\hline 021017015000 & 40.51 & 39.47 & 3.3 & KIV \\
\hline 021017015222 & 40.14 & 39.4 & 3.2 & KIV \\
\hline 021017015827 & 40.29 & 39.46 & 3.7 & KIV \\
\hline 021017021041 & 40.24 & 39.45 & 3.4 & KIV \\
\hline 021017023455 & 40.35 & 39.53 & 3.6 & KIV \\
\hline 021017102957 & 40.08 & 39.35 & 3.3 & KIV \\
\hline 021017160213 & 40.15 & 39.42 & 3.2 & KIV \\
\hline 021018012059 & 40.48 & 39.66 & 3 & KIV \\
\hline 021018222841 & 44.96 & 42.85 & 3.9 & KIV \\
\hline 021020061353 & 46.2 & 41.61 & 4.2 & KIV \\
\hline 021021212547 & 40.67 & 38.57 & 3.2 & KIV \\
\hline 021021213400 & 40.57 & 38.6 & 3 & KIV \\
\hline 021022155212 & 40.157 & 39.338 & 4.8 & BNG \\
\hline 021022155213 & 40.22 & 39.35 & 3.6 & KIV \\
\hline 021022160756 & 40.18 & 39.41 & 3.2 & KIV \\
\hline 021022164654 & 40.08 & 39.32 & 3.1 & KIV \\
\hline 021022171139 & 40.13 & 39.34 & 3.1 & KIV \\
\hline 021022175357 & 40.43 & 38.59 & 3.3 & KIV \\
\hline 021022190406 & 40.16 & 39.35 & 3.2 & KIV \\
\hline 021022203700 & 40.11 & 39.3 & 2.9 & KIV \\
\hline 021022231126 & 40.29 & 39.41 & 3.2 & KIV \\
\hline 021023042813 & 40.2 & 39.41 & 3.1 & KIV \\
\hline 021025040647 & 40.06 & 39.67 & 3.1 & KIV \\
\hline 021025074653 & 46.8 & 42.75 & 3.7 & KIV \\
\hline 021025172728 & 48.91 & 42.29 & 3.8 & KIV \\
\hline 021027054921 & 40.11 & 39.35 & 3.2 & KIV \\
\hline 021027210456 & 40.64 & 39.72 & 3.3 & KIV \\
\hline 021028224601 & 40.95 & 39.14 & 3.4 & KIV \\
\hline 021030020742 & 48.7 & 42.72 & 3.9 & KIV \\
\hline 021030100032 & 40.35 & 39.56 & 3.4 & KIV \\
\hline 021030120846 & 46.36 & 42.22 & 3.8 & KIV \\
\hline
\end{tabular}




\begin{tabular}{|c|c|c|c|c|}
\hline 021102053037 & 42.62 & 38.85 & 3.5 & KIV \\
\hline 021105115733 & 42.05 & 44.07 & 3.7 & KIV \\
\hline 021106065404 & 47.02 & 43.15 & 4.2 & KIV \\
\hline 021108010033 & 40.52 & 39.62 & 3.3 & KIV \\
\hline 021109154808 & 44.37 & 43.45 & 4 & KIV \\
\hline 021110063536 & 45.52 & 42.26 & 4.3 & KIV \\
\hline 021110072200 & 40.35 & 39.47 & 3 & KIV \\
\hline 021112064208 & 41.31 & 38.1 & 3.2 & KIV \\
\hline 021112082029 & 47.15 & 43.1 & 3.7 & KIV \\
\hline 021112235315 & 40.6 & 38.6 & 2.5 & KIV \\
\hline 021113172014 & 42.84 & 39.88 & 3.5 & KIV \\
\hline 021117095239 & 50.18 & 41.54 & 3.7 & KIV \\
\hline 021117201001 & 46.66 & 43.19 & 3.8 & KIV \\
\hline 021121121226 & 47.24 & 43.19 & 3.5 & KIV \\
\hline 021124101432 & 47.03 & 43.22 & 4.1 & KIV \\
\hline 021124105621 & 47.08 & 43.2 & 4.3 & KIV \\
\hline 021124115329 & 47.09 & 43.21 & 3.9 & KIV \\
\hline 021124160804 & 42.1 & 40.23 & 3.3 & KIV \\
\hline 021124181320 & 42.06 & 40.22 & 3.5 & KIV \\
\hline 021125031605 & 44.89 & 38.29 & 3.6 & KIV \\
\hline 021126025212 & 46.91 & 43.14 & 3.7 & KIV \\
\hline 021126052625 & 41.79 & 38.42 & 2.9 & KIV \\
\hline 021127121102 & 40.15 & 39.75 & 3 & KIV \\
\hline 021127215853 & 40.17 & 39.69 & 3.2 & KIV \\
\hline 021201062636 & 46.76 & 41.68 & 4.1 & KIV \\
\hline 021201235601 & 49.96 & 40.91 & 3.7 & KIV \\
\hline 021203164409 & 40.25 & 39.34 & 2.8 & KIV \\
\hline 021204093831 & 45.79 & 43.57 & 3.8 & KIV \\
\hline 021204194611 & 40.6 & 39.39 & 3.2 & KIV \\
\hline 021204195439 & 40.74 & 39.36 & 3 & KIV \\
\hline 021206104504 & 41.65 & 38.99 & 3.2 & KIV \\
\hline 021207022800 & 43.47 & 41.77 & 4.3 & KIV \\
\hline 021208224326 & 41.93 & 39.69 & 3.1 & KIV \\
\hline 021209063525 & 44.56 & 43.61 & 4.1 & KIV \\
\hline 021210213221 & 46.48 & 40.4 & 4.2 & KIV \\
\hline 021210213224 & 46.92 & 40.97 & 3.7 & AMB \\
\hline 021211084217 & 43.94 & 38.77 & 3.3 & KIV \\
\hline 021211084238 & 45.47 & 38.67 & 3.5 & AMB \\
\hline 021215110002 & 42.1 & 44.08 & 3.8 & KIV \\
\hline 021215231427 & 40.52 & 39.35 & 3.1 & KIV \\
\hline 021216051436 & 46.04 & 43.11 & 4.3 & KIV \\
\hline 021218004234 & 41.82 & 38.93 & 3 & KIV \\
\hline 021218035121 & 41.04 & 38.88 & 2.7 & KIV \\
\hline 021218120548 & 41.83 & 44.05 & 3.5 & KIV \\
\hline 021218235354 & 46.44 & 42.88 & 3.7 & KIV \\
\hline 021223121503 & 46.62 & 41.61 & 3.9 & KIV \\
\hline 021223121504 & 46.07 & 41.97 & 4.1 & AMB \\
\hline 021223160546 & 46.42 & 41.8 & 3.9 & KIV \\
\hline 021223184505 & 42.23 & 40.07 & 3.1 & KIV \\
\hline 021223213637 & 48.04 & 40.67 & 3.5 & KIV \\
\hline 021225223651 & 40.6 & 38.56 & 2.8 & KIV \\
\hline 021227021902 & 50.19 & 40.02 & 3.3 & KIV \\
\hline
\end{tabular}




\begin{tabular}{|c|c|c|c|c|}
\hline 021229165339 & 41.82 & 39.9 & 3 & KIV \\
\hline 021230212328 & 40.64 & 38.65 & 3.1 & KIV \\
\hline 030120163853 & 49.91 & 40.44 & 4.6 & GNI KIV \\
\hline 030127052622 & 39.773 & 39.456 & 6.2 & BNG ELZ ELZ ELZ TER \\
\hline 030203174018 & 46.21 & 43.1 & 3.9 & KIV \\
\hline 030205000735 & 46.62 & 42.79 & 3.8 & KIV \\
\hline 030206004804 & 44.03 & 41.53 & 12345 & GNI KIV \\
\hline 030206013301 & 45.56 & 42.69 & 12345 & GNI KIV \\
\hline 030206033133 & 44.08 & 41.54 & 12345 & GNI KIV \\
\hline 030206104818 & 42.05 & 44.07 & 3.9 & GNI KIV \\
\hline 030207062431 & 45.82 & 43.13 & 12345 & GNI KIV \\
\hline 030208063800 & 42.1 & 40.11 & 3.4 & GNI KIV \\
\hline 030209093144 & 43.68 & 41.39 & 12345 & GNI KIV \\
\hline 030211094740 & 40.05 & 39.42 & 3.3 & GNI KIV \\
\hline 030212142950 & 44.45 & 41.36 & 12345 & GNI KIV \\
\hline 030213214132 & 41.94 & 38.25 & 3 & GNI KIV \\
\hline 030215034224 & 44.02 & 41.18 & 12345 & GNI KIV \\
\hline 030215092820 & 40.74 & 39.34 & 2.9 & GNI KIV \\
\hline 030215193411 & 41.32 & 43.5 & 3.9 & GNI KIV \\
\hline 030216002917 & 42 & 38.2 & 2.7 & GNI KIV \\
\hline 030216204932 & 40.46 & 39.01 & 2.6 & GNI KIV \\
\hline 030217025540 & 46.44 & 41.82 & 3.9 & GNI KIV \\
\hline 030217033019 & 42.04 & 38.23 & 2.8 & GNI KIV \\
\hline 030218161034 & 44.45 & 43.66 & 3.9 & GNI KIV \\
\hline 030219140146 & 42.56 & 44.19 & 3.7 & GNI KIV \\
\hline 030220092734 & 43.89 & 39.48 & 3.7 & GNI KIV \\
\hline 030220142302 & 45.01 & 38.35 & 4 & GNI KIV \\
\hline 030220231516 & 43.89 & 41.13 & 12345 & GNI KIV \\
\hline 030221182941 & 43.94 & 41.59 & 12345 & GNI KIV \\
\hline 030224074528 & 45.99 & 43.31 & 3.5 & GNI KIV \\
\hline 030224215155 & 43.89 & 41.25 & 12345 & GNI KIV \\
\hline 030225212135 & 43.93 & 41.09 & 12345 & GNI KIV \\
\hline 030227123252 & 44.37 & 41.48 & 12345 & GNI KIV \\
\hline 030227223643 & 44.14 & 38.43 & 12345 & GNI KIV \\
\hline 030228030431 & 45.99 & 43.21 & 3.8 & GNI KIV \\
\hline 030301121915 & 46.1 & 43.51 & 4.2 & GNI KIV \\
\hline 030301123622 & 43.99 & 41.33 & 12345 & GNI KIV \\
\hline 030301165228 & 40.87 & 39.4 & 3.3 & GNI KIV \\
\hline 030302003831 & 45.72 & 42.87 & 3.7 & GNI KIV \\
\hline 030302074842 & 40.2 & 38.88 & 2.8 & GNI KIV \\
\hline 030302221443 & 43.65 & 38.55 & 3.4 & GNI KIV \\
\hline 030303000330 & 43.4 & 42.4 & 12345 & KIV \\
\hline 030303025541 & 44.39 & 43.49 & 3.9 & KIV \\
\hline 030304223650 & 43.98 & 41.37 & 12345 & GNI KIV \\
\hline 030305014204 & 43.89 & 41.47 & 12345 & GNI KIV \\
\hline 030306082312 & 41.26 & 38.59 & 3 & GNI KIV \\
\hline 030306213823 & 44.29 & 38.1 & 4.4 & GNI KIV \\
\hline 030309083700 & 43.98 & 41.37 & 12345 & GNI KIV \\
\hline 030310011534 & 46.62 & 41.31 & 3.7 & GNI KIV \\
\hline 030310022851 & 40.54 & 38.51 & 3.5 & GNI KIV \\
\hline
\end{tabular}




\begin{tabular}{|c|c|c|c|c|}
\hline 030310103639 & 46.94 & 42.98 & 3.6 & GNI KIV \\
\hline 030311042111 & 44.02 & 41.51 & 12345 & GNI KIV \\
\hline 030312043232 & 42.55 & 40.15 & 3.6 & GNI KIV \\
\hline 030312134827 & 44.05 & 41.62 & 12345 & GNI KIV \\
\hline 030312140853 & 44.38 & 41.33 & 12345 & GNI KIV \\
\hline 030313120829 & 43.82 & 42.86 & 12345 & GNI KIV \\
\hline 030314044736 & 43.89 & 41.39 & 12345 & GNI KIV \\
\hline 030314050545 & 43.9 & 41.32 & 12345 & GNI KIV \\
\hline 030314113227 & 44.08 & 41.49 & 12345 & GNI KIV \\
\hline 030314155729 & 46.25 & 43.01 & 4.2 & GNI KIV \\
\hline 030314184531 & 43.88 & 41.53 & 12345 & GNI KIV \\
\hline 030317030246 & 41.65 & 38.35 & 2.9 & GNI KIV \\
\hline 030318105358 & 43.8 & 41.46 & 12345 & GNI KIV \\
\hline 030320140341 & 44.49 & 41.4 & 12345 & GNI KIV \\
\hline 030323014041 & 40.61 & 38.48 & 3 & GNI KIV \\
\hline 030323142124 & 47.29 & 42.16 & 3.9 & GNI KIV \\
\hline 030323150942 & 43.48 & 41.08 & 12345 & GNI KIV \\
\hline 030323220107 & 44.25 & 43.98 & 4 & GNI KIV \\
\hline 030323220445 & 44.22 & 43.99 & 3.5 & GNI KIV \\
\hline 030323221621 & 44.29 & 43.99 & 3.9 & GNI KIV \\
\hline 030324025413 & 46.75 & 42.39 & 3.8 & GNI KIV \\
\hline 030325044538 & 44.12 & 43.93 & 3.6 & GNI KIV \\
\hline 030325081129 & 44.29 & 43.93 & 3.9 & GNI KIV \\
\hline 030325083436 & 43.67 & 43.6 & 3.9 & GNI KIV \\
\hline 030325111455 & 44.43 & 44.04 & 3.6 & GNI KIV \\
\hline 030325111801 & 44.41 & 44 & 3.7 & GNI KIV \\
\hline 030325115829 & 44.56 & 44.06 & 4 & GNI KIV \\
\hline 030327072621 & 47.2 & 43.05 & 4.4 & GNI KIV \\
\hline 030327072628 & 46.53 & 42.89 & 12345 & GNI KIV \\
\hline 030331053619 & 44.7 & 42.97 & 12345 & GNI KIV \\
\hline 030406102630 & 49.29 & 42.01 & 4.2 & GNI KIV \\
\hline 030410063952 & 48.75 & 42.28 & 4.1 & GNI KIV \\
\hline 030425004400 & 43.17 & 38.61 & 4 & GNI KIV \\
\hline 030501002704 & -12345 & -12345 & 6.4 & BIN BNG ELZ ELZ ELZ ERC GNI KIV KMR MLZ MLZ MLZ TAT TERP TER \\
\hline 030501004159 & 40.57 & 39.12 & 4.2 & GNI KIV \\
\hline 030501063443 & 40.35 & 39.17 & 4 & GNI KIV \\
\hline 030501093550 & 40.84 & 38.67 & 4.1 & GNI KIV \\
\hline 030504020958 & 40.56 & 39.06 & 4.4 & KIV \\
\hline 030508014420 & 40.37 & 39.07 & 4.6 & AJK BNG4 BNG GNI KIV \|LK TCK \\
\hline 030508150225 & 48.71 & 41.52 & 4.3 & GNI KIV \\
\hline 030510154451 & 40.36 & 39.049 & 4.5 & AJK BNG4 BNG GNI KIV \|LK \\
\hline 030510184451 & 40.36 & 39.05 & 4.5 & TCK \\
\hline 030510234142 & 40.44 & 39.05 & 3.6 & GNI KIV \\
\hline 030511234401 & 40.43 & 39.22 & 4 & GNI KIV \\
\hline 030512041010 & 40.57 & 38.93 & 4.3 & GNI KIV \\
\hline 030515063601 & 40.57 & 39.1 & 3 & GNI KIV \\
\hline 030515080626 & 40.51 & 38.94 & 3.5 & GNI KIV \\
\hline 030516114605 & 40.59 & 38.96 & 4.4 & GNI KIV \\
\hline 030601060945 & 47.38 & 41.07 & 4.7 & GNI KIV \\
\hline 030605171405 & 45.12 & 38.59 & 4.3 & GNI KIV \\
\hline
\end{tabular}




\begin{tabular}{|c|c|c|c|c|}
\hline 030725235931 & 50.17 & 40.21 & 3.9 & GNI KIV \\
\hline 030801045827 & 48.13 & 42.62 & 4 & GNI KIV \\
\hline 030811201208 & 44.88 & 38.83 & 4.7 & GNI KIV \\
\hline 030819050016 & 50.56 & 41.5 & 4.1 & KIV \\
\hline 031004012352 & 48.7 & 41.38 & 3.9 & GNI KIV \\
\hline 031004091055 & 49.73 & 40.21 & 4.1 & GNI KIV \\
\hline 031020062651 & 44.57 & 38.65 & 4.8 & GNI KIV \\
\hline 031024204722 & 50.21 & 40.68 & 4.6 & GNI KIV \\
\hline 031026080905 & 46.28 & 43.15 & 4 & GNI KIV \\
\hline 031110084343 & 48.98 & 41.08 & 4.7 & GNI KIV \\
\hline 031225201250 & 50.38 & 41.57 & 4.1 & KIV \\
\hline 040101072640 & 40.08 & 39.43 & 2.9 & GNI KIV \\
\hline 040101111023 & 40.06 & 39.34 & 3.1 & GNI KIV \\
\hline 040104232049 & 40.18 & 39.43 & 3.2 & GNI KIV \\
\hline 040104232959 & 42.05 & 39.13 & 3.5 & GNI KIV \\
\hline 040124044047 & 44.92 & 38.07 & 4.5 & KIV \\
\hline 040129112942 & 48.57 & 40.9 & 4.1 & GNI KIV \\
\hline 040208125411 & 47.4 & 41.31 & 4.1 & GNI KIV \\
\hline 040215093457 & 41.01 & 40.22 & 3.4 & GNI KIV \\
\hline 040215165716 & 41.02 & 40.28 & 3.7 & GNI KIV \\
\hline 040226041357 & 38.24 & 38.01 & 5 & DSH \\
\hline 040226061357 & 38.248 & 38.042 & 5 & MLT \\
\hline 040227162720 & 40.3 & 39.18 & 3.3 & GNI KIV \\
\hline 040228035133 & 40.15 & 38.75 & 3.2 & GNI KIV \\
\hline 040229013514 & 40.38 & 39.08 & 2.9 & GNI KIV \\
\hline 040229064108 & 40.52 & 38.95 & 3.4 & GNI KIV \\
\hline 040303143839 & 40.4 & 39.02 & 4.6 & GNI KIV \\
\hline 040325193049 & 40.91 & 39.74 & 5.1 & ERC GNI KIV TER \\
\hline 040325220922 & 40.8 & 39.64 & 4.4 & GNI KIV \\
\hline 040327044351 & 40.62 & 39.61 & 4 & GNI KIV \\
\hline 040328035109 & 40.86 & 39.87 & 5.3 & TER \\
\hline 040328035110 & 40.87 & 39.85 & 5.3 & GNI KIV \\
\hline 040328041521 & 40.76 & 39.41 & 4.2 & GNI KIV \\
\hline 040328042627 & 40.76 & 39.45 & 3.9 & GNI KIV \\
\hline 040328045503 & 40.78 & 39.12 & 4 & GNI KIV \\
\hline 040328051259 & 40.65 & 39.27 & 3.7 & GNI KIV \\
\hline 040401083112 & 40.78 & 39.93 & 4 & GNI KIV \\
\hline 040401083519 & 40.78 & 39.93 & 4.7 & GNI KIV \\
\hline 040401083521 & 40.669 & 39.857 & 4.6 & TER \\
\hline 040403124735 & 40.86 & 39.31 & 3.9 & GNI KIV \\
\hline 040418022122 & 45.86 & 42.31 & 3.7 & GNI KIV \\
\hline 040429194933 & 45.92 & 42.09 & 3.7 & GNI KIV \\
\hline 040501194850 & 40.722 & 39.935 & 4 & TER \\
\hline 040501194855 & 40.9 & 39.93 & 4.2 & GNI KIV \\
\hline 040502090715 & 46.12 & 42.2 & 3.8 & GNI KIV \\
\hline 040502090732 & 45.99 & 42.23 & 4 & GNI KIV \\
\hline 040512034204 & 47.76 & 41.81 & 4 & GNI KIV \\
\hline 040520112506 & 40.71 & 39.93 & 3.8 & GNI KIV \\
\hline 040520204502 & 40.75 & 38.55 & 3.1 & GNI KIV \\
\hline 040521115105 & 40.76 & 40.14 & 3.1 & GNI KIV \\
\hline 040523024913 & 48.56 & 40.77 & 4.1 & GNI KIV \\
\hline 040523113523 & 40.36 & 39.22 & 3 & GNI KIV \\
\hline
\end{tabular}




\begin{tabular}{|c|c|c|c|c|}
\hline 040524212824 & 40.85 & 39.98 & 3.2 & GNI KIV \\
\hline 040526082854 & 43.81 & 38.95 & 3 & GNI KIV \\
\hline 040530223604 & 40.76 & 39.91 & 3.1 & GNI KIV \\
\hline 040601154815 & 46.17 & 43.21 & 3.7 & GNI KIV \\
\hline 040612133751 & 39.601 & 38.614 & 4.4 & ELZ ELZ ELZ \\
\hline 040811154826 & 39.146 & 38.368 & 5.3 & MLT \\
\hline 040926210316 & 43.23 & 38.66 & 4 & VAN \\
\hline 761124122216 & -12345 & -12345 & $\begin{array}{l}7 \\
-\end{array}$ & MAK \\
\hline 790411121426 & -12345 & -12345 & 12345 & MUR \\
\hline 831030041228 & -12345 & -12345 & 6.6 & ERZ ERZ ERZ HRS \\
\hline 850812025444 & -12345 & -12345 & 12345 & KIG \\
\hline 880420035000 & -12345 & -12345 & 5.5 & MUR \\
\hline 881207074124 & -12345 & -12345 & 6.7 & GUK \\
\hline 881207074545 & -12345 & -12345 & 12345 & GUK \\
\hline 881219172936 & -12345 & -12345 & 12345 & DZHN DZHN DZHN NAB STPV STRS \\
\hline 881230132849 & -12345 & -12345 & 12345 & LEN NAB STPV STRS \\
\hline 881231040710 & -12345 & -12345 & 12345 & DZHN DZHN DZHN LEN MET NAB STPV STRS \\
\hline 890104072941 & -12345 & -12345 & 12345 & MET NAB STPV STRS \\
\hline 890108130023 & -12345 & -12345 & 12345 & DZHN DZHN DZHN MET NAB \\
\hline 890108165334 & -12345 & -12345 & 12345 & NAB \\
\hline 890330163624 & -12345 & -12345 & 12345 & STRS \\
\hline 900107065026 & 49.39 & 41.1 & 4.9 & KIV \\
\hline 900305025942 & 40.56 & 38.2 & 4.4 & KIV \\
\hline 900420233005 & 40.07 & 40.12 & 4.8 & KIV \\
\hline 900517164159 & 44.81 & 42.06 & 3.9 & KIV \\
\hline 900527182758 & 44.24 & 40.92 & 5.3 & KIV \\
\hline 900621222556 & 45.67 & 42.27 & 4.8 & KIV \\
\hline 900626034500 & -12345 & -12345 & 0 & SPIK STPV \\
\hline 900714162700 & -12345 & -12345 & 0 & SPIK \\
\hline 900720173028 & 48.36 & 41.81 & 4.3 & KIV \\
\hline 900723205454 & 46.18 & 42.93 & 4 & KIV \\
\hline 900802171248 & 48.17 & 38.68 & 3.9 & KIV \\
\hline 900802211720 & 48.52 & 38.57 & 4.1 & KIV \\
\hline 900922024602 & 46.32 & 42.38 & 4.3 & KIV \\
\hline 900924063514 & 48.15 & 38.16 & 4.6 & KIV \\
\hline 901215103800 & -12345 & -12345 & 12345 & SPIK \\
\hline 901216154540 & 43.72 & 41.37 & 4.2 & KIV \\
\hline 901216154551 & -12345 & -12345 & 5.4 & SAKH SBGD SBKR SBVR SLEN SPIK STPV STRS SVNZ SVNZ SVNZ \\
\hline 901220130300 & -12345 & -12345 & 12345 & SBVR \\
\hline 901223212850 & 44.32 & 42.08 & 4.1 & KIV \\
\hline 901223212851 & -12345 & -12345 & 12345 & STPV \\
\hline 910101191857 & 48.46 & 39.8 & 4.8 & KIV \\
\hline 910111060400 & -12345 & -12345 & 12345 & SPIK \\
\hline 910327221754 & 45.43 & 40.43 & 4.6 & KIV \\
\hline 910413015601 & -12345 & -12345 & 12345 & SBVR \\
\hline 910424105436 & 41.13 & 39.65 & 4.7 & KIV \\
\hline 910427033159 & 43.68 & 40.01 & 4.2 & KIV \\
\hline 910429001248 & -12345 & -12345 & 6.8 & SAKH SBGD SBVR SLEN STPV STRS \\
\hline 910429091248 & 43.67 & 42.41 & 6 & KIV \\
\hline 910429093740 & 43.86 & 42.54 & 5.2 & KIV \\
\hline
\end{tabular}




\begin{tabular}{|c|c|c|c|c|}
\hline 910429095923 & 43.39 & 42.53 & 4.6 & KIV \\
\hline 910429100112 & 43.86 & 42.39 & 4.9 & KIV \\
\hline 910429101535 & 43.91 & 42.79 & 4.8 & KIV \\
\hline 910429101552 & 44.2 & 42.02 & 4.7 & KIV \\
\hline 910429101941 & 43.52 & 42.28 & 4.5 & KIV \\
\hline 910429103046 & 43.6 & 43.45 & 4.3 & KIV \\
\hline 910429105241 & 44.05 & 42.67 & 5 & KIV \\
\hline 910429110429 & 43.98 & 42.47 & 4.3 & KIV \\
\hline 910429111015 & 43.79 & 42.47 & 4.8 & KIV \\
\hline 910429115110 & 43.8 & 42.49 & 4.9 & KIV \\
\hline 910429115955 & 43.93 & 42.49 & 5 & KIV \\
\hline 910429131242 & 42.75 & 42.51 & 3.8 & KIV \\
\hline 910429132710 & 43.96 & 41.74 & 4.2 & KIV \\
\hline 910429134958 & 43.34 & 42.6 & 4.6 & KIV \\
\hline 910429144309 & 43.9 & 42.47 & 5.4 & KIV \\
\hline 910429152847 & 43.36 & 42.35 & 4.3 & KIV \\
\hline 910429164843 & 44.27 & 42.94 & 4.3 & KIV \\
\hline 910429164958 & 43.94 & 42.66 & 4.5 & KIV \\
\hline 910429165850 & 43.56 & 42.75 & 4 & KIV \\
\hline 910429171028 & 43.46 & 42.37 & 4.6 & KIV \\
\hline 910429172126 & 44.14 & 42.72 & 3.5 & KIV \\
\hline 910429175459 & 43.97 & 42.49 & 4.9 & KIV \\
\hline 910429182315 & 43.74 & 42.58 & 5.5 & KIV \\
\hline 910429183041 & 43.89 & 42.51 & 5.8 & KIV \\
\hline 910429190702 & 43.71 & 42.49 & 4.4 & KIV \\
\hline 910429191956 & 43.98 & 42.79 & 4.4 & KIV \\
\hline 910429194454 & 43.96 & 42.6 & 4.7 & KIV \\
\hline 910429201206 & 43.96 & 42.38 & 4.3 & KIV \\
\hline 910429201948 & 43.43 & 42.75 & 3.8 & KIV \\
\hline 910429202446 & 43.8 & 42.47 & 4.8 & KIV \\
\hline 910429203254 & 43.33 & 42.54 & 5.5 & KIV \\
\hline 910429212413 & 43.67 & 42.34 & 3.8 & KIV \\
\hline 910429222822 & 43.82 & 42.59 & 4.9 & KIV \\
\hline 910429233227 & 43.96 & 42.38 & 4.8 & KIV \\
\hline 910430022826 & 43.99 & 41.99 & 3.9 & KIV \\
\hline 910430082957 & 43.51 & 42.33 & 4.2 & KIV \\
\hline 910430083227 & 43.61 & 42 & 4.4 & KIV \\
\hline 910430103944 & 43.26 & 42.56 & 3.8 & KIV \\
\hline 910430111528 & 43.5 & 42.51 & 4.8 & KIV \\
\hline 910430124452 & 42.82 & 42.77 & 4.3 & KIV \\
\hline 910430151907 & 43.9 & 42.45 & 4.1 & KIV \\
\hline 910430160739 & 43.23 & 42.57 & 4.6 & KIV \\
\hline 910501011546 & 45.58 & 43.61 & 3.8 & KIV \\
\hline 910501030651 & 43.82 & 42.43 & 4.5 & KIV \\
\hline 910501033814 & 43.34 & 42.4 & 4 & KIV \\
\hline 910501051335 & 43.45 & 42.57 & 4.8 & KIV \\
\hline 910501140447 & 43.69 & 42.56 & 3.8 & KIV \\
\hline 910501143754 & 43.38 & 42.45 & 3.8 & KIV \\
\hline 910501231910 & 43.88 & 42.42 & 4.1 & KIV \\
\hline 910502012529 & 43.98 & 42.55 & 5.4 & KIV \\
\hline 910502020731 & 45.17 & 41.34 & 4.3 & KIV \\
\hline 910502021800 & 43.89 & 42.24 & 4.1 & KIV \\
\hline
\end{tabular}




\begin{tabular}{|c|c|c|c|c|}
\hline 910502034227 & 43.57 & 42.79 & 4 & KIV \\
\hline 910502043054 & 43.19 & 42.49 & 3.9 & KIV \\
\hline 910502090038 & 43.73 & 42.54 & 4.1 & KIV \\
\hline 910502094444 & 43.44 & 42.48 & 4.5 & KIV \\
\hline 910503060036 & -12345 & -12345 & 12345 & SAMB \\
\hline 910503060837 & 43.32 & 42.52 & 4.6 & KIV \\
\hline 910503061254 & 43.81 & 41.99 & 4.2 & KIV \\
\hline 910503061255 & -12345 & -12345 & 12345 & SAMB \\
\hline 910503110701 & -12345 & -12345 & 0 & SONI \\
\hline 910503191447 & -12345 & -12345 & 0 & SONI \\
\hline 910503201937 & 43.25 & 42.67 & 5.5 & KIV \\
\hline 910503201939 & -12345 & -12345 & 5.6 & SAMB SIRI SONB SONI \\
\hline 910503203745 & -12345 & -12345 & 0 & SONB SONI \\
\hline 910503205007 & -12345 & -12345 & 0 & SAMB SONB SONI \\
\hline 910503223336 & -12345 & -12345 & 0 & SIRI SONB SONI \\
\hline 910503230225 & -12345 & -12345 & 0 & SAMB SONI \\
\hline 910503231141 & -12345 & -12345 & 0 & SAMB SONB SONI \\
\hline 910503234100 & 43.28 & 42.62 & 5.2 & KIV \\
\hline 910503234101 & -12345 & -12345 & 12345 & SAMB SIRI SONB SONI \\
\hline 910503235849 & -12345 & -12345 & 0 & SAMB \\
\hline 910504014125 & -12345 & -12345 & 0 & SONI \\
\hline 910504045335 & 43.49 & 42.18 & 4.2 & KIV \\
\hline 910504045338 & -12345 & -12345 & 12345 & SIRI SONB SONI \\
\hline 910504045619 & -12345 & -12345 & 12345 & SONB SONI \\
\hline 910504055100 & -12345 & -12345 & 0 & SAMB SONI \\
\hline 910504064521 & -12345 & -12345 & 0 & SONI \\
\hline 910504105333 & -12345 & -12345 & 0 & SONB SONI \\
\hline 910504144936 & -12345 & -12345 & 0 & SONI \\
\hline 910504185439 & -12345 & -12345 & 0 & SIRI \\
\hline 910504214531 & -12345 & -12345 & 0 & SONI \\
\hline 910504220517 & -12345 & -12345 & 0 & SAMB SONI \\
\hline 910505004017 & -12345 & -12345 & 0 & SIRI \\
\hline 910505034000 & -12345 & -12345 & 12345 & SAMB \\
\hline 910505051447 & -12345 & -12345 & 0 & SACK SONI \\
\hline 910505070757 & -12345 & -12345 & 0 & SIRI \\
\hline 910505104526 & -12345 & -12345 & 0 & SAMB \\
\hline 910505114545 & -12345 & -12345 & 0 & SAMB SONI \\
\hline 910505154002 & -12345 & -12345 & 0 & SAMB \\
\hline 910505175220 & -12345 & -12345 & 0 & SIRI \\
\hline 910505214746 & -12345 & -12345 & 0 & SIRI SONB \\
\hline 910506013526 & -12345 & -12345 & 12345 & SACK SAMB SONB SONI \\
\hline 910506013527 & 43.23 & 42.59 & 4.4 & KIV \\
\hline 910506025424 & 43.51 & 42.33 & 4 & KIV \\
\hline 910506043312 & -12345 & -12345 & 0 & SIRI \\
\hline 910506075456 & -12345 & -12345 & 12345 & SIRI \\
\hline 910506205546 & -12345 & -12345 & 0 & SIRI \\
\hline 910507000123 & -12345 & -12345 & 12345 & SACK SAMB SONI \\
\hline 910507001642 & -12345 & -12345 & 0 & SONI \\
\hline 910507090122 & 43.1 & 42.55 & 4.7 & KIV \\
\hline 910507125046 & -12345 & -12345 & 0 & SIRI \\
\hline 910507130628 & -12345 & -12345 & 0 & SIRI \\
\hline
\end{tabular}




\begin{tabular}{|c|c|c|c|c|}
\hline 910507233534 & -12345 & -12345 & 0 & SIRI SONI \\
\hline 910508004426 & -12345 & -12345 & 12345 & SIRI \\
\hline 910508115634 & -12345 & -12345 & 0 & SZEB SZEB SZEB \\
\hline 910508130458 & -12345 & -12345 & 0 & SAMB \\
\hline 910508155546 & -12345 & -12345 & 0 & SIRI \\
\hline 910509002428 & -12345 & -12345 & 0 & SZEB SZEB SZEB \\
\hline 910509024500 & -12345 & -12345 & 0 & SIRI SONI \\
\hline 910509100016 & -12345 & -12345 & 0 & SIRI \\
\hline 910510012514 & 43.19 & 42.58 & 5 & KIV \\
\hline 910510012517 & -12345 & -12345 & 12345 & SACK SAMB SZEB SZEB SZEB \\
\hline 910510023542 & -12345 & -12345 & 0 & SIRI \\
\hline 910510033522 & -12345 & -12345 & 0 & SZEB SZEB SZEB \\
\hline 910510041756 & -12345 & -12345 & 0 & SIRI \\
\hline 910510043536 & -12345 & -12345 & $\begin{array}{l}0 \\
-\end{array}$ & SIRI \\
\hline 910510190246 & -12345 & -12345 & 12345 & SIRI SONB SZEB SZEB SZEB \\
\hline 910510201152 & -12345 & -12345 & 0 & SIRI \\
\hline 910510203043 & 43.41 & 42.51 & 4.4 & KIV \\
\hline 910510203044 & -12345 & -12345 & 12345 & SACK SAMB SIRI SONB SZEB SZEB SZEB \\
\hline 910510205226 & 43.96 & 42.45 & 5 & KIV \\
\hline 910510220021 & -12345 & -12345 & 0 & SZEB SZEB SZEB \\
\hline 910510234036 & -12345 & -12345 & 0 & SACK SIRI \\
\hline 910511055727 & -12345 & -12345 & 0 & SAMB SONB SZEB SZEB SZEB \\
\hline 910511143403 & -12345 & -12345 & 0 & SONB SONI \\
\hline 910511183514 & 43.88 & 42.38 & 3.8 & KIV \\
\hline 910511195418 & -12345 & -12345 & 0 & SIRI \\
\hline 910512044405 & -12345 & -12345 & 0 & SONB SONI \\
\hline 910512195600 & -12345 & -12345 & 0 & SIRI SONB SONI \\
\hline 910513003151 & -12345 & -12345 & 0 & SIRI \\
\hline 910514001029 & -12345 & -12345 & 0 & SIRI \\
\hline 910514003625 & -12345 & -12345 & 12345 & SIRI SONI SZEB SZEB SZEB \\
\hline 910514013002 & -12345 & -12345 & 0 & SIRI \\
\hline 910514065955 & -12345 & -12345 & 0 & SIRI \\
\hline 910514093625 & 43.46 & 42.47 & 4.4 & KIV \\
\hline 910514163610 & -12345 & -12345 & 0 & SONB SONI \\
\hline 910514191337 & -12345 & -12345 & 0 & SIRI \\
\hline 910514213330 & -12345 & -12345 & 0 & SIRI \\
\hline 910514214100 & -12345 & -12345 & 0 & SAMB SONI SZEB SZEB SZEB \\
\hline 910515003249 & -12345 & -12345 & 0 & SIRI SONI \\
\hline 910515142848 & 43.35 & 42.54 & 4.9 & KIV \\
\hline 910515142849 & -12345 & -12345 & 12345 & SAMB SIRI SONB SONI SZEB SZEB SZEB \\
\hline 910515145747 & -12345 & -12345 & 0 & SIRI \\
\hline 910516013000 & -12345 & -12345 & 0 & SONI SZEB SZEB SZEB \\
\hline 910516110444 & -12345 & -12345 & 0 & SONI \\
\hline 910516141828 & -12345 & -12345 & 0 & SIRI \\
\hline 910516200253 & -12345 & -12345 & 0 & SIRI \\
\hline 910517110544 & -12345 & -12345 & 0 & SIRI \\
\hline 910517141434 & 43.78 & 42.27 & 4.3 & KIV \\
\hline 910517142819 & -12345 & -12345 & 12345 & SAMB \\
\hline 910518051237 & -12345 & -12345 & 0 & SIRI \\
\hline 910518064839 & -12345 & -12345 & 0 & SZEB SZEB SZEB \\
\hline 910518203811 & -12345 & -12345 & 0 & SIRI \\
\hline
\end{tabular}




\begin{tabular}{|c|c|c|c|c|}
\hline 910521021725 & -12345 & -12345 & 0 & SIRI \\
\hline 910521064350 & -12345 & -12345 & 0 & SONB SONI SZEB SZEB SZEB \\
\hline 910521173743 & 47.88 & 42.74 & 5 & KIV \\
\hline 910521194813 & -12345 & -12345 & 0 & SIRI \\
\hline 910522013701 & -12345 & -12345 & 0 & SIRI SONB SONI SZEB SZEB SZEB \\
\hline 910523004134 & -12345 & -12345 & 12345 & SAMB SONB SONI SZEB SZEB SZEB \\
\hline 910523084131 & 43.23 & 42.51 & 4.4 & KIV \\
\hline 910523113853 & -12345 & -12345 & 0 & SZEB SZEB SZEB \\
\hline 910523152606 & -12345 & -12345 & 0 & SIRI \\
\hline 910523215131 & -12345 & -12345 & 0 & SZEB SZEB SZEB \\
\hline 910524075931 & -12345 & -12345 & 0 & SIRI SONI \\
\hline 910524075932 & 43.62 & 42.35 & 3.9 & KIV \\
\hline 910524141636 & -12345 & -12345 & 0 & SZEB SZEB SZEB \\
\hline 910525021352 & -12345 & -12345 & 0 & SIRI SONI \\
\hline 910526062050 & -12345 & -12345 & 0 & SZEB SZEB SZEB \\
\hline 910527034043 & 46.09 & 42.12 & 3.7 & KIV \\
\hline 910527052527 & -12345 & -12345 & 0 & SZEB SZEB SZEB \\
\hline 910527190030 & -12345 & -12345 & 0 & SZEB SZEB SZEB \\
\hline 910528044337 & -12345 & -12345 & 0 & SONI \\
\hline 910528195605 & -12345 & -12345 & 0 & SZEB SZEB SZEB \\
\hline 910530011030 & -12345 & -12345 & 0 & SZEB SZEB SZEB \\
\hline 910601021303 & -12345 & -12345 & 12345 & SZEB SZEB SZEB \\
\hline 910602061048 & -12345 & -12345 & 12345 & SONB SONI SZEB SZEB SZEB \\
\hline 910603000044 & -12345 & -12345 & 0 & SZEB SZEB SZEB \\
\hline 910603102241 & 42.85 & 40.07 & 4.5 & KIV \\
\hline 910604005516 & 42.99 & 40.62 & 3.7 & KIV \\
\hline 910605165515 & -12345 & -12345 & 0 & SIRI \\
\hline 910606002556 & -12345 & -12345 & 0 & SIRI \\
\hline 910606005645 & -12345 & -12345 & 0 & SIRI \\
\hline 910606132003 & -12345 & -12345 & 0 & SIRI SONI \\
\hline 910606134810 & -12345 & -12345 & 12345 & SIRI SONI \\
\hline 910606191007 & -12345 & -12345 & 0 & SIRI \\
\hline 910607004527 & -12345 & -12345 & 0 & SONI \\
\hline 910607145646 & -12345 & -12345 & 0 & SONI \\
\hline 910608011204 & 42.81 & 40.4 & 4.2 & KIV \\
\hline 910609030152 & -12345 & -12345 & 0 & SIRI SONB SONI \\
\hline 910609114630 & -12345 & -12345 & 0 & SIRI \\
\hline 910609200600 & -12345 & -12345 & 0 & SAMB \\
\hline 910610055755 & -12345 & -12345 & 0 & SIRI \\
\hline 910610133152 & -12345 & -12345 & 0 & SIRI \\
\hline 910611130638 & -12345 & -12345 & 0 & SIRI \\
\hline 910611225843 & -12345 & -12345 & 0 & SAMB \\
\hline 910615005920 & 44.02 & 42.44 & 6.1 & KIV SAMB SBVR SIRI SONB SONI SPIK STPV SZEB SZEB SZEB \\
\hline 910615202853 & -12345 & -12345 & 0 & SONI SZEB SZEB SZEB \\
\hline 910615214839 & -12345 & -12345 & 12345 & SIRI SONB SONI SZEB SZEB SZEB \\
\hline 910616045354 & -12345 & -12345 & 12345 & SPIK \\
\hline 910616110711 & 42.97 & 40.1 & 4.1 & KIV \\
\hline 910617025741 & -12345 & -12345 & 0 & SZEB SZEB SZEB \\
\hline 910617030445 & 44.1 & 42.26 & 4.4 & KIV \\
\hline 910617054234 & -12345 & -12345 & 0 & SONB SONI SZEB SZEB SZEB \\
\hline 910617185551 & -12345 & -12345 & 0 & SONI \\
\hline
\end{tabular}




\begin{tabular}{|c|c|c|c|c|}
\hline 910619064030 & 42.92 & 40.25 & 4.6 & KIV \\
\hline 910620001043 & -12345 & -12345 & 0 & SONI \\
\hline 910620001918 & -12345 & -12345 & 0 & SONI \\
\hline 910621181713 & -12345 & -12345 & 0 & SONB SONI \\
\hline 910621203912 & -12345 & -12345 & 0 & SIRI \\
\hline 910621221819 & -12345 & -12345 & 0 & SONB SONI \\
\hline 910625153632 & -12345 & -12345 & 0 & SZEB SZEB SZEB \\
\hline 910625182036 & -12345 & -12345 & 12345 & SIRI \\
\hline 910626025328 & -12345 & -12345 & 0 & SIRI \\
\hline 910629034007 & -12345 & -12345 & 0 & SIRI \\
\hline 910630200020 & -12345 & -12345 & 12345 & SIRI SONB SONI SZEB SZEB SZEB \\
\hline 910630200919 & 43.68 & 42.44 & 4.5 & KIV \\
\hline 910703130030 & -12345 & -12345 & 0 & SIRI \\
\hline 910704062629 & -12345 & -12345 & 5.4 & SIRI SONI SZEB SZEB SZEB \\
\hline 910704062630 & 44.14 & 42.38 & 5.3 & KIV \\
\hline 910706001223 & -12345 & -12345 & 0 & SZEB SZEB SZEB \\
\hline 910709061450 & -12345 & -12345 & 0 & SIRI SONB SONI \\
\hline 910711055818 & 43.65 & 41.94 & 4.6 & KIV \\
\hline 910711055820 & -12345 & -12345 & 12345 & SIRI SONB SONI SZEB SZEB SZEB \\
\hline 910711211215 & -12345 & -12345 & 0 & SONB SONI SZEB SZEB SZEB \\
\hline 910711211911 & -12345 & -12345 & 0 & SONI \\
\hline 910711212425 & -12345 & -12345 & 0 & SONB SONI SZEB SZEB SZEB \\
\hline 910711213212 & -12345 & -12345 & 0 & SONI \\
\hline 910715010258 & -12345 & -12345 & 0 & SONI \\
\hline 910716102549 & -12345 & -12345 & 0 & SZEB SZEB SZEB \\
\hline 910718071328 & -12345 & -12345 & 0 & SONI SZEB SZEB SZEB \\
\hline 910718102751 & -12345 & -12345 & 0 & SONI SZEB SZEB SZEB \\
\hline 910720181729 & -12345 & -12345 & 0 & SONB SONI SZEB SZEB SZEB \\
\hline 910722002121 & -12345 & -12345 & 0 & STRS \\
\hline 910724032401 & -12345 & -12345 & 0 & SPIK \\
\hline 910728192122 & -12345 & -12345 & 0 & SZEB SZEB SZEB \\
\hline 910730111841 & -12345 & -12345 & 0 & SZEB SZEB SZEB \\
\hline 910806111825 & -12345 & -12345 & 0 & SONB SONI \\
\hline 910806155311 & 43.88 & 42.39 & 4.3 & GNI KIV \\
\hline 910807145850 & -12345 & -12345 & 0 & SZEB SZEB SZEB \\
\hline 910809181514 & -12345 & -12345 & 0 & SONB SONI \\
\hline 910810085752 & 42.05 & 39.94 & 4.4 & KIV \\
\hline 910813074038 & -12345 & -12345 & 0 & SZEB SZEB SZEB \\
\hline 910815042239 & 40.95 & 39.08 & 3.7 & KIV \\
\hline 910815184000 & -12345 & -12345 & 0 & SONB SONI \\
\hline 910819142853 & -12345 & -12345 & 0 & SONI SZEB SZEB SZEB \\
\hline 910820165636 & -12345 & -12345 & 12345 & SONI \\
\hline 910905192310 & 41.58 & 39.03 & 4.3 & GNI KIV \\
\hline 910914054219 & -12345 & -12345 & 12345 & STRS \\
\hline 911006014645 & 43.45 & 41.15 & 4.9 & KIV \\
\hline 911006014647 & -12345 & -12345 & 5.1 & SAKH SBGD SBVR SLEN STRS \\
\hline 911010024446 & -12345 & -12345 & 12345 & SBGD \\
\hline 911010024450 & 43.44 & 41.38 & 4.4 & KIV \\
\hline 911018162340 & 47.75 & 41.71 & 4 & KIV \\
\hline 911021115822 & 46.77 & 41.82 & 4.7 & KIV \\
\hline 911112203604 & 45.06 & 38.97 & 4.3 & KIV \\
\hline
\end{tabular}




\begin{tabular}{|c|c|c|c|c|}
\hline 911208010206 & 48.12 & 38.32 & 3.8 & KIV \\
\hline 911220061526 & -12345 & -12345 & 12345 & SBGD SBVR \\
\hline 911227202504 & 43.84 & 42.4 & 3.7 & KIV \\
\hline 920127081132 & 44.08 & 42.31 & 4.5 & KIV KUB NAG \\
\hline 920127122150 & 42.19 & 40 & 3.7 & KIV KUB NAG \\
\hline 920214203157 & -12345 & -12345 & 0 & SPIK \\
\hline 920215125254 & 46.53 & 42.84 & 4.7 & GUM KIV MIC NAG \\
\hline 920215133034 & 46.6 & 42.66 & 4.2 & GUM KIV MIC NAG \\
\hline 920215133740 & 46.49 & 42.91 & 4.2 & GUM KIV MIC NAG \\
\hline 920215151804 & 46.21 & 41.49 & 4.4 & GUM KIV MIC NAG \\
\hline 920216143355 & 46.54 & 42.78 & 3.5 & GUM KIV MIC NAG \\
\hline 920218013940 & 43.57 & 41.22 & 4.5 & KIV MIC NAG \\
\hline 920225220156 & 45.27 & 39.22 & 3.2 & $\mathrm{KIV}$ \\
\hline 920311022608 & 46.56 & 42.77 & 4.4 & KIV MIC NAG \\
\hline 920313171840 & -12345 & -12345 & 6.6 & ERC REF TER \\
\hline 920315001200 & -12345 & -12345 & 12345 & SERA \\
\hline 920315014003 & -12345 & -12345 & 0 & STPV \\
\hline 920315152721 & -12345 & -12345 & 0 & SERA \\
\hline 920315161616 & -12345 & -12345 & 5.2 & ERC SERE \\
\hline 920315161625 & 39.93 & 39.53 & 5.4 & ERC \\
\hline 920315201234 & 40.25 & 39.81 & 3.8 & KIV \\
\hline 920319191639 & 45.78 & 43.1 & 4.5 & GUM KIV MIC NAG \\
\hline 920325035826 & -12345 & -12345 & 12345 & ERC1 \\
\hline 920325061156 & -12345 & -12345 & 0 & ERC1 \\
\hline 920325071111 & -12345 & -12345 & 0 & ERC1 \\
\hline 920325210632 & -12345 & -12345 & 0 & ERC1 \\
\hline 920326023246 & 43.6 & 42.3 & 3.6 & KIV MIC NAG \\
\hline 920327192101 & 43.79 & 42.46 & 5.1 & GUM KIV KUB MIC NAG \\
\hline 920327210256 & 43.69 & 42.3 & 3.6 & GUM KIV KUB MIC NAG \\
\hline 920329002620 & -12345 & -12345 & 12345 & ERC2 \\
\hline 920330235951 & -12345 & -12345 & 0 & ERC2 \\
\hline 920331000037 & -12345 & -12345 & 0 & ERC2 \\
\hline 920401173130 & -12345 & -12345 & 0 & ERC2 \\
\hline 920402122613 & -12345 & -12345 & 12345 & ERC2 \\
\hline 920404053405 & -12345 & -12345 & 0 & ERC2 \\
\hline 920404110652 & 42.89 & 39.61 & 3.9 & KIV \\
\hline 920412152224 & -12345 & -12345 & 0 & ERC2 \\
\hline 920418005723 & -12345 & -12345 & 0 & STPV \\
\hline 920419101440 & -12345 & -12345 & 12345 & $\mathrm{ERC} 2$ \\
\hline 920419232328 & -12345 & -12345 & $\begin{array}{l}0 \\
-\end{array}$ & ERC2 \\
\hline 920420180500 & -12345 & -12345 & 12345 & ERC2 \\
\hline 920422030352 & -12345 & -12345 & 12345 & ERC2 \\
\hline 920422053806 & -12345 & -12345 & 0 & ERC2 \\
\hline 920425003314 & -12345 & -12345 & 0 & ERC2 \\
\hline 920428071629 & -12345 & -12345 & 12345 & ERC2 \\
\hline 920507191505 & 40.09 & 38.66 & 5.1 & GUM KIV MIC NAG \\
\hline 920512165833 & 47.33 & 40.96 & 4 & GUM KIV MIC NAG \\
\hline 920514143304 & 40.47 & 40.81 & 3.6 & KIV \\
\hline 920714042630 & 41.74 & 39.29 & 4.5 & GNI GUM KIV \\
\hline 920714184623 & 41.78 & 39.23 & 4.5 & GUM KIV \\
\hline 920806175309 & 48.88 & 40.21 & 4.2 & KIV \\
\hline
\end{tabular}




\begin{tabular}{|c|c|c|c|c|}
\hline 920810021926 & 40.38 & 39.25 & 4.2 & GUM KIV KUB NAG \\
\hline 920821191107 & 43.26 & 42.48 & 4.8 & GUM KIV KUB NAG \\
\hline 920925155347 & 46.05 & 42.52 & 3.7 & GUM KIV KUB \\
\hline 921023231945 & 45.12 & 42.57 & 6 & KIV \\
\hline 921023231947 & -12345 & -12345 & 6.4 & SBGD STPV SVNZ SVNZ SVNZ \\
\hline 921024001437 & 45.07 & 42.63 & 4.2 & KIV \\
\hline 921024004653 & 44.89 & 42.43 & 3.5 & KIV \\
\hline 921105154227 & 45.09 & 42.45 & 4.5 & KIV \\
\hline 921106152846 & 47.78 & 38.04 & 4.1 & KIV \\
\hline 921208053426 & 45.42 & 40.26 & 4.2 & GUM KIV KUB NAG \\
\hline 921209202947 & 45.34 & 40.05 & 4.8 & GUM KIV MIC NAG \\
\hline 921211113737 & 43.52 & 42.42 & 4.2 & GUM KIV \\
\hline 921217224252 & -12345 & -12345 & 0 & STPV \\
\hline 921228175704 & 43.49 & 42.52 & 3.2 & GUM KIV NAG \\
\hline 930107101203 & 42.28 & 40.66 & 4 & GUM KIV \\
\hline 930117094750 & 41.69 & 40.76 & 4.3 & GUM KIV NAG \\
\hline 930120060438 & -12345 & -12345 & 12345 & STPV \\
\hline 930120173417 & -12345 & -12345 & 0 & ERC \\
\hline 930210223809 & 43.54 & 42.25 & 3.7 & KIV NAG \\
\hline 930212192608 & 40.52 & 40.17 & 3.8 & KIV KUB NAG \\
\hline 930219181328 & 45.01 & 40.57 & 4.6 & KIV KUB NAG \\
\hline 930222042421 & 43.86 & 42.56 & 5.1 & KIV KUB NAG \\
\hline 930310160322 & 43.7 & 42.46 & 4 & KIV NAG \\
\hline 930315153238 & 45.83 & 38.13 & 5 & KIV \\
\hline 930317235915 & 44.86 & 38.25 & 4.4 & KIV \\
\hline 930319200521 & 46.76 & 41.92 & 4.8 & GUM KIV KUB MIC NAG \\
\hline 930329005819 & 45.36 & 42.67 & 4.9 & GUM KIV KUB MIC NAG \\
\hline 930330222523 & 45 & 38.31 & 4.8 & GUM KIV KUB NAG \\
\hline 930419051947 & 43.62 & 44.21 & 4.8 & GUM KIV KUB MIC NAG \\
\hline 930424122919 & 44.09 & 41.36 & 4.5 & GUM KIV KUB MIC NAG \\
\hline 930427200703 & 43.83 & 42.69 & 4.7 & GUM KIV KUB MIC \\
\hline 930501064747 & 48.89 & 38.95 & 4.1 & KIV \\
\hline 930504223923 & 45.17 & 42.57 & 4.9 & GUM KIV \\
\hline 930604215623 & 43.31 & 42.63 & 4.5 & GUM KIV MIC NAG \\
\hline 930606015116 & -12345 & -12345 & 12345 & ERC \\
\hline 930607071427 & 46.33 & 40 & 4.7 & GUM KIV NAG \\
\hline 930621003253 & 40.43 & 39.42 & 3.8 & GUM KIV MIC NAG \\
\hline 930712162402 & -12345 & -12345 & 0 & ERC \\
\hline 930722015457 & 40.16 & 39.52 & 4.3 & GUM KIV KUB NAG \\
\hline 930831065532 & 49.48 & 41.87 & 5.8 & GUM KIV KNG \\
\hline 930923075646 & 42.5 & 41.37 & 3.7 & GUM KIV KNG KUB MIC NAG \\
\hline 930924125634 & -12345 & -12345 & 0 & STPV \\
\hline 931015223719 & 48.18 & 40.83 & 4.7 & GUM KIV MIC NAG \\
\hline 931025143321 & 49.43 & 41.29 & 4.7 & KIV NAG \\
\hline 940114162318 & -12345 & -12345 & 0 & STPV \\
\hline 940211174010 & 43.71 & 42.4 & 4.7 & GUM KIV MIC NAG \\
\hline 940224223225 & 40.52 & 40.04 & 4.1 & GNI KIV \\
\hline 940304230813 & 45.27 & 41.75 & 4.4 & KIV \\
\hline 940322110308 & 45.76 & 42.45 & 4 & GNI KIV \\
\hline 940410134047 & 46.12 & 43.04 & 4.8 & GNI KIV \\
\hline 940417080232 & 46.29 & 41.92 & 5 & GNI KIV \\
\hline 940420222552 & 43.42 & 42.72 & 3.3 & GNI GUM KIV MIC NAG \\
\hline
\end{tabular}




\begin{tabular}{|c|c|c|c|c|}
\hline 940502181403 & 42.94 & 40.21 & 3.9 & GUM KIV MIC NAG \\
\hline 940518171854 & 43.98 & 41.26 & 4.5 & GNI GUM KIV NAG \\
\hline 940605012001 & 43.4 & 42.47 & 4.3 & GNI GUM KIV MIC NAG \\
\hline 940625050048 & 43.71 & 42.09 & 4.4 & GNI KIV \\
\hline 940630095511 & 50.55 & 40.93 & 4.9 & GNI KIV \\
\hline 940701200230 & 38.98 & 38.16 & 4.5 & MLT \\
\hline 940823104525 & 44.46 & 43.86 & 4.4 & GNI KIV \\
\hline 940905191318 & 46.24 & 41.94 & 5 & KIV \\
\hline 940905201307 & 46.39 & 42.13 & 4.5 & KIV \\
\hline 940905222806 & 40.27 & 39.53 & 3.4 & KIV \\
\hline 940905222809 & 39.89 & 39.81 & 4 & ERC \\
\hline 940917022441 & 41.48 & 37.88 & 4.9 & MUS \\
\hline 940919152335 & 43.44 & 42.56 & 4.5 & GNI KIV \\
\hline 940921085320 & 43.61 & 42.34 & 4.4 & GNI KIV \\
\hline 941012074401 & 44.41 & 42.4 & 4.7 & GNI KIV \\
\hline 941027163048 & 40.52 & 39.64 & 3.9 & GNI KIV \\
\hline 941028134733 & 40.79 & 39.36 & 3.8 & KIV \\
\hline 941029235256 & 40.72 & 39.41 & 4.2 & GNI KIV \\
\hline 941102123106 & 48.32 & 38.27 & 5 & GNI KIV \\
\hline 941109144607 & 43.12 & 41.88 & 3.7 & GNI KIV \\
\hline 941114075414 & 40.41 & 40.16 & 3.9 & GNI KIV \\
\hline 941204143932 & 43.73 & 42.42 & 2.8 & GNI KIV \\
\hline 941210061503 & 43.65 & 42.5 & 3.1 & GNI KIV \\
\hline 941212131407 & 43.39 & 42.6 & 4.6 & KIV \\
\hline 941213114710 & 43.85 & 41.27 & 3.2 & KIV \\
\hline 941215011720 & 40.47 & 38.84 & 3.9 & KIV \\
\hline 941219150003 & 47.86 & 41.3 & 4.6 & KIV \\
\hline 950104005751 & 48.83 & 41.32 & 3.1 & KIV \\
\hline 950115071330 & 46.9 & 42.92 & 3.8 & KIV \\
\hline 950129041656 & 40.64 & 39.82 & 4.7 & KIV \\
\hline 950129041657 & 40.99 & 39.98 & 4.8 & ERZ ERZ ERZ \\
\hline 950129064245 & 40.13 & 40.01 & 4.1 & KIV \\
\hline 950129133705 & 40.65 & 40.1 & 4 & KIV \\
\hline 950222062942 & 44.86 & 38.58 & 4.9 & KIV \\
\hline 950226113327 & 43.05 & 38.31 & 4 & KIV \\
\hline 950312025633 & 40.11 & 39.67 & 3.6 & GNI KIV \\
\hline 950417130313 & 48.42 & 40.92 & 4 & GNI KIV \\
\hline 950423024054 & 42.25 & 39.43 & 3.7 & GNI KIV \\
\hline 950429153346 & 44.94 & 37.75 & 4.6 & VRN \\
\hline 950513072044 & 50.56 & 40.67 & 4.9 & KIV \\
\hline 950515001654 & 49.43 & 38.48 & 4.7 & KIV \\
\hline 950515002154 & 49.33 & 38.56 & 4.1 & KIV \\
\hline 950516094154 & 42.46 & 39.71 & 4.3 & KIV \\
\hline 950518005206 & 45.64 & 38.38 & 4.2 & KIV \\
\hline 950527212135 & 48.93 & 38.93 & 3.7 & KIV \\
\hline 950605081337 & 44.07 & 42.65 & 3.1 & KIV \\
\hline 950618214222 & 39.85 & 39.9 & 4 & ERC \\
\hline 950626232738 & 48.36 & 39.85 & 4.1 & KIV \\
\hline 950627004646 & 48.41 & 39.9 & 3.6 & KIV \\
\hline 950628092641 & 44.02 & 39.53 & 3.5 & KAP \\
\hline 950701210038 & 41.08 & 39.02 & 3.5 & KIV \\
\hline 950703003417 & 41.35 & 39.08 & 4.3 & KIV \\
\hline
\end{tabular}




\begin{tabular}{|c|c|c|c|c|}
\hline 950703003516 & 41.11 & 39.33 & 4 & KIV \\
\hline 950705142709 & 41.59 & 39.93 & 3.8 & $\mathrm{KIV}$ \\
\hline 950706164511 & 49.4 & 38.6 & 4.1 & KIV \\
\hline 950714140307 & 49 & 42.89 & 4.2 & KIV \\
\hline 950722203708 & 40.23 & 39.92 & 3.6 & KIV \\
\hline 950723181710 & 46.24 & 44.07 & 3.7 & $\mathrm{KIV}$ \\
\hline 950725024929 & 45.97 & 43.72 & 4.1 & $\mathrm{KIV}$ \\
\hline 950802072222 & 43.42 & 42.4 & 3 & KIV \\
\hline 950804132932 & 43.91 & 43.57 & 3.3 & $\mathrm{KIV}$ \\
\hline 950925120244 & 40.87 & 44.66 & 3.3 & $\mathrm{KIV}$ \\
\hline 950925190546 & 45.08 & 40.35 & 3.3 & $\mathrm{KIV}$ \\
\hline 950925191651 & 45.05 & 40.38 & 3 & $\mathrm{KIV}$ \\
\hline 950925201901 & 45.05 & 40.4 & 3 & $\mathrm{KIV}$ \\
\hline 950929095530 & 43.93 & 42.5 & 3 & KIV \\
\hline 951001142001 & 43.6 & 41.9 & 4.5 & KRS \\
\hline 951001142018 & 42.73 & 41.15 & 4.5 & $\mathrm{KIV}$ \\
\hline 951004101244 & 44.38 & 38.48 & 3.6 & ARU NAR \\
\hline 951012191605 & 43.85 & 41.4 & 3 & $\mathrm{KIV}$ \\
\hline 951014104739 & 45.55 & 41.1 & 3.4 & KIV \\
\hline 951014104742 & 46.18 & 41.55 & 3.7 & ARU KAM \\
\hline 951015191054 & 42.25 & 40.88 & 3.7 & NAR PAR \\
\hline 951015191104 & 42.4 & 41.03 & 3.9 & KIV \\
\hline 951021122900 & 42.25 & 40.42 & 3.7 & KAM \\
\hline 951101041618 & 47.95 & 41.78 & 3.9 & KIV \\
\hline 951104232347 & 49.27 & 38.98 & 3.9 & $\mathrm{KIV}$ \\
\hline 951108010006 & 43.68 & 42.32 & 3 & KIV \\
\hline 951201150913 & 45.6 & 42.3 & 3.2 & KIV \\
\hline 951203181212 & 43.17 & 43.15 & 3.1 & KIV \\
\hline 951205184932 & -12345 & -12345 & 5.8 & ELZ ELZ ELZ ERC ERZ ERZ ERZ KIV MUS \\
\hline 951205185238 & 40.26 & 39.6 & 5.3 & $\mathrm{KIV}$ \\
\hline 951205185240 & -12345 & -12345 & 12345 & ELZ ELZ ELZ ERC \\
\hline 951206074948 & 40.11 & 39.55 & 4.4 & KIV \\
\hline 951206074951 & 39.64 & 39.09 & 4.4 & ERC \\
\hline 951206164602 & 43.6 & 42.53 & 3 & KIV \\
\hline 951209230903 & 43.38 & 42.34 & 3.7 & KIV \\
\hline 951211070034 & 40.16 & 39.38 & 4 & $\mathrm{KIV}$ \\
\hline 951218085615 & 40.35 & 39.65 & 3.8 & KIV \\
\hline 951220073108 & 40.11 & 39.55 & 3.8 & $\mathrm{KIV}$ \\
\hline 951228163831 & 49.28 & 40.2 & 4.2 & KIV \\
\hline 951229014814 & 48.52 & 39.55 & 3.5 & $\mathrm{KIV}$ \\
\hline 951229032021 & 42.59 & 38.91 & 3.9 & LER VNN \\
\hline 960103084226 & 48.7 & 38.96 & 4.9 & KIV \\
\hline 960103120738 & 45.88 & 42.93 & 3.1 & KIV \\
\hline 960106005655 & 50.28 & 40.97 & 3.9 & KIV \\
\hline 960112032244 & 41.84 & 40.27 & 4.1 & $\mathrm{KIV}$ \\
\hline 960112043839 & 46.35 & 40.25 & 3.7 & PAR \\
\hline 960114151811 & 40.17 & 39.21 & 4.7 & KIV \\
\hline 960120235625 & 45.55 & 40.4 & 4.4 & LER \\
\hline 960120235629 & 45.85 & 40.59 & 4.3 & KIV \\
\hline 960129190352 & 45.32 & 41.45 & 3 & KIV \\
\hline 960205044540 & 46.19 & 42.92 & 3.8 & $\mathrm{KIV}$ \\
\hline 960207122703 & 39.31 & 38.43 & 4.7 & ELZ ELZ ELZ \\
\hline
\end{tabular}




\begin{tabular}{|c|c|c|c|c|}
\hline 960214074318 & 39.23 & 39.61 & 4 & ERC \\
\hline 960217054246 & 42.79 & 43.69 & 3.8 & KIV \\
\hline 960301181748 & 44.55 & 38.96 & 3.6 & KAP \\
\hline 960305093716 & 42.2 & 42.37 & 3 & KIV \\
\hline 960306144905 & 42.5 & 40.52 & 4.1 & KIV \\
\hline 960308120639 & 47.36 & 42.72 & 3.6 & KIV \\
\hline 960308173015 & 40.99 & 39.29 & 3.7 & KIV \\
\hline 960313040421 & 42.47 & 42.05 & 3 & KIV \\
\hline 960313121354 & 44.64 & 38.43 & 3.5 & KIV \\
\hline 960314103234 & 43.32 & 38.52 & 3.7 & VNN \\
\hline 960320095039 & 46.81 & 41.32 & 3.8 & KAP \\
\hline 960321111804 & 40.12 & 39.81 & 4.3 & KIV \\
\hline 960329160809 & 47.65 & 40.61 & 3.9 & KIV \\
\hline 960403004916 & 50.11 & 41.34 & 3.7 & KIV \\
\hline 960406185700 & 40.19 & 39.58 & 3.7 & KIV \\
\hline 960413010704 & 43.42 & 42.53 & 3 & KIV \\
\hline 960415045604 & 44.27 & 41.3 & 3.5 & KIV \\
\hline 960421141356 & 40.33 & 38.54 & 4.6 & KIV \\
\hline 960422144237 & 47.33 & 39.19 & 5.6 & KIV PAR \\
\hline 960422234717 & 40.46 & 38.86 & 4.1 & KIV \\
\hline 960425202941 & 42.39 & 40.14 & 3.9 & KIV \\
\hline 960501033123 & 49.14 & 40.26 & 3.8 & KIV \\
\hline 960501175739 & 43.97 & 41.23 & 3.2 & KIV \\
\hline 960504081919 & 38.82 & 42.33 & 5.4 & KAP VNN \\
\hline 960504081942 & 41.09 & 39.56 & 3.7 & KIV \\
\hline 960507233049 & 44.32 & 41.7 & 4 & HAR \\
\hline 960508062025 & 46.02 & 40.63 & 3.3 & KIV \\
\hline 960509074448 & 48.88 & 40.98 & 3.6 & KIV \\
\hline 960509105845 & 48.33 & 40.71 & 4.1 & KIV \\
\hline 960518074728 & 42.5 & 42.6 & 3 & KIV \\
\hline 960519042421 & 45.21 & 41.75 & 3.7 & KIV \\
\hline 960524093038 & 42.53 & 41.77 & 3 & KIV \\
\hline 960528045010 & 42.06 & 41.97 & 4.9 & KIV \\
\hline 960528050748 & 42.23 & 42 & 3 & KIV \\
\hline 960529120330 & 42.18 & 41.92 & 3 & KIV \\
\hline 960529222900 & 43.92 & 41.08 & 3 & KIV \\
\hline 960531092544 & 47.11 & 43.35 & 4.2 & KIV \\
\hline 960602131200 & -12345 & -12345 & 12345 & SBVR \\
\hline 960602131319 & 43.99 & 41.07 & 3.7 & VNN \\
\hline 960602131324 & 44.16 & 41.31 & 3.5 & KIV \\
\hline 960602153029 & 40.92 & 43.34 & 3.5 & KIV \\
\hline 960602162105 & 43.88 & 41.02 & 3 & KIV \\
\hline 960602214322 & 43.87 & 41.05 & 3 & KIV \\
\hline 960605221137 & 42.4 & 39.65 & 3.8 & KIV \\
\hline 960606052331 & 43.67 & 42.48 & 3.2 & KIV \\
\hline 960606052333 & 43.8 & 42.49 & 4.6 & HAR \\
\hline 960608231206 & 40.26 & 39.22 & 3.3 & KIV \\
\hline 960609021654 & 44.04 & 41.11 & 4.1 & AMB HNA \\
\hline 960609021659 & 44.01 & 41.37 & 4 & KIV SBVR \\
\hline 960609022017 & 43.98 & 41.05 & 3 & KIV \\
\hline 960609051821 & -12345 & -12345 & 12345 & SBVR SGMI SPIK \\
\hline 960609051918 & 43.62 & 40.82 & 4 & LER VNN \\
\hline
\end{tabular}




\begin{tabular}{|c|c|c|c|c|}
\hline 960609192000 & 43.87 & 41.03 & 4.1 & KIV \\
\hline 960609200944 & 42.2 & 41.93 & 3 & KIV \\
\hline 960609202553 & 48.45 & 39.97 & 3.7 & KIV \\
\hline 960609215847 & 42.18 & 41.97 & 3.1 & KIV \\
\hline 960617132547 & 42.18 & 41.97 & 3.3 & KIV \\
\hline 960617215436 & 42.57 & 38.8 & 2.4 & KIV \\
\hline 960618081056 & 45.59 & 38.74 & 4.9 & KAP NAR \\
\hline 960618081105 & 45.78 & 39.14 & 4.5 & KIV \\
\hline 960619070758 & 45.6 & 39.17 & 4 & AMB KAM \\
\hline 960619070801 & 45.86 & 38.9 & 3.9 & KIV \\
\hline 960623085454 & 40.48 & 39.41 & 4.3 & KIV \\
\hline 960628113046 & 42.43 & 38.04 & 3 & KIV \\
\hline 960629012217 & 43.98 & 41.3 & 3 & KIV \\
\hline 960630073958 & 39.07 & 39.93 & 4.9 & AMB \\
\hline 960630074003 & 40.55 & 39.13 & 3.9 & KIV \\
\hline 960702090213 & 42.97 & 38.35 & 3.8 & AMB \\
\hline 960702090218 & 43.05 & 38.67 & 3.5 & KIV \\
\hline 960706201721 & 47.04 & 42.53 & 3.6 & KIV \\
\hline 960708031603 & 47.16 & 43.15 & 3.7 & KIV \\
\hline 960725182529 & 40.51 & 39.92 & 4 & KIV \\
\hline 960806115251 & 43.65 & 42.47 & 3 & KIV \\
\hline 960806165641 & 48.38 & 41.37 & 3.8 & KIV \\
\hline 960827001649 & 42.65 & 40.31 & 4 & ARU \\
\hline 960827001654 & 43.22 & 40.08 & 3.2 & KIV \\
\hline 960903231103 & 49.45 & 40.21 & 4 & KIV \\
\hline 960908202622 & 45.33 & 42.33 & 3 & KIV \\
\hline 960910034056 & 45.4 & 42.25 & 3 & KIV \\
\hline 960912084927 & 40.31 & 39.38 & 3.3 & KIV \\
\hline 960918051641 & 41.36 & 39.64 & 4.1 & KIV \\
\hline 960928121054 & 50.43 & 41.06 & 4.4 & KIV \\
\hline 961004084304 & 43.75 & 42.47 & 3.5 & KIV \\
\hline 961005053233 & 44.4 & 39.34 & 3.5 & ARU \\
\hline 961009215151 & 40.13 & 39.89 & 3.7 & KIV \\
\hline 961012212531 & 43.68 & 42.48 & 3 & KIV \\
\hline 961013001008 & 44.61 & 38.4 & 4.1 & AMB HAR \\
\hline 961013214900 & 45.83 & 44.44 & 3.7 & KIV \\
\hline 961013225049 & 40.02 & 43.52 & 3.7 & KIV \\
\hline 961106134908 & 40.4 & 38.71 & 3.8 & KIV \\
\hline 961110005724 & 42.5 & 42.03 & 3 & KIV \\
\hline 961115012335 & 42.65 & 42.43 & 3.1 & KIV \\
\hline 961115012353 & 42.97 & 42.09 & 4.2 & AMB \\
\hline 961127160843 & 49.25 & 42.18 & 3.8 & KIV \\
\hline 961129224006 & 40.7 & 39.4 & 3.6 & KIV \\
\hline 961209065608 & 50.68 & 40.94 & 3.4 & GNI KIV \\
\hline 961214002635 & 48.41 & 40.72 & 3.8 & KIV \\
\hline 961220080903 & 41.02 & 38.93 & 4.5 & KIV \\
\hline 961220114228 & 45.75 & 42.27 & 3 & KIV \\
\hline 961220114232 & 45.28 & 42 & 3.8 & NAR \\
\hline 961220170227 & 44.49 & 41.38 & 3.5 & HNA \\
\hline 961220170228 & 44.61 & 41.61 & 3.9 & KIV \\
\hline 961222184047 & 44.01 & 41.28 & 3 & KIV \\
\hline 961225150233 & 48.13 & 42.46 & 3.9 & KIV \\
\hline
\end{tabular}




\begin{tabular}{|c|c|c|c|c|}
\hline 961225224823 & 40.7 & 38.59 & 4.3 & KIV \\
\hline 970121231546 & 43.97 & 41.18 & 3.5 & AMB \\
\hline 970128105654 & 48.56 & 42.51 & 4.8 & KIV \\
\hline 970129064343 & 47.9 & 42.57 & 3.7 & KIV \\
\hline 970206160345 & 47.19 & 43.04 & 4 & KIV \\
\hline 970209214900 & -12345 & -12345 & 12345 & SBVR SGMI SPIK STPV SVNZ SVNZ SVNZ \\
\hline 970209214907 & 43.93 & 41.41 & 4.4 & LER \\
\hline 970209214909 & 43.95 & 41.46 & 4.3 & KIV \\
\hline 970213004738 & 48.8 & 38.69 & 4.6 & GNI KIV \\
\hline 970215014053 & 40.35 & 38.3 & 4.2 & GNI KIV \\
\hline 970215072619 & 40.33 & 43.06 & 3.8 & GNI KIV \\
\hline 970216105730 & 46.05 & 41.99 & 3.4 & GNI KIV \\
\hline 970216105731 & 46.28 & 41.95 & 3.6 & HAR \\
\hline 970228125722 & 48.04 & 38.09 & 5.7 & KIV \\
\hline 970228125728 & 48.33 & 38.07 & 6.1 & AMB KAP NPP PAR VNN \\
\hline 970228135603 & 47.93 & 38.22 & 4.5 & KIV \\
\hline 970228135605 & 48.87 & 38.32 & 4.8 & AMB \\
\hline 970228163549 & 48.87 & 38.32 & 4.2 & KAP \\
\hline 970228205514 & 47.92 & 38.09 & 4.5 & KIV \\
\hline 970228205515 & 48.87 & 38.32 & 4.7 & PAR \\
\hline 970228214622 & 47.98 & 38.16 & 4.6 & GNI KIV \\
\hline 970228214627 & 48.87 & 38.32 & 4.7 & PAR \\
\hline 970301130929 & 44.48 & 40.12 & 3.7 & KAP \\
\hline 970302023448 & 48.5 & 37.72 & 4.2 & PAR \\
\hline 970302023537 & 41.07 & 40.35 & 3.3 & GNI KIV \\
\hline 970302182940 & 47.25 & 37.67 & 5.7 & AMB KAP NPP PAR VNN \\
\hline 970303195023 & 48.87 & 38.32 & 4.2 & KAP \\
\hline 970304142250 & 41.42 & 39.38 & 4.3 & KAP \\
\hline 970304142300 & 40.72 & 39.29 & 4.2 & GNI KIV \\
\hline 970305070639 & 42.49 & 37.94 & 4.3 & KAP \\
\hline 970305165842 & 49.45 & 40.93 & 4.6 & GNI KIV \\
\hline 970306232834 & 43.58 & 38.75 & 3.7 & PAR \\
\hline 970306232837 & 43.66 & 39.11 & 3.6 & GNI KIV \\
\hline 970318035039 & 42.12 & 39.72 & 3.7 & GNI KIV \\
\hline 970321230041 & 48.17 & 38.42 & 4.2 & LER \\
\hline 970402012536 & 43.8 & 42.4 & 3.6 & LER \\
\hline 970408054413 & 48.08 & 38.15 & 4.2 & GNI KIV \\
\hline 970419110048 & 42.27 & 39.03 & 4.2 & KIV \\
\hline 970420180430 & 48.06 & 38.43 & 3.8 & KIV \\
\hline 970424085605 & 43.99 & 42.84 & 4 & HAR KAP \\
\hline 970424085606 & 43.31 & 42.28 & 3.3 & KIV \\
\hline 970426054215 & 45.59 & 38.88 & 3.6 & $\mathrm{KIV}$ \\
\hline 970510175720 & 46.94 & 40.26 & 4.1 & KAP \\
\hline 970510175724 & 46.39 & 39.92 & 3.8 & KIV \\
\hline 970514012156 & 45.17 & 38.3 & 3.4 & KIV \\
\hline 970514012157 & 45.37 & 38.75 & 4 & HAR PAR \\
\hline 970524025919 & 43.17 & 42.05 & 3.6 & VNN \\
\hline 970528050421 & 48.51 & 38.73 & 4 & GNI KIV \\
\hline 970529234612 & 41.52 & 38.04 & 3.5 & GNI KIV \\
\hline 970531144843 & 44.03 & 41.13 & 3.7 & KAP LER \\
\hline 970601103338 & 42.05 & 39.9 & 3.9 & GNI KIV \\
\hline 970613211019 & 40.09 & 44.06 & 4 & GNI KIV \\
\hline
\end{tabular}




\begin{tabular}{|c|c|c|c|c|}
\hline 970622004634 & 49.28 & 39.57 & 3.7 & GNI KIV \\
\hline 970627073539 & 49.69 & 43 & 4.1 & GNI KIV \\
\hline 970628064501 & 48.22 & 41.77 & 3.9 & GNI KIV \\
\hline 970628124915 & -12345 & -12345 & 0 & SPIK STPV \\
\hline 970702144759 & -12345 & -12345 & 12345 & SPIK STPV \\
\hline 970708061121 & 48.65 & 40.76 & 4.2 & GNI KIV \\
\hline 970708062751 & 48.77 & 40.7 & 3.6 & KAP \\
\hline 970710155041 & 40.45 & 39.01 & 3.7 & GNI KIV \\
\hline 970712220213 & 46.02 & 43.05 & 3.5 & GNI KIV \\
\hline 970713194116 & 43.27 & 38.24 & 3.7 & GNI KIV \\
\hline 970715135842 & 48.81 & 40.56 & 4 & GNI KIV \\
\hline 970718073353 & 45.11 & 41.1 & 4.1 & KIV \\
\hline 970718073401 & 44.96 & 41.13 & 4.4 & ARU LER \\
\hline 970802114217 & 48.18 & 40.75 & 3.9 & KIV \\
\hline 970808023853 & 40.85 & 39.93 & 4.5 & ARU LER \\
\hline 970808023903 & 41.96 & 39.84 & 4.6 & KIV \\
\hline 970825024803 & 44.11 & 39.34 & 3.9 & ARU LER \\
\hline 970825024807 & 44.1 & 39.23 & 4.4 & GNI KIV \\
\hline 970825192149 & 45.7 & 41.41 & 3.6 & ARU LER \\
\hline 970826224444 & 42.62 & 40.22 & 3.5 & ARU \\
\hline 970826224445 & 43.14 & 40.07 & 3.5 & GNI KIV \\
\hline 970828014314 & 40.88 & 39.14 & 3.5 & GNI KIV \\
\hline 970828201217 & 40.94 & 39.53 & 3.6 & GNI KIV \\
\hline 970831032633 & 41.74 & 39.71 & 3.3 & GNI KIV \\
\hline 970909173452 & 42.03 & 44.58 & 4 & GNI KIV \\
\hline 970920234219 & 40.17 & 38.85 & 3.8 & GNI KIV \\
\hline 970926111252 & 39.92 & 39.95 & 4.6 & ARU \\
\hline 970926111311 & 41.34 & 39.23 & 3.7 & GNI KIV \\
\hline 971001121114 & 42.49 & 39.8 & 2.9 & GNI KIV \\
\hline 971001131232 & 43.22 & 38.75 & 3.2 & GNI KIV \\
\hline 971001135320 & 43.2 & 38.73 & 3 & GNI KIV \\
\hline 971002010630 & 44.42 & 38.83 & 4.1 & ARU \\
\hline 971002010636 & 44.27 & 38.98 & 4.4 & GNI KIV \\
\hline 971003084019 & 40.09 & 39.44 & 2.9 & GNI KIV \\
\hline 971003215507 & 48.59 & 40.68 & 4.7 & GNI KIV \\
\hline 971009182859 & 48.31 & 41.25 & 3.7 & GNI KIV \\
\hline 971009184722 & 41.33 & 39.12 & 3.1 & GNI KIV \\
\hline 971010212045 & 44.63 & 38.16 & 4 & AMB \\
\hline 971010212058 & 43.71 & 38.6 & 4 & GNI KIV \\
\hline 971010220507 & 43.96 & 41.38 & 3.9 & ARU KAM \\
\hline 971010233529 & 44.58 & 38.2 & 4.2 & ARU \\
\hline 971010233534 & 44.31 & 38.54 & 4 & GNI KIV \\
\hline 971012050604 & 43.16 & 38.57 & 4.1 & ARU \\
\hline 971012050611 & 43.28 & 38.53 & 4.2 & GNI KIV \\
\hline 971017211408 & 47.85 & 38.27 & 3.6 & GNI KIV \\
\hline 971019075103 & 48.85 & 40.8 & 4.1 & GNI KIV \\
\hline 971022232853 & 42.64 & 38.04 & 3.7 & GNI KIV \\
\hline 971023160637 & 41.33 & 38.71 & 4 & GNI KIV \\
\hline 971030040532 & 43.32 & 38.57 & 4.1 & ARU HNA \\
\hline 971030040534 & 43.53 & 38.76 & 4.4 & KIV \\
\hline 971102002555 & 43.5 & 38.6 & 4 & ARU HNA \\
\hline 971102002558 & 43.34 & 38.77 & 4.4 & KIV MUR \\
\hline
\end{tabular}




\begin{tabular}{|c|c|c|c|c|}
\hline 971102221126 & 42 & 40.25 & 4.3 & HNA \\
\hline 971102221136 & 42.25 & 40.23 & 3.7 & KIV \\
\hline 971103000758 & -12345 & -12345 & 12345 & MLZ MLZ MLZ \\
\hline 971103080753 & 41.6 & 39.3 & 4.3 & $\mathrm{ARU}$ \\
\hline 971103080756 & 42.48 & 38.77 & 4 & KIV \\
\hline 971103080757 & 42.4 & 38.76 & 4.9 & MLZ MLZ MLZ \\
\hline 971103084651 & 42.28 & 38.81 & 4.5 & KIV \\
\hline 971103145107 & 42.73 & 38.83 & 3.6 & ARU \\
\hline 971103145110 & 42.31 & 38.79 & 4.1 & KIV \\
\hline 971104041608 & 42.4 & 38.67 & 3.9 & VNN \\
\hline 971104041609 & 42.39 & 38.58 & 3.6 & KIV \\
\hline 971105031515 & 42.97 & 38.27 & 4 & KIV \\
\hline 971108180135 & 40.47 & 39.38 & 3.2 & KIV \\
\hline 971109052713 & 48.55 & 41.83 & 3.6 & $\mathrm{ARU}$ \\
\hline 971109052715 & 48.73 & 42.05 & 4.5 & KIV \\
\hline 971114164345 & 41.82 & 39.81 & 3.3 & KIV \\
\hline 971127173427 & -12345 & -12345 & 5.2 & SPIK STPV \\
\hline 971127173429 & 45.37 & 41.86 & 3.5 & KIV \\
\hline 971127173430 & 45.38 & 41.67 & 5 & GYM HAR HNA KAM LER NAR TCH TSO \\
\hline 971128100710 & 45.22 & 41.62 & 3.6 & KAP \\
\hline 971202155304 & 49.14 & 40.34 & 4 & KIV \\
\hline 971204060618 & 47.89 & 41.68 & 4.3 & KIV \\
\hline 971204060620 & 47.58 & 41.17 & 4.1 & AMB \\
\hline 971209031609 & 41.17 & 37.83 & 4.6 & PAR \\
\hline 971211100715 & 48.58 & 40.7 & 4 & KIV \\
\hline 971212080118 & 41.93 & 39.76 & 4 & KIV \\
\hline 971217202113 & 48.91 & 40.62 & 3.7 & KIV \\
\hline 971228055951 & 40.56 & 40.04 & 3.3 & KIV \\
\hline 971230210907 & 46.29 & 41.9 & 3.5 & LER \\
\hline 980107193335 & 40.66 & 38.56 & 2.9 & KIV \\
\hline 980109141603 & 48.92 & 40.4 & 4.3 & KIV \\
\hline 980110190738 & 42.21 & 40.41 & 2.9 & KIV \\
\hline 980112123634 & 41.65 & 39.92 & 3.5 & KIV \\
\hline 980116223635 & 40.44 & 39.69 & 3.3 & KIV \\
\hline 980119200214 & 43.9 & 39.36 & 3.3 & KIV \\
\hline 980121055528 & 47.3 & 42.97 & 3.9 & KIV \\
\hline 980123123251 & 43.99 & 39.34 & 4.2 & KIV \\
\hline 980128113704 & 42.44 & 38.97 & 3.2 & KIV \\
\hline 980204233055 & 47.77 & 43.1 & 3.7 & KIV \\
\hline 980223021921 & 45.71 & 43.21 & 3.5 & KIV \\
\hline 980224064604 & 41.31 & 39.16 & 3.7 & KIV \\
\hline 980227180243 & 49.12 & 39.65 & 4.6 & KIV \\
\hline 980302021206 & 41.66 & 39.77 & 3.3 & KIV \\
\hline 980303213942 & 46.96 & 40.51 & 3.7 & LER \\
\hline 980303213944 & 46.72 & 40.25 & 4.5 & KIV \\
\hline 980318211137 & 48.52 & 41.08 & 3.5 & KIV \\
\hline 980325094422 & 43.06 & 38.4 & 3.1 & KIV \\
\hline 980325105157 & 46 & 42.9 & 4.5 & KIV \\
\hline 980325180931 & 43.66 & 38.67 & 3.2 & KIV \\
\hline 980411094852 & 46 & 41.46 & 3.5 & KIV \\
\hline 980411094900 & 45.6 & 41.52 & 3.6 & LER \\
\hline 980413151430 & 41.07 & 39.23 & 5 & BNG ERZ ERZ ERZ MUS SLH TER \\
\hline
\end{tabular}




\begin{tabular}{|c|c|c|c|c|}
\hline 980413151431 & 41.07 & 39.31 & 4.6 & KIV \\
\hline 980413151446 & -12345 & -12345 & 5.2 & BIN ERZ ERZ ERZ MUS SLH TER \\
\hline 980413195627 & 42.02 & 39.94 & 4.4 & KIV \\
\hline 980414165426 & 41.38 & 39.01 & 3.3 & KIV \\
\hline 980414170043 & 41.2 & 39.17 & 3.3 & KIV \\
\hline 980418105715 & 41.03 & 39.92 & 3.5 & KIV \\
\hline 980418194700 & 49.9 & 40.92 & 4.6 & KIV \\
\hline 980420160423 & 40.92 & 39.3 & 3.4 & KIV \\
\hline 980424043744 & 41.66 & 39.24 & 3 & KIV \\
\hline 980424220008 & 47.16 & 39.9 & 4 & KIV \\
\hline 980427024925 & 40.47 & 39.91 & 3.8 & KIV \\
\hline 980428035157 & 40.4 & 38.69 & 4.3 & KIV \\
\hline 980428202819 & 42.13 & 39.42 & 4.4 & KAM \\
\hline 980428202825 & 42.56 & 39.58 & 3.9 & KIV \\
\hline 980501133934 & 41.9 & 41.01 & 4.1 & LER \\
\hline 980501133939 & 42.08 & 40.03 & 4.1 & KIV \\
\hline 980502111326 & 45.29 & 42.54 & 4 & KIV \\
\hline 980504010254 & 49.83 & 41.16 & 4.2 & KIV \\
\hline 980511002536 & 42.32 & 39.54 & 4.1 & LER \\
\hline 980511002544 & 42.32 & 39.73 & 4 & KIV \\
\hline 980511050731 & 49.09 & 40.26 & 3.8 & KIV \\
\hline 980511072501 & 46.3 & 42.9 & 4 & KIV \\
\hline 980514143111 & 47.03 & 42.87 & 4 & KIV \\
\hline 980515081648 & 41.13 & 39.9 & 3.7 & KAM \\
\hline 980515081653 & 41.77 & 39.81 & 3.4 & KIV \\
\hline 980516124607 & 46 & 42.2 & 4 & KIV \\
\hline 980517152651 & 42.38 & 38.72 & 3.2 & KIV \\
\hline 980517211008 & 42.77 & 39.74 & 3.3 & KIV \\
\hline 980523204955 & 46.79 & 43.08 & 4 & KIV \\
\hline 980524112017 & 45.27 & 42.63 & 4 & KIV \\
\hline 980525152720 & 47.06 & 43.01 & 4 & KIV \\
\hline 980527023405 & 48.09 & 40.51 & 4 & KIV \\
\hline 980527062933 & 44.07 & 38.1 & 3.8 & AMB \\
\hline 980528072727 & 44.69 & 38.28 & 3.7 & AMB \\
\hline 980528083453 & 44.79 & 43.58 & 4 & KIV \\
\hline 980528084108 & 45.13 & 43.67 & 4 & KIV \\
\hline 980528085515 & 45.18 & 43.56 & 4 & KIV \\
\hline 980528142126 & 41.84 & 39.86 & 3.1 & KIV \\
\hline 980529201003 & 40.63 & 38.69 & 3.9 & KIV \\
\hline 980530114555 & 44.99 & 43.25 & 4 & KIV \\
\hline 980602105318 & 47.92 & 42.01 & 4 & KIV \\
\hline 980603175625 & 40.69 & 39.63 & 3.4 & KIV \\
\hline 980608095515 & 43.69 & 42.29 & 4.7 & KIV \\
\hline 980608095519 & 44.33 & 42.17 & 4 & PAR \\
\hline 980615201814 & 42.97 & 44.02 & 4 & KIV \\
\hline 980618065154 & 50.39 & 41.45 & 4 & KIV \\
\hline 980623040228 & 40.51 & 40.07 & 3.9 & KIV \\
\hline 980624225501 & 45.3 & 42.1 & 4 & KIV \\
\hline 980625230436 & 47.5 & 43.2 & 4 & KIV \\
\hline 980707112811 & 46.04 & 41.82 & 3.6 & AMB \\
\hline 980707112818 & 46.08 & 42.07 & 4.2 & KIV \\
\hline 980709141919 & 48.55 & 38.58 & 6.2 & AMB ARU KAP NPP PAR VNN VRN \\
\hline
\end{tabular}




\begin{tabular}{|c|c|c|c|c|}
\hline 980709141921 & 48.48 & 38.69 & 5.8 & KIV \\
\hline 980709162336 & 48.62 & 38.74 & 4.1 & KIV \\
\hline 980709162433 & 48.21 & 37.28 & 5.1 & AMB ARU KAP PAR \\
\hline 980717045205 & 49.83 & 38.17 & 3.5 & KIV \\
\hline 980718115416 & 45.76 & 44.26 & 3.8 & KIV \\
\hline 980719060655 & 45.04 & 42.76 & 4.9 & KIV \\
\hline 980724070841 & 42.94 & 38.91 & 3.2 & KIV \\
\hline 980731164042 & 42.84 & 38.57 & 3.5 & KIV \\
\hline 980803004924 & 45.87 & 38.67 & 3.8 & KAP \\
\hline 980807172650 & 44.02 & 41.29 & 3.5 & ARU \\
\hline 980814235805 & 49.57 & 41.23 & 3.9 & KIV \\
\hline 980819203228 & 42.76 & 39.11 & 3.5 & ARU \\
\hline 980920194721 & 46.31 & 42.45 & 4.4 & KIV \\
\hline 980925135926 & 44.15 & 39.64 & 3.7 & ARU \\
\hline 980925135936 & 44.22 & 39.6 & 4.5 & KIV \\
\hline 980926041149 & 40.21 & 39.31 & 3.8 & KIV \\
\hline 980926054435 & 43.68 & 39.05 & 3 & KIV \\
\hline 980929150811 & 43.75 & 38.57 & 3.7 & KIV \\
\hline 981008204809 & 40.34 & 38.72 & 4.4 & KIV \\
\hline 981008211843 & 44.19 & 44.05 & 3.8 & KIV \\
\hline 981011072225 & 42.72 & 38.95 & 3.8 & KIV \\
\hline 981011072246 & 41.93 & 39.07 & 4 & AKS \\
\hline 981012075622 & 42.32 & 40.24 & 3.6 & AKS HAR \\
\hline 981012075623 & 42.48 & 40.67 & 3.8 & KIV \\
\hline 981012183226 & 49.22 & 40.04 & 4.1 & KIV \\
\hline 981015122656 & 40.49 & 38.99 & 3.6 & KIV \\
\hline 981016053816 & 40.26 & 39.15 & 3.2 & KIV \\
\hline 981018040400 & -12345 & -12345 & 0 & STPV \\
\hline 981020074416 & 40.37 & 39.64 & 3.7 & KIV \\
\hline 981024212748 & 43.08 & 38.65 & 3.6 & KIV \\
\hline 981025222143 & 44.38 & 40.75 & 3.7 & AKS TSO \\
\hline 981025222144 & -12345 & -12345 & 12345 & SPIK STPV SVNZ SVNZ SVNZ \\
\hline 981025222153 & 44.21 & 40.87 & 3.4 & KIV \\
\hline 981106041454 & 41.6 & 39.6 & 4 & AKS KIV \\
\hline 981106103221 & 40.26 & 39.11 & 4.1 & KIV \\
\hline 981109123002 & 43.32 & 39.74 & 2.9 & KIV \\
\hline 981109213644 & 45.14 & 38.08 & 4 & KIV \\
\hline 981109213645 & 45.24 & 38.51 & 4 & ARU \\
\hline 981110052019 & 40.14 & 38.76 & 3.6 & KIV \\
\hline 981110053933 & 40.29 & 39.15 & 4.5 & KIV \\
\hline 981110053934 & 40.67 & 39.33 & 3.8 & AKS \\
\hline 981110084234 & 40.01 & 39.19 & 4.6 & KIV \\
\hline 981110085927 & 40.06 & 39.2 & 4 & KIV \\
\hline 981110155454 & 40.32 & 39.15 & 4.1 & $\mathrm{KIV}$ \\
\hline 981111030830 & 40.19 & 39.18 & 3.5 & KIV \\
\hline 981113040033 & 46.5 & 42.88 & 3.7 & KIV \\
\hline 981118053227 & 40.02 & 39.06 & 3.8 & $\mathrm{KIV}$ \\
\hline 981118113720 & 45.12 & 38.55 & 4.2 & AKS KIV TSO \\
\hline 981118113950 & 40.19 & 39.35 & 3.7 & KIV \\
\hline 981118115206 & 45.12 & 38.55 & 4.2 & ARU \\
\hline 981118131710 & 40.27 & 38.96 & 3.5 & KIV \\
\hline 981118162702 & 45.1 & 38.24 & 3.9 & $\mathrm{AMB}$ \\
\hline
\end{tabular}




\begin{tabular}{|c|c|c|c|c|}
\hline 981118162703 & 45.44 & 38.52 & 3.7 & KIV \\
\hline 981118165836 & 40.45 & 39.17 & 3.9 & KIV \\
\hline 981118170236 & 40.07 & 39.03 & 3.5 & KIV \\
\hline 981119020306 & 40.09 & 39.01 & 3.1 & KIV \\
\hline 981119175354 & 45.32 & 38.5 & 3.7 & AMB \\
\hline 981121060843 & 40.1 & 39.06 & 3 & KIV \\
\hline 981123111133 & 45.7 & 38.18 & 4.7 & AKS \\
\hline 981123111138 & 45.14 & 38.35 & 4.6 & KIV \\
\hline 981208231311 & 43.76 & 42.57 & 3.9 & AMB \\
\hline 981213182314 & 45.96 & 42.26 & 3.9 & PAR \\
\hline 981213182317 & 45.91 & 42.18 & 4 & KIV \\
\hline 981219161515 & 41.97 & 40.04 & 4.4 & HAR \\
\hline 981219161520 & 42.02 & 40.06 & 4.7 & KIV \\
\hline 981219182256 & 40.94 & 39.23 & 3.3 & KIV \\
\hline 981219230956 & 41.45 & 40.25 & 3.9 & AMB \\
\hline 981219231001 & 42.28 & 40.14 & 3.9 & KIV \\
\hline 981220032115 & 40.01 & 38.58 & 4.1 & KIV \\
\hline 981220134842 & 40.07 & 39.4 & 4.2 & KIV \\
\hline 981221112401 & 45.58 & 39.41 & 3.5 & AKS \\
\hline 981223162055 & 42.22 & 40.9 & 5 & HAR HNA KAM LER TCH TSO \\
\hline 981223162059 & 42.53 & 40.79 & 4.8 & KIV \\
\hline 981223170118 & 46.16 & 41.95 & 4 & KIV \\
\hline 981228062532 & 40.61 & 38.79 & 3.6 & KIV \\
\hline 981229174020 & 41.54 & 43.25 & 4.2 & KIV \\
\hline 981231065520 & 45.83 & 38.48 & 3.9 & AMB \\
\hline 990105201939 & 46.11 & 41.96 & 4.1 & AKS \\
\hline 990105201942 & 46.04 & 42.06 & 4.1 & KIV \\
\hline 990113172319 & 40.4 & 38.25 & 3.2 & KIV \\
\hline 990114070139 & 40.43 & 39.2 & 3.7 & KIV \\
\hline 990114224516 & 44.03 & 41.31 & 4.3 & ARU KIV SBVR SGMI SNIT SPIK STBO STPV SVNZ SVNZ SVNZ \\
\hline 990115114747 & 40.28 & 38.42 & 3.7 & KIV \\
\hline 990115193547 & 43.6 & 43.56 & 4 & KIV \\
\hline 990119102532 & -12345 & -12345 & 0 & SBVR \\
\hline 990121082139 & 48.84 & 41.18 & 4 & KIV \\
\hline 990123234209 & 44.15 & 38.34 & 3.9 & KAP \\
\hline 990125230709 & 40.85 & 38.36 & 3.7 & KIV \\
\hline 990126234931 & 48.1 & 42.24 & 4.6 & KAP \\
\hline 990126234932 & 47.94 & 42.1 & 4.5 & KIV \\
\hline 990128104323 & 40.31 & 38.62 & 3.2 & KIV \\
\hline 990129074729 & 41.59 & 38.77 & 3.9 & KAP KIV \\
\hline 990131050700 & 48.26 & 43.62 & 5.7 & AKS AMB ARU HAR HNA KAM KAP LER PAR TCH TSO VNN VRN \\
\hline 990131050714 & 46.9 & 43.25 & 6.1 & KIV \\
\hline 990131053607 & 47.26 & 42.78 & 4 & KIV \\
\hline 990131060729 & 47.11 & 42.9 & 4 & KIV \\
\hline 990131063106 & 47.09 & 43.27 & 4.2 & KIV \\
\hline 990131072308 & 47 & 42.99 & 4 & KIV \\
\hline 990131075252 & 47 & 43.03 & 4 & KIV \\
\hline 990131081555 & 46.95 & 43.96 & 5.1 & AKS AMB ARU KAP PAR VNN \\
\hline 990131081603 & 47.01 & 43.07 & 4.2 & KIV \\
\hline 990131083727 & 47.02 & 42.98 & 4 & KIV \\
\hline 990131105311 & 46.89 & 42.82 & 4 & KIV \\
\hline 990131114010 & 47.05 & 43.15 & 3.9 & KIV \\
\hline
\end{tabular}




\begin{tabular}{|c|c|c|c|c|}
\hline 990131120609 & 47.02 & 43.2 & 3.6 & KIV \\
\hline 990131171713 & 46.9 & 42.9 & 4 & KIV \\
\hline 990131191804 & 46.49 & 43.01 & 4 & KIV \\
\hline 990201034347 & 46.52 & 43.02 & 3.9 & KIV \\
\hline 990201064942 & 50.71 & 40.5 & 3.9 & KIV \\
\hline 990201142552 & 47.61 & 43.34 & 5.2 & AKS AMB ARU KAP PAR VNN \\
\hline 990201142554 & 47.21 & 43.36 & 4.2 & KIV \\
\hline 990201235752 & 46.97 & 43.02 & 3.8 & KIV \\
\hline 990202045619 & 46.58 & 43.07 & 4 & KIV \\
\hline 990202055431 & 47 & 42.96 & 4 & KIV \\
\hline 990202070345 & 47.08 & 43 & 4 & KIV \\
\hline 990203142818 & 46.8 & 43.05 & 4 & KIV \\
\hline 990205082902 & 47.02 & 42.98 & 4 & KIV \\
\hline 990205165839 & 47.03 & 43.04 & 4 & KIV \\
\hline 990205214504 & 46.86 & 42.89 & 4 & KIV \\
\hline 990206100210 & 47.07 & 43.05 & 4 & KIV \\
\hline 990207131858 & 48.41 & 42.8 & 3.9 & KIV \\
\hline 990208112032 & 43.6 & 38.78 & 3.4 & KIV \\
\hline 990208202208 & 43.02 & 38.55 & 3.1 & KIV \\
\hline 990210144800 & 40.47 & 39.76 & 3.6 & KIV \\
\hline 990211041451 & 47.08 & 43.03 & 4 & KIV \\
\hline 990211052106 & 47.02 & 43.22 & 4 & KIV \\
\hline 990214222732 & 44.98 & 38.89 & 3.5 & KAP \\
\hline 990218220046 & 40.52 & 38.76 & 3.1 & GNI KIV \\
\hline 990219180001 & 44.74 & 38.28 & 4.3 & AKS \\
\hline 990219180015 & 44.51 & 38.81 & 4.3 & GNI KIV \\
\hline 990221031830 & 42.06 & 39.89 & 3.6 & AKS \\
\hline 990221031833 & 42.28 & 40.56 & 3.7 & GNI KIV \\
\hline 990221181436 & 46.91 & 43.26 & 5.1 & GNI KIV \\
\hline 990221203200 & 48.24 & 43.35 & 4.1 & GNI KIV \\
\hline 990222070850 & 47.05 & 42.83 & 4.1 & GNI KIV \\
\hline 990222075222 & 46.71 & 43.12 & 3.6 & GNI KIV \\
\hline 990222080031 & 47.07 & 43.11 & 4 & GNI KIV \\
\hline 990222105727 & 46.79 & 43.09 & 4 & GNI KIV \\
\hline 990222133306 & 49.27 & 42.85 & 5.4 & AKS KAP PAR VNN \\
\hline 990222133319 & 47.13 & 43.28 & 5.1 & GNI KIV \\
\hline 990223015408 & 46.98 & 43.02 & 4 & GNI KIV \\
\hline 990223191800 & 46.92 & 43.02 & 4.4 & GNI KIV \\
\hline 990223200906 & 46.65 & 43.07 & 4 & GNI KIV \\
\hline 990223222516 & 47 & 43.19 & 4.3 & GNI KIV \\
\hline 990224054933 & 46.92 & 43.12 & 4.1 & GNI KIV \\
\hline 990224130955 & 46.44 & 43.17 & 4 & GNI KIV \\
\hline 990224152309 & 45.71 & 42.92 & 4 & GNI KIV \\
\hline 990225185724 & 47.1 & 43.1 & 4 & GNI KIV \\
\hline 990301104639 & 40.81 & 39.2 & 4.2 & GNI KIV \\
\hline 990301111347 & 40.46 & 39.31 & 3.1 & GNI KIV \\
\hline 990301145352 & 41.24 & 38.14 & 4 & GNI KIV \\
\hline 990301175634 & 41.14 & 38.18 & 3.8 & GNI KIV \\
\hline 990305040654 & -12345 & -12345 & 0 & SPIK \\
\hline 990307055448 & 46.99 & 43.25 & 4 & GNI KIV \\
\hline 990309094155 & 44.94 & 39.37 & 3.5 & AKS \\
\hline 990309094204 & 44.93 & 39.13 & 4.2 & KIV \\
\hline
\end{tabular}




\begin{tabular}{|c|c|c|c|c|}
\hline 990313202218 & 46.88 & 43.11 & 4.2 & GNI KIV \\
\hline 990313211456 & 46.92 & 43.16 & 4 & GNI KIV \\
\hline 990314212031 & 47.05 & 43.05 & 4 & GNI KIV \\
\hline 990316003024 & 43.99 & 38.06 & 3.9 & PAR \\
\hline 990317015057 & 47.09 & 40.42 & 3.3 & GNI KIV \\
\hline 990317063030 & 46.79 & 42.97 & 4 & GNI KIV \\
\hline 990318124742 & 46.91 & 43.15 & 4 & GNI KIV \\
\hline 990320174945 & 47.04 & 43.18 & 4.4 & GNI KIV \\
\hline 990321093121 & 47.03 & 42.94 & 4 & GNI KIV \\
\hline 990323210658 & 46.76 & 44.12 & 4 & GNI KIV \\
\hline 990326064117 & 47 & 43.31 & 4 & GNI KIV \\
\hline 990329123039 & 47.53 & 42.14 & 4 & GNI KIV \\
\hline 990402041846 & -12345 & -12345 & 0 & SPIK \\
\hline 990402042652 & -12345 & -12345 & 0 & SPIK STPV \\
\hline 990403203204 & 45.25 & 42.17 & 4 & AKS \\
\hline 990403203206 & 44.98 & 42.9 & 3.4 & KIV \\
\hline 990405174547 & 42.19 & 39.72 & 4.1 & ARU \\
\hline 990405174553 & 42.28 & 39.59 & 3.8 & GNI KIV \\
\hline 990406000029 & -12345 & -12345 & 5.4 & DOG ELZ ELZ ELZ MLT \\
\hline 990407090912 & 48.19 & 42.31 & 4 & GNI KIV \\
\hline 990407124226 & 47.21 & 43.1 & 4 & GNI KIV \\
\hline 990407191900 & 46.97 & 42.91 & 4 & GNI KIV \\
\hline 990410022351 & -12345 & -12345 & 0 & SPIK \\
\hline 990410102229 & 46.88 & 43.01 & 4 & GNI KIV \\
\hline 990411233834 & 46.8 & 43.04 & 4 & GNI KIV \\
\hline 990413234454 & 47.07 & 43.22 & 3.9 & GNI KIV \\
\hline 990413235538 & 47.19 & 43.25 & 4 & GNI KIV \\
\hline 990414023115 & 46.91 & 43.04 & 4 & GNI KIV \\
\hline 990414032135 & 47.65 & 43.27 & 4 & GNI KIV \\
\hline 990414115610 & 41.57 & 39.19 & 3.2 & GNI KIV \\
\hline 990414161225 & 47.12 & 43.22 & 4 & GNI KIV \\
\hline 990415041201 & 46.28 & 41.88 & 4 & GNI KIV \\
\hline 990415111603 & 46.84 & 44 & 5.3 & AKS AMB PAR VNN \\
\hline 990415111612 & 47.08 & 43.32 & 4.1 & GNI KIV \\
\hline 990415112349 & 46.98 & 42.92 & 4 & GNI KIV \\
\hline 990415200127 & 46.98 & 43.24 & 4 & GNI KIV \\
\hline 990416135706 & 43.37 & 38.58 & 3.5 & GNI KIV \\
\hline 990416202145 & 44.88 & 38.53 & 3.5 & VNN \\
\hline 990417222832 & 46.93 & 43 & 4 & GNI KIV \\
\hline 990418102718 & 46.88 & 42.93 & 4 & GNI KIV \\
\hline 990418220512 & 40.18 & 39.31 & 3.4 & GNI KIV \\
\hline 990421002109 & 44.05 & 41.17 & 4.2 & GNI KIV \\
\hline 990424024119 & 45.92 & 41.91 & 4 & GNI KIV \\
\hline 990425124406 & 41.06 & 38.05 & 3.4 & GNI KIV \\
\hline 990425132841 & 41.04 & 38.11 & 3.3 & GNI KIV \\
\hline 990426210012 & 47.83 & 41.4 & 4 & GNI KIV \\
\hline 990502015353 & 45.94 & 43.13 & 4 & GNI KIV \\
\hline 990502062552 & 44.07 & 38.92 & 3.6 & AKS \\
\hline 990503115702 & 43.42 & 39.02 & 4.1 & GNI KIV \\
\hline 990503181522 & 46.68 & 42.76 & 4 & GNI KIV \\
\hline 990505193126 & 46.92 & 43.09 & 4 & GNI KIV \\
\hline 990505193558 & 46.9 & 43.04 & 4 & GNI KIV \\
\hline
\end{tabular}




\begin{tabular}{|c|c|c|c|c|}
\hline 990509094219 & 46.08 & 43.07 & 4 & GNI KIV \\
\hline 990509152109 & 41.18 & 38.96 & 3.5 & GNI KIV \\
\hline 990522141921 & 42.86 & 42.56 & 4.6 & KIV \\
\hline 990522141924 & 42.7 & 42.7 & 4.2 & ARU HAR \\
\hline 990524100425 & 44.39 & 40.95 & 4 & KIV \\
\hline 990525005243 & 46.84 & 42.96 & 4 & KIV \\
\hline 990525094645 & 50.26 & 40.51 & 4 & KIV \\
\hline 990528185911 & 44.98 & 43.08 & 4 & GNI KIV \\
\hline 990529222026 & 47.81 & 42.4 & 4 & GNI KIV \\
\hline 990530185932 & 46.4 & 43.06 & 4 & GNI KIV \\
\hline 990531083503 & 42.49 & 40.44 & 4.2 & GNI KIV \\
\hline 990602080843 & 47.04 & 43.13 & 4 & GNI KIV \\
\hline 990603000212 & 45.71 & 40.86 & 4 & GNI KIV \\
\hline 990603202019 & 44.13 & 42.81 & 4 & GNI KIV \\
\hline 990603215233 & 43.36 & 42.41 & 4 & GNI KIV \\
\hline 990604091242 & 47.63 & 40.48 & 5 & AKS ARU NPP PAR VRN \\
\hline 990604091251 & 47.46 & 40.76 & 5.5 & GNI KIV \\
\hline 990604104918 & 47.72 & 40.67 & 12345 & GNI KIV \\
\hline 990606113435 & 47.05 & 43.28 & 4.1 & GNI KIV \\
\hline 990610171954 & 46.85 & 43.05 & 4 & GNI KIV \\
\hline 990610172749 & 46.97 & 43 & 4 & GNI KIV \\
\hline 990616091202 & 47.63 & 42.16 & 4 & GNI KIV \\
\hline 990617181745 & 46.19 & 43.01 & 4 & GNI KIV \\
\hline 990620063042 & 40.22 & 38.7 & 3.4 & GNI KIV \\
\hline 990622072751 & 40.39 & 38.94 & 3.3 & GNI KIV \\
\hline 990624225744 & 46.37 & 43.48 & 4.3 & GNI KIV \\
\hline 990625142622 & 47 & 43.05 & 4 & GNI KIV \\
\hline 990626101839 & 42.92 & 43.17 & 4 & GNI KIV \\
\hline 990626102742 & 46.13 & 43.22 & 4.4 & GNI KIV \\
\hline 990630170657 & 46.58 & 41.86 & 4 & GNI KIV \\
\hline 990705185626 & 47 & 43.1 & 4 & GNI KIV \\
\hline 990706004646 & 46.2 & 42.66 & 4 & GNI KIV \\
\hline 990706205207 & 49.4 & 41.55 & 4 & GNI KIV \\
\hline 990707002322 & 46.93 & 43.13 & 4 & GNI KIV \\
\hline 990707134849 & 48.36 & 41.88 & 4 & GNI KIV \\
\hline 990707174312 & 48.22 & 41.1 & 4 & GNI KIV \\
\hline 990711113101 & 43.49 & 38.54 & 3.4 & GNI KIV \\
\hline 990712212139 & 40.22 & 39.16 & 4 & GNI KIV \\
\hline 990714161544 & 47.19 & 42.99 & 4 & GNI KIV \\
\hline 990714162239 & 47.15 & 43.05 & 4 & GNI KIV \\
\hline 990714174329 & 47.41 & 41.99 & 4 & GNI KIV \\
\hline 990714195757 & 47.5 & 41.97 & 4 & GNI KIV \\
\hline 990717103709 & 40.04 & 38.35 & 3.8 & GNI KIV \\
\hline 990717123844 & 47.46 & 42.13 & 4 & GNI KIV \\
\hline 990719072522 & 47.62 & 42.21 & 4 & GNI KIV \\
\hline 990719151343 & 47.34 & 42.02 & 4 & GNI KIV \\
\hline 990719160645 & 47.37 & 41.9 & 4 & GNI KIV \\
\hline 990720042242 & 41.34 & 39.9 & 4.6 & GNI KIV \\
\hline 990720082954 & 47.37 & 42.1 & 4 & GNI KIV \\
\hline 990721095649 & 46.2 & 43.22 & 4 & GNI KIV \\
\hline 990722155431 & 47.82 & 41.83 & 4 & GNI KIV \\
\hline 990723202615 & 47.09 & 43 & 4 & GNI KIV \\
\hline
\end{tabular}




\begin{tabular}{|c|c|c|c|c|}
\hline 990725013753 & 46.98 & 43.13 & 4 & GNI KIV \\
\hline 990725092628 & 46.91 & 43.19 & 4 & GNI KIV \\
\hline 990727235632 & 46.59 & 43.01 & 4 & GNI KIV \\
\hline 990729205014 & 47.42 & 41.99 & 4 & GNI KIV \\
\hline 990802090930 & 46.92 & 42.85 & 4 & GNI KIV \\
\hline 990802234404 & 46.88 & 43.04 & 4 & GNI KIV \\
\hline 990803005107 & 46.95 & 42.98 & 4 & GNI KIV \\
\hline 990803225330 & 46.72 & 43.05 & 4 & GNI KIV \\
\hline 990806000307 & 49.46 & 41.86 & 4 & KIV \\
\hline 990808184822 & 46.39 & 41.74 & 4 & GNI KIV \\
\hline 990809103710 & 47.9 & 42 & 4 & GNI KIV \\
\hline 990809115708 & 48.1 & 42.25 & 4 & GNI KIV \\
\hline 990809150742 & 49.78 & 41.76 & 4 & GNI KIV \\
\hline 990810121824 & 47.07 & 43.16 & 4 & GNI KIV \\
\hline 990810182127 & 46.96 & 43.06 & 12345 & GNI KIV \\
\hline 990811002623 & 46.77 & 42.93 & 4 & GNI KIV \\
\hline 990811044713 & 46.35 & 42.78 & 4 & GNI KIV \\
\hline 990814125911 & 47.11 & 43.07 & 4 & GNI KIV \\
\hline 990815202158 & 46.27 & 42.49 & 4 & GNI KIV \\
\hline 990819043310 & 47.55 & 38.95 & 4.8 & AKS \\
\hline 990819043319 & 46.42 & 38.42 & 3.9 & GNI KIV \\
\hline 990821183118 & 42.25 & 39.97 & 3.5 & HAR PAR \\
\hline 990821184151 & 40.38 & 39.26 & 4 & GNI KIV \\
\hline 990822111258 & 40.17 & 39.28 & 4 & GNI KIV \\
\hline 990822121609 & 40.41 & 39.12 & 3.9 & GNI KIV \\
\hline 990823153439 & 40.15 & 38.92 & 3.2 & GNI KIV \\
\hline 990823194635 & 40.42 & 39.32 & 3.5 & GNI KIV \\
\hline 990824063242 & 40.28 & 39.28 & 3.3 & GNI KIV \\
\hline 990825124853 & 49.25 & 41.7 & 4 & GNI KIV \\
\hline 990826221316 & 40.23 & 39.46 & 3.9 & GNI KIV \\
\hline 990827074027 & 47.34 & 43.04 & 4 & GNI KIV \\
\hline 990903153701 & 47.01 & 43.16 & 4 & GNI KIV \\
\hline 990904015759 & 46.8 & 42.93 & 4 & GNI KIV \\
\hline 990904204548 & 50.84 & 40.26 & 4.2 & GNI KIV \\
\hline 990905083205 & 40.51 & 39.37 & 3.6 & GNI KIV \\
\hline 990906215324 & 45.28 & 41.72 & 4 & GNI KIV \\
\hline 990907203546 & 47.2 & 42.93 & 4 & GNI KIV \\
\hline 990908023005 & 40.46 & 39.35 & 3.2 & GNI KIV \\
\hline 990909124833 & 40.03 & 39.41 & 3 & GNI KIV \\
\hline 990909125507 & 40.29 & 39.7 & 3.2 & GNI KIV \\
\hline 990910083705 & 40.32 & 39.28 & 2.9 & GNI KIV \\
\hline 990913092352 & 46.95 & 43.12 & 4 & GNI KIV \\
\hline 990919135553 & 47.86 & 42.38 & 4 & KIV \\
\hline 990919164658 & 46.93 & 43.21 & 12345 & GNI KIV \\
\hline 990919164700 & 46.88 & 43.15 & 4.8 & GNI KIV \\
\hline 990929115449 & 46.14 & 41.97 & 4.2 & GNI KIV \\
\hline 990930034042 & 40.91 & 39.66 & 3.4 & GNI KIV \\
\hline 991002014014 & 45.81 & 42.88 & 4.1 & GNI KIV \\
\hline 991002070656 & 47.47 & 42.22 & 3.7 & GNI KIV \\
\hline 991002202459 & 48.04 & 42.32 & 3.9 & GNI KIV \\
\hline 991003131917 & 48.66 & 38.92 & 4.7 & GNI KIV \\
\hline 991003131934 & 48 & 40.83 & 4 & VNN \\
\hline
\end{tabular}




\begin{tabular}{|c|c|c|c|c|}
\hline 991007232345 & 43.9 & 42.77 & 4.3 & GNI KIV \\
\hline 991010055913 & 46.55 & 40.83 & 4.1 & GNI KIV \\
\hline 991012154115 & 48.3 & 39.87 & 4.6 & ARU NAR \\
\hline 991012154116 & 48.32 & 39.14 & 4.7 & GNI KIV \\
\hline 991013100039 & 40.28 & 40.16 & 3.7 & GNI KIV \\
\hline 991015175301 & 47.01 & 43.15 & 3.6 & GNI KIV \\
\hline 991019181843 & 42.69 & 44.95 & 4.4 & GNI KIV \\
\hline 991021180350 & 46.1 & 42.12 & 3.7 & GNI KIV \\
\hline 991022154523 & 40.02 & 38.82 & 3.3 & GNI KIV \\
\hline 991023180904 & 46.94 & 42.82 & 3.5 & EZRM GNI KIV \\
\hline 991024173228 & 40.22 & 39.11 & 3.3 & DGRL EZRM GNI HRSN KIV \\
\hline 991025014335 & 40.4 & 39.33 & 3.4 & DGRL EZRM GNI HRSN KIV \\
\hline 991025100740 & 40.14 & 39.05 & 3.6 & DGRL EZRM GNI HRSN KIV \\
\hline 991026210038 & 46.92 & 43.15 & 4.2 & DGRL EZRM GNI HINS HRSN KARS KIV KOTK KRLV \\
\hline 991031162151 & 41.48 & 38.6 & 3.6 & BYBT CMCY DGRL DGSU EZRM GNI HAMR HINS HRSN KARS KIV KOTK KRLV \\
\hline 991031200601 & 48.33 & 42.59 & 3.9 & BTLS BYBT CMCY DGRL DGSU EZRM GNI HAMR HINS HRSN KARS KIV KOTK KRLV \\
\hline 991123062737 & 43.07 & 39.98 & 4.4 & AHLT ARU BNGL BYBT BYKN CMCY DGSU EZRM GNI HRSN KARS KIV KRLV LER SILN \\
\hline 991127072647 & 45.67 & 38.77 & 3.5 & ARU \\
\hline 991128054409 & 40.7 & 38.86 & 3.6 & AHLT BNGL BYBT BYKN CMCY DGSU EZRM GNI HAMR HINS HRSN KARS KIV KOTK KRLV SILN \\
\hline 991203170654 & 42.36 & 40.41 & 5.3 & AHLT BNGL BYBT BYKN CMCY DGSU EZRM GNI HAMR HINS HRSN KARS KIV KOTK KRLV MUSH SILN \\
\hline 991203183230 & 42.25 & 40.08 & 3.5 & ARU LER \\
\hline 991203200654 & 42.26 & 40.35 & 4 & AHLT BNGL BYBT BYKN CMCY DGSU EZRM GNI HAMR HINS HRSN KARS KIV KOTK KRLV MUSH SILN \\
\hline 991203200752 & 42.17 & 40.2 & 4.1 & AKS \\
\hline 991203204023 & 42.17 & 40.2 & 4.1 & AKS \\
\hline 991203213310 & 42.44 & 40.43 & 3.8 & AHLT BNGL BYBT BYKN CMCY DGSU EZRM GNI HAMR HINS HRSN KARS KIV KOTK KRLV MUSH SILN \\
\hline 991204002001 & 42.32 & 40.33 & 3.7 & AKS \\
\hline 991205024851 & 41.93 & 39.3 & 3.2 & AHLT BNGL BYBT BYKN CMCY DGSU EZRM GNI HAMR HINS HRSN KARS KIV KOTK KRLV MUSH SILN \\
\hline 991205105215 & 47 & 43.08 & 4.2 & AHLT BNGL BYBT BYKN CMCY DGSU EZRM GNI HAMR HINS HRSN KARS KIV KOTK KRLV MUSH SILN \\
\hline 991205221436 & 42.27 & 40.43 & 3.9 & AMB KAM \\
\hline 991205221437 & 42.54 & 40.58 & $\begin{array}{r}4.6 \\
-\end{array}$ & AHLT BNGL BYBT BYKN CMCY DGSU EZRM GNI HAMR HINS HRSN KARS KIV KOTK KRLV MUSH SILN \\
\hline 991207130909 & 42.24 & 40.33 & 12345 & AHLT BNGL BYBT BYKN CMCY DGSU EZRM GNI HAMR HINS HRSN KARS KIV KOTK KRLV MUSH SILN \\
\hline 991221001228 & 42.42 & 40.37 & 3.9 & AHLT BNGL BYBT BYKN CMCY DGSU EZRM GNI HAMR HINS HRSN KARS KIV KOTK KRLV MUSH SILN \\
\hline 991222065645 & 43.32 & 43.71 & 3.5 & AHLT BNGL BYBT BYKN CMCY DGSU EZRM GNI HAMR HINS HRSN KARS KIV KOTK KRLV MUSH SILN \\
\hline 991224052439 & 46.45 & 42.43 & 4 & AHLT BNGL BYBT BYKN CMCY DGSU EZRM GNI HAMR HINS HRSN KARS KIV KOTK KRLV MUSH SILN \\
\hline 991224235509 & 42.11 & 39.04 & 3.6 & AHLT BNGL BYBT BYKN CMCY DGSU EZRM GNI HAMR HINS HRSN KARS KIV KOTK KRLV MUSH SILN \\
\hline 991225035301 & 48.28 & 40.86 & 3.9 & AHLT BNGL BYBT BYKN CMCY DGSU EZRM GNI HAMR HINS HRSN KARS KIV KOTK KRLV MUSH SILN \\
\hline
\end{tabular}

\section{*-12345 denotes that the data are not in the SAC header file}

